JOURNAL OF THE

AMERICAN MATHEMATICAL SOCIETY

Volume 23, Number 4, October 2010, Pages 909-966

S 0894-0347(10)00669-7

Article electronically published on April 1, 2010

\title{
INTEGRAL TRANSFORMS AND DRINFELD CENTERS IN DERIVED ALGEBRAIC GEOMETRY
}

\author{
DAVID BEN-ZVI, JOHN FRANCIS, AND DAVID NADLER
}

Compact objects are as necessary to this subject as air to breathe.

R.W. Thomason to A. Neeman,

\section{Contents}

1. Introduction

1.1. Perfect stacks

1.2. Tensors and functors

1.3. Centers and traces

1.4. Hecke categories

1.5. Topological field theory

2. Preliminaries

2.1. $\infty$-categories

2.2. Monoidal $\infty$-categories

2.3. Derived algebraic geometry

2.4. Derived loop spaces

2.5. $\mathcal{E}_{n}$-structures

3. Perfect stacks

3.1. Definition of perfect stacks

3.2. Base change and the projection formula 932

3.3. Constructions of perfect stacks $\quad 934$

4. Tensor products and integral transforms 939

4.1. Tensor products of $\infty$-categories $\quad 940$

4.2. Sheaves on fiber products 944

4.3. General base stacks 948

5. Applications $\quad 951$

5.1. Centers and traces 951

5.2. Centers of convolution categories 954

5.3. Higher centers 957

6. Epilogue: Topological field theory $\quad 960$

6.1. Topological field theory from perfect stacks 960

6.2. Deligne-Kontsevich conjectures for derived centers 962

Received by the editors October 23, 2008 and, in revised form, March 4, 2010.

2010 Mathematics Subject Classification. Primary 14-XX; Secondary 55-XX.

(c) 2010 American Mathematical Society Reverts to public domain 28 years from publication 
Acknowledgements $\quad 963$

References 963

\section{INTRODUCTION}

This paper is devoted to the study of natural algebraic operations on derived categories arising in algebraic geometry. Our main goal is to identify the category of sheaves on a fiber product with two algebraic constructions: on the one hand, the tensor product of the categories of sheaves on the factors, and on the other hand, the category of linear functors between the categories of sheaves on the factors (thereby realizing functors as integral transforms). Among the varied applications of our main results are the calculation of Drinfeld centers (and higher centers) of monoidal categories of sheaves and the construction of topological field theories.

For such questions about the linear algebra of derived categories to be tractable, we work in an enriched setting, where we replace triangulated categories with a more refined homotopy theory of categories. Over a ring $k$ of characteristic zero, one solution is provided by pretriangulated differential graded categories (see for example the survey [Ke], as well as [D, To1]). The theory of stable $\infty$-categories as presented in [L2, L3] (building on the quasi-categories of [Jo]) provides a more general solution. We adopt this formalism thanks to the comprehensive foundations available in [L2, L3, L4, L5. In Section 2 below, we provide a recapitulation of this theory sufficient for the arguments of this paper. The reader interested in characteristic zero applications may consistently substitute differential graded categories for $\infty$-categories throughout.

To any scheme or stack $X$, we can assign a stable $\infty$-category $\mathrm{QC}(X)$ which has the usual unbounded quasi-coherent derived category $D_{q c}(X)$ as its homotopy category. The tensor product of sheaves provides $\mathrm{QC}(X)$ with the structure of a symmetric monoidal stable $\infty$-category. Our aim is to calculate the $\infty$-categories built out of $\mathrm{QC}(X)$ by taking tensor products, linear functors, and more intricate algebraic constructions in terms of the geometry of $X$.

More generally, instead of an ordinary scheme or stack, our starting point will be a derived stack in the sense of derived algebraic geometry as developed in L1, L3, L4, L5, ToVe1, ToVe2, (see also To2 for a concise survey, and Section 2 below for a brief primer). Recall that stacks and higher stacks arise naturally from performing quotients (and more complicated colimits) on schemes. Thus we correct the notion of forming quotients by passing to stacks. Likewise, derived stacks arise naturally from taking fiber products (and more complicated limits) on schemes and stacks. Thus we correct the notion of imposing an equation by passing to derived stacks. It is worth mentioning that they also arise naturally in more general contexts for doing algebraic geometry that are important for stable homotopy theory.

Recall that the functor of points of a scheme assigns a set to any commutative ring. Roughly speaking, a derived stack assigns a topological space to any derived commutative ring (for precise definitions, see Section 2 below). There are many variations on what one could mean by a commutative derived ring as discussed in Section 2.3. For example, in characteristic zero, one can work with (connective, or homological) commutative differential graded algebras. A more general context, which we typically adopt, is the formalism of connective $\mathcal{E}_{\infty}$-ring spectra. Since the 
techniques of this paper apply in all of the usual contexts, we will often not specify our context further and use the term commutative derived ring as a catch-all for any of them.

To any derived stack $X$, we can assign a stable $\infty$-category $\mathrm{QC}(X)$ extending the definition for ordinary schemes and stacks. In particular, for an affine derived scheme $X=\operatorname{Spec} R$, by definition $\mathrm{QC}(X)$ is the $\infty$-category of $R$-modules $\operatorname{Mod}_{R}$ whose homotopy category is the usual unbounded derived category of $R$-modules. The tensor product provides $\mathrm{QC}(X)$ with the structure of a symmetric monoidal stable $\infty$-category.

Our main technical results are two algebraic identifications of the $\infty$-category of quasi-coherent sheaves on a fiber product: first, we identify it with the tensor product of the $\infty$-categories of sheaves on the factors over the $\infty$-category of sheaves on the base; second, we identify it with the $\infty$-category of functors between the $\infty$ categories of sheaves on the factors that are linear over the $\infty$-category of sheaves on the base (thereby realizing functors as integral transforms). Our results hold for a broad class of stacks, called perfect stacks, which we introduce in the next section immediately below.

Our main applications are the calculation of the Drinfeld centers (and higher $\mathcal{E}_{n}$-centers) of $\infty$-categories of sheaves and functors. For example, we identify the Drinfeld center of the quasi-coherent affine Hecke category with sheaves on the moduli of local systems on a torus. We also explain how all of our results fit into the framework of 3-dimensional topological field theory (specifically of RozanksyWitten type). In particular, we verify categorified analogues of the Deligne and Kontsevich conjectures on the $\mathcal{E}_{n}$-structure of Hochschild cohomology.

1.1. Perfect stacks. For an arbitrary derived stack $X$, the $\infty$-category $\mathrm{QC}(X)$ is difficult to control algebraically. For example, it may contain large objects that are impossible to construct in terms of concrete, locally-finite objects.

To get a handle on $\mathrm{QC}(X)$, we need to know that it has a small $\infty$-subcategory $\mathrm{QC}(X)^{\circ}$ of "generators" which are "finite" in an appropriate sense. There are two common notions of when a small subcategory $\mathcal{C}^{\circ}$ generates a category $\mathcal{C}$, and they have natural $\infty$-analogues. On the one hand, we could ask that $\mathcal{C}$ be the inductive limit or ind-category Ind $\mathcal{C}^{\circ}$ (i.e., that $\mathcal{C}$ is freely generated from $\mathcal{C}^{\circ}$ by taking inductive limits), and on the other hand, we could ask that in $\mathcal{C}$ the right orthogonal of $\mathcal{C}^{\circ}$ vanishes. When $\mathcal{C}$ is $\mathrm{QC}(X)$, there are three common notions of which objects may be considered finite: perfect objects, dualizable objects, and compact objects, which refer respectively to the geometry, monoidal structure, and categorical structure of $\mathrm{QC}(X)$. We review all of these notions and the relations between them in Section 3.1 (in particular, perfect and dualizable objects always coincide).

To have a tractable and broadly applicable class of derived stacks, we introduce the notion of a perfect stack. By definition, an object $M$ of $\mathrm{QC}(X)$ is a perfect complex if locally for any affine $U \rightarrow X$ its restriction to $U$ is a perfect module (finite complex of projective modules). Equivalently, $M$ is dualizable with respect to the monoidal structure on $\mathrm{QC}(X)$. A derived stack $X$ is said to be perfect if it has affine diagonal and the $\infty$-category $\mathrm{QC}(X)$ is the inductive limit

$$
\mathrm{QC}(X) \simeq \operatorname{Ind} \operatorname{Perf}(X)
$$


of the full $\infty$-subcategory $\operatorname{Perf}(X)$ of perfect complexes. A morphism $X \rightarrow Y$ is said to be perfect if its fibers $X \times_{Y} U$ over affines $U \rightarrow Y$ are perfect.

On a perfect stack $X$, compact objects of $\mathrm{QC}(X)$ are the same thing as perfect complexes (which in turn are always the same thing as dualizable objects). In fact, we have the following alternative formulation: a derived stack $X$ is perfect if it has affine diagonal, $\mathrm{QC}(X)$ is compactly generated (that is, there is no right orthogonal to the compact objects), and compact objects and dualizable objects coincide.

Remark 1.1. The notion of compactly generated categories is a standard one in homotopy theory, especially in conjunction with themes such as Brown representability and Bousfield localization. Schwede and Shipley SSh prove in great generality that compactly generated categories can be expressed as categories of modules.

In algebraic geometry, the importance of the interplay between compact and perfect objects was originally recognized and put to great use by Thomason [TT. These ideas were combined with homotopical techniques by Bökstedt and Neeman BoN] and further developed and enhanced by Neeman [N1, N2 and many others Ke, BV, To1. The key property of derived categories of quasi-coherent sheaves on quasi-compact, separated schemes identified in these papers is that on the one hand, they are compactly generated, and on the other hand, their compact and perfect objects coincide. Such categories appear as unital algebraic stable homotopy categories in the general axiomatic framework developed by Hovey, Palmieri and Strickland HPS. This combination of properties underlies the definition of a perfect stack.

In Section 3.2, we establish base change and the projection formula for perfect morphisms following arguments in Lurie's thesis [L1] (in fact, the arguments here only use that the structure sheaf is relatively compact).

In Section 3.3. we show that the class of perfect stacks is very broad. We show that the following are all perfect stacks:

(1) Quasi-compact derived schemes with affine diagonal (following arguments of [N2]).

(2) The total space of a quasi-projective morphism over a perfect base.

(3) In characteristic zero, the quotient $X / G$ of a quasi-projective derived scheme $X$ by a linear action of an affine group $G$.

(4) The quotient $X / G$ of a perfect stack $X$ by a finite affine group scheme in "good" characteristics for $G$.

(5) The mapping stack $X^{\Sigma}=\operatorname{Map}(\Sigma, X)$, for a perfect stack $X$ and a finite simplicial set $\Sigma$.

(6) Fiber products of perfect stacks.

We also show that any morphism $X \rightarrow Y$ between perfect stacks is itself perfect.

Though the above examples show that perfect stacks cover a broad array of spaces of interest, it is worth pointing out that there are many commonly arising derived stacks that are imperfect. Since categories of quasi-coherent sheaves are usually compactly generated, the typical reason for $X$ to be imperfect is that the structure sheaf $\mathcal{O}_{X}$ (which is always dualizable) fails to be compact (or equivalently, the global sections functor fails to preserve colimits). This can occur if the cohomology $\Gamma\left(X, \mathcal{O}_{X}\right)$ is too big such as for (1) the classifying space of a finite group in modular characteristic (for the simplest example, one can take $B \mathbb{Z} / 2 \mathbb{Z}$ when 2 is not invertible), (2) the classifying space of a topological group such as $S^{1}$, or (3) an 
ind-scheme such as the formal disc Spf $k[[t]]$. In the opposite direction, categories of $\mathcal{D}$-modules on smooth schemes (which can be considered as quasi-coherent sheaves on the corresponding de Rham stacks) have compact unit, but also many compact objects (such as $\mathcal{D}$ itself) which are not dualizable.

1.2. Tensors and functors. For $X, X^{\prime}$ ordinary schemes over a ring $k$, a theorem of Toën [To1] identifies the dg category of $k$-linear continuous (that is, colimitpreserving) functors with the dg category of integral kernels

$$
\operatorname{Fun}_{k}\left(\mathrm{QC}(X), \mathrm{QC}\left(X^{\prime}\right)\right) \simeq \mathrm{QC}\left(X \times_{k} X^{\prime}\right) .
$$

For dg categories of perfect (equivalently, bounded coherent) complexes on smooth projective varieties, an analogous result was proved by Bondal, Larsen and Lunts BLL as well as by Toën [To1] (generalizing Orlov's theorem O] characterizing equivalences as Fourier-Mukai transforms).

We've collected our main technical results in the following generalization. In the statement, the tensors and functors of $\infty$-categories of quasi-coherent sheaves are calculated in the symmetric monoidal $\infty$-category $\mathcal{P} r^{\mathrm{L}}$ of presentable $\infty$-categories with morphisms being left adjoints (as developed in [L4, 4] and [L5, 5]; see Section 2 for a precise summary). The tensors and functors of $\infty$-categories of perfect complexes are calculated in the symmetric monoidal $\infty$-category st of $k$-linear idempotent complete stable small $\infty$-categories (as developed in Section 4.1 below).

Theorem 1.2. (1) For $X \rightarrow Y \leftarrow X^{\prime}$ maps of perfect stacks, there is a canonical equivalence

$$
\mathrm{QC}\left(X \times_{Y} X^{\prime}\right) \simeq \mathrm{QC}(X) \otimes_{\mathrm{QC}(Y)} \mathrm{QC}\left(X^{\prime}\right)
$$

between the $\infty$-category of sheaves on the derived fiber product and the tensor product of the $\infty$-categories of sheaves on the factors.

There is also a canonical equivalence

$$
\operatorname{Perf}\left(X \times X^{\prime}\right) \simeq \operatorname{Perf}(X) \otimes \operatorname{Perf}\left(X^{\prime}\right)
$$

for $\infty$-categories of perfect complexes.

(2) For $X \rightarrow Y$ a perfect morphism to a derived stack $Y$ with affine diagonal, and $X^{\prime} \rightarrow Y$ arbitrary, there is a canonical equivalence

$$
\mathrm{QC}\left(X \times_{Y} X^{\prime}\right) \simeq \operatorname{Fun}_{\mathrm{QC}(Y)}\left(\mathrm{QC}(X), \mathrm{QC}\left(X^{\prime}\right)\right)
$$

between the $\infty$-category of sheaves on the derived fiber product and the $\infty$ category of colimit-preserving $\mathrm{QC}(Y)$-linear functors.

When $X$ is a smooth and proper perfect stack, there is also a canonical equivalence

$$
\operatorname{Perf}\left(X \times X^{\prime}\right) \simeq \operatorname{Fun}\left(\operatorname{Perf}(X), \operatorname{Perf}\left(X^{\prime}\right)\right)
$$

for $\infty$-categories of perfect complexes.

Our arguments in Section 4 first establish the pair of assertions about tensors, then deduce sufficient duality to conclude the pair of assertions about functors.

Remark 1.3. The hypothesis that our stacks are perfect appears to be essential, and we do not expect the above theorem to hold in significantly greater generality. Alternatively, in complete generality, one should rather replace the notion of tensor product. Jacob Lurie has described (in a private communication) a completed tensor product for stable $\infty$-categories equipped with $t$-structures, and such that 
passing to quasi-coherent sheaves takes fiber products of geometric stacks to the completed tensor product of $\infty$-categories.

1.3. Centers and traces. A basic operation on associative algebras is the calculation of their center. The derived version of the center of an associative algebra $A$ is the Hochschild cochain complex (or simply the Hochschild cohomology), which calculates the derived endomorphisms of $A$ as an $A$-bimodule. Another basic operation is the calculation of the universal trace (i.e., the universal target for a map out of $A$ coequalizing left and right multiplication). The derived version of the universal trace is the Hochschild chain complex (or the Hochschild homology), which calculates the derived tensor product of $A$ with itself as an $A$-bimodule.

In Section [5.1] we extend the notion of Hochschild homology and cohomology to associative (or $\mathcal{E}_{1}$-)algebra objects in arbitrary closed symmetric monoidal $\infty$ categories. (As with any structure in an $\infty$-category, an associative multiplication, or $\mathcal{E}_{1}$-structure, is a homotopy coherent notion.) In the case of chain complexes, we recover the usual Hochschild chain and cochain complexes. In the case of spectra, we recover topological Hochschild homology and cohomology.

Definition 1.4. Let $A$ be an associative algebra object in a closed symmetric monoidal $\infty$-category $\mathcal{S}$.

(1) The derived center or Hochschild cohomology $\mathcal{Z}(A)=\mathrm{HH}^{*}(A) \in \mathcal{S}$ is the endomorphism object $\mathcal{E} n d_{A \otimes A^{\text {op }}}(A)$ of $A$ as an $A$-bimodule.

(2) The derived trace or Hochschild homology $\mathcal{T r}(A)=\mathrm{HH}_{*}(A) \in \mathcal{S}$ is the pairing object $A \otimes_{A \otimes A^{\text {op }}} A$ of $A$ with itself as an $A$-bimodule.

We show in particular that $\operatorname{HH}^{*}(A)$ and $\operatorname{HH}_{*}(A)$ are calculated in this generality by a version of the usual Hochschild complexes, the cyclic bar construction.

We apply this definition in the following setting. We will take $\mathcal{S}$ to be the $\infty$ category $\mathcal{P} r^{\mathrm{L}}$ of presentable $\infty$-categories with morphisms being left adjoints. Then an associative algebra object in $\mathcal{P} r^{\mathrm{L}}$ is a monoidal presentable $\infty$-category $\mathcal{C}$. Thus we have the notion of its center (or Hochschild cohomology category) and trace (or Hochschild homology category)

$$
\mathcal{Z}(\mathcal{C})=\operatorname{Fun}_{\mathcal{C} \otimes \mathcal{C}^{\mathrm{op}}}(\mathcal{C}, \mathcal{C}), \quad \mathcal{T} r(\mathcal{C})=\mathcal{C} \otimes_{\mathcal{C} \otimes \mathcal{C}^{\mathrm{op}}} \mathcal{C} .
$$

These are again presentable $\infty$-categories, or in other words, objects of the $\infty$ category $\mathcal{P} r^{\mathrm{L}}$. The center $\mathcal{Z}(\mathcal{C})$ comes equipped with a universal central functor to $\mathcal{C}$, and the trace $\operatorname{Tr}(\mathcal{C})$ receives a universal trace functor from $\mathcal{C}$.

Remark 1.5. The above notion of center provides a derived version of the Drinfeld center of a monoidal category (as defined in [JS). To appreciate the difference, consider the abelian tensor category $R$ - mod of modules over a (discrete) commutative ring $R$. Its classical Drinfeld center is $R$ - mod again since there are no nontrivial $R$-linear braidings for $R$-modules. But as we will see below, the derived center of the $\infty$-category of $R$-modules is the $\infty$-category of modules over the Hochschild chain complex of $R$.

Remark 1.6. It is important not to confuse the center $\mathcal{Z}(\mathcal{C})$ of a monoidal $\infty$ category $\mathcal{C}$ with the endomorphisms of the identity functor of the underlying $\infty$ category. The former is again an $\infty$-category depending on the monoidal structure of $\mathcal{C}$, while the latter is an algebra with no relation to the monoidal structure of $\mathcal{C}$. For example if $\mathcal{C}=A-\bmod$ is modules over an associative $A_{\infty}$-algebra $A$, then the 
endomorphisms of the identity of $\mathcal{C}$ are calculated by the (topological) Hochschild cohomology of $A$, not of the $\infty$-category $\mathcal{C}$.

Now let us return to a geometric setting and consider a perfect stack $X$ and the presentable $\infty$-category $\mathrm{QC}(X)$. Since $\mathrm{QC}(X)$ is symmetric monoidal, it defines a commutative (or $\mathcal{E}_{\infty^{-}}$)algebra object in $\mathcal{P} r^{\mathrm{L}}$ and so, in particular, an associative algebra object.

To calculate the center and trace of $\mathrm{QC}(X)$, we introduce the loop space

$$
\mathcal{L} X=\operatorname{Map}\left(S^{1}, X\right) \simeq X \times_{X \times X} X,
$$

where the derived fiber product is along two copies of the diagonal map.

For example, when $X$ is an ordinary smooth scheme over a field of characteristic zero, the loop space $\mathcal{L} X$ is the total space $T_{X}[-1]=\operatorname{Spec} \operatorname{Sym} \Omega_{X}[1]$ of the shifted tangent bundle of $X$. When $X$ is the classifying space $B G$ of a group $G$, the loop space $\mathcal{L} B G$ is the adjoint quotient $G / G$.

In Section 5.1 as a corollary of our main technical results, we obtain the following.

Theorem 1.7. For a perfect stack $X$, there are canonical equivalences

$$
\mathcal{Z}(\mathrm{QC}(X)) \simeq \mathrm{QC}(\mathcal{L} X) \simeq \mathcal{T} r(\mathrm{QC}(X))
$$

between its center, trace, and the $\infty$-category of sheaves on its loop space.

Note that the theorem in particular identifies the Hochschild homology and cohomology objects associated to the monoidal $\infty$-category $\mathrm{QC}(X)$. While such an identification may initially appear surprising, it is a natural consequence of the self-duality of $\mathrm{QC}(X)$ over $\mathrm{QC}(X \times X)$, which in turn is a simple consequence of Theorem 1.2 .

Remark 1.8. The theorem is a direct generalization of a result of Hinich $[\mathrm{H}]$. He proves that for $X$ a Deligne-Mumford stack admitting an affine orbifold chart, the Drinfeld center of the (abelian) tensor category of quasi-coherent sheaves on $X$ is equivalent to the category of quasi-coherent sheaves on the inertia orbifold of $X$ (with braided monoidal structure coming from convolution). One can recover this from the above theorem by passing to the heart of the natural $t$-structures.

In Section 5.3 we also discuss a generalization of the theorem to $\mathcal{E}_{n}$-centers and traces when $n>1$. First, we introduce the notion of center and trace for an $\mathcal{E}_{n^{-}}$ algebra object in $\mathcal{P} r^{\mathrm{L}}$, for any $n$. Since $\mathrm{QC}(X)$ is an $\mathcal{E}_{\infty}$-algebra object in $\mathcal{P} r^{\mathrm{L}}$, it is also an $\mathcal{E}_{n}$-algebra object, for any $n$.

We show that the $\mathcal{E}_{n}$-center and trace of $\mathrm{QC}(X)$ are equivalent to the $\infty$-category of quasi-coherent sheaves on the derived mapping space

$$
X^{S^{n}}=\operatorname{Map}\left(S^{n}, X\right) \text {. }
$$

For example, when $X$ is an ordinary smooth scheme over a field of characteristic zero, the $n$-sphere space $X^{S^{n}}$ is the total space $T_{X}[-n]=\operatorname{Spec} \operatorname{Sym} \Omega_{X}[n]$ of the shifted tangent bundle of $X$. In particular, as we vary $n$, the $\mathcal{E}_{n}$-centers and traces of $\mathrm{QC}(X)$ differ from $\mathrm{QC}(X)$, though they all have $t$-structures with heart the abelian category of quasi-coherent sheaves on $X$.

When $X$ is the classifying space $B G$ of a group $G$, the $n$-sphere space $B G^{S^{n}}$ can be interpreted as the derived stack $\mathcal{L}_{o c}\left(S^{n}\right)$ of $G$-local systems on $S^{n}$. In particular, when $n=2$, the $\mathcal{E}_{2}$-center and trace of $\mathrm{QC}(B G)$ is the $\infty$-category 
$\mathrm{QC}\left(\mathcal{L}_{o c}\left(S^{2}\right)\right)$ which appears in the Geometric Langlands program (see, for example, the work of Bezrukavnikov and Finkelberg $\left[\mathrm{BeF}\right.$ ] who identify $\mathrm{QC}\left(\mathcal{L}_{o c_{G}}\left(S^{2}\right)\right)$ with the derived Satake (or spherical Hecke) category of arc-group equivariant constructible sheaves on the affine Grassmannian for the dual group).

1.4. Hecke categories. Let $X \rightarrow Y$ be a map of perfect stacks. Our main technical result gives an identification of (not necessarily symmetric) monoidal $\infty$ categories

$$
\mathrm{QC}\left(X \times_{Y} X\right) \simeq \operatorname{Fun}_{\mathrm{QC}(Y)}(\mathrm{QC}(X), \mathrm{QC}(X)),
$$

where the left hand side is equipped with convolution and the right hand side with the composition of functors. In other words, we have an identification of associative algebra objects in the $\infty$-category $\mathcal{P} r$ of presentable $\infty$-categories with morphisms the left adjoints.

In Section 5.2, we calculate the center of $\mathrm{QC}\left(X \times_{Y} X\right)$ in the following form. Recall that $\mathcal{L} Y$ denotes the derived loop space of $Y$.

Theorem 1.9. Suppose $p: X \rightarrow Y$ is a map of perfect stacks that satisfies descent. Then there is a canonical equivalence

$$
\mathcal{Z}\left(\mathrm{QC}\left(X \times_{Y} X\right)\right) \simeq \mathrm{QC}(\mathcal{L} Y)
$$

in which the central functor

$$
\mathrm{QC}(\mathcal{L} Y) \simeq \mathcal{Z}\left(\mathrm{QC}\left(X \times_{Y} X\right)\right) \rightarrow \mathrm{QC}\left(X \times_{Y} X\right)
$$

is given by pullback and pushforward along the correspondence

$$
\mathcal{L} Y \longleftarrow \mathcal{L} Y \times_{Y} X \longrightarrow X \times_{Y} X .
$$

When $p: X \rightarrow Y$ is also proper with invertible dualizing complex, we deduce an analogous statement identifying the trace of $\mathrm{QC}\left(X \times_{Y} X\right)$ with $\mathrm{QC}(\mathcal{L} Y)$, conditional on the validity of Grothendieck duality in the derived setting.

Remark 1.10. One can find a precursor to the above in the work of Müger $[\mathrm{M}]$ and Ostrik Os.

Given a semisimple abelian monoidal category $\mathcal{C}$ and a module category $M$, consider the monoidal category $\mathcal{C}_{M}^{*}$ consisting of $\mathcal{C}$-linear endofunctors of $M$. Then independently of $M$, there is a canonical identification of the Drinfeld centers of $\mathcal{C}_{M}^{*}$ and $\mathcal{C}$.

A motivating example is when $H \subset G$ are finite groups, and one takes $\mathcal{C}=$ $\operatorname{Rep}(G)$ and $M=\operatorname{Rep}(H)$, so that $\mathcal{C}_{M}^{*} \simeq \operatorname{Vect}(H \backslash G / H)$. Then independently of $H$, the center of $\operatorname{Vect}(H \backslash G / H)$ is the category of adjoint equivariant vector bundles on $G$.

The above theorem extends this picture from finite groups to algebraic groups.

1.4.1. Example: Affine Hecke categories. As an illustration, we briefly mention a concrete application of the above theorem to a fundamental object in geometric representation theory.

Fix a reductive group $G$, and consider the Grothendieck-Springer resolution $\tilde{G} \rightarrow$ $G$ of pairs of a group element and a Borel subgroup containing it. Let $\mathcal{H}_{G}^{\text {aff }}$ be the quasi-coherent affine Hecke $\infty$-category of $G$-equivarant quasi-coherent sheaves on the Steinberg variety

$$
\mathcal{S} t_{G}=\tilde{G} \times_{G} \tilde{G}
$$


Work of Bezrukavnikov $\mathrm{Be}$ and others places $\mathcal{H}_{G}^{\text {aff }}$ at the heart of many recent developments in geometric representation theory.

Let us apply the above theorem with $X=\tilde{G} / G$ and $Y=G / G=\mathcal{L} B G$, where $G$ acts via conjugation. Observe that the iterated loop space $\mathcal{L} Y=\mathcal{L}(G / G)=$ $\mathcal{L}(\mathcal{L} B G)$ is nothing more than the derived moduli stack

$$
\mathcal{L}_{0 c_{G}}\left(T^{2}\right)=\operatorname{Map}\left(T^{2}, B G\right)
$$

of $G$-local systems on the torus. Concretely, $\mathcal{L}_{o c_{G}}\left(T^{2}\right)$ is the "commuting variety" (or rather, commuting derived stack) parameterizing pairs of commuting elements in $G$ up to simultaneous conjugation.

Corollary 1.11. There is a canonical equivalence

$$
\mathcal{Z}\left(\mathcal{H}_{G}^{\text {aff }}\right) \simeq \mathrm{QC}(\mathcal{L}(\mathcal{L} B G)) \simeq \mathrm{QC}\left(\left(B G^{S^{1}}\right)^{S^{1}}\right) \simeq \mathrm{QC}\left(\mathcal{L}_{o c_{G}}\left(T^{2}\right)\right)
$$

between the center of the quasi-coherent affine Hecke $\infty$-category and the $\infty$-category of sheaves on the derived moduli stack of G-local systems on the torus.

A similar statement holds replacing the Grothendieck-Springer resolution $\tilde{G} \rightarrow G$ by the Springer resolution $T^{*} G / B \rightarrow \mathcal{N}$ of the nilpotent cone. In this case, the center is equivalent to the $\infty$-category of sheaves on the derived stack of pairs of a nilpotent and a commuting group element up to simultaneous conjugation. In this linear version, one can also work $\mathbb{G}_{m}$-equivariantly via the natural dilation action.

Remark 1.12. We will not return to specific applications to representation theory in this paper, but the interested reader will find further results along these lines in the paper BN2 which studies integral transforms in the context of $\mathcal{D}$-modules. It includes applications to the more familiar Hecke categories $\mathcal{D}(B \backslash G / B)$ of $\mathcal{D}$ modules on flag varieties. In particular, their Drinfeld centers are identified with character sheaves on $G$, resulting in a Langlands duality for character sheaves.

1.5. Topological field theory. As we discuss in Section 6, our results may be viewed from the perspective of topological field theory.

The $\infty$-category $\mathrm{QC}(\mathcal{L} X)$ (the Drinfeld center $\mathcal{Z}(\mathrm{QC}(X))$ ) carries a rich collection of operations generalizing the braided tensor structure on modules for the classical Drinfeld double. Namely, for any cobordism $\Sigma$ between disjoint unions of circles $\left(S^{1}\right)^{\amalg m}$ and $\left(S^{1}\right)^{\amalg n}$, we obtain restriction maps between the corresponding mapping stacks

$$
(\mathcal{L} X)^{\times m} \leftarrow X^{\Sigma} \rightarrow(\mathcal{L} X)^{\times n} .
$$

Pullback and pushforward along this correspondence defines a functor

$$
\mathrm{QC}(\mathcal{L} X)^{\otimes m} \rightarrow \mathrm{QC}(\mathcal{L} X)^{\otimes n}
$$

which is compatible with composition of cobordisms. In particular, we obtain a map from the configuration space of $m$ small disks in the standard disk to functors:

$$
\mathrm{QC}(\mathcal{L} X)^{\otimes m} \rightarrow \mathrm{QC}(\mathcal{L} X) .
$$

This equips $\mathrm{QC}(\mathcal{L} X)$, and hence the Drinfeld center $\mathcal{Z}(\mathrm{QC}(X))$, with the structure of a (framed) $\mathcal{E}_{2}$-category. This establishes the categorified (cyclic) Deligne conjecture in the current geometric setting.

In particular for $X=B G, X^{\Sigma}$ is the derived moduli stack of $G$-local systems on $\Sigma$ and we obtain a topological gauge theory. 
More generally, we have the following corollary of our main results:

Corollary 1.13. Let $X$ be a perfect stack, and let $\Sigma$ be a finite simplicial set. Then there is a canonical equivalence $\mathrm{QC}\left(X^{\Sigma}\right) \simeq \mathrm{QC}(X) \otimes \Sigma$, where $X^{\Sigma}=\operatorname{Map}(\Sigma, X)$ denotes the derived mapping stack, and $-\otimes \Sigma$ the tensor of stable $\infty$-categories over simplicial sets.

One can view this corollary as providing for a TFT over finite simplicial sets. We assign to such a simplicial set $U$ the $\infty$-category $\mathrm{QC}\left(X^{U}\right)$, and for any diagram of finite simplicial sets

$$
U \rightarrow \Sigma \leftarrow V
$$

(for example, a cobordism of manifolds), we obtain a correspondence of mapping stacks

$$
X^{U} \leftarrow X^{\Sigma} \rightarrow X^{V}
$$

and hence by pullback and pushforward a functor

$$
\mathrm{QC}\left(X^{U}\right) \rightarrow \mathrm{QC}\left(X^{V}\right),
$$

satisfying composition laws with respect to gluings.

In particular, since $\mathrm{QC}\left(X^{S^{n}}\right)$ is equivalent to the $\mathcal{E}_{n}$-center $\mathcal{Z}_{\mathcal{E}_{n}}(\mathrm{QC}(X))$, we obtain an action of the (framed) $\mathcal{E}_{n+1}$-operad on $\mathcal{Z}_{\mathcal{E}_{n}}(\mathrm{QC}(X))$. This establishes the categorified (cyclic) Kontsevich conjecture in the current geometric setting.

1.5.1. Extended TFT. An exciting recent development (postdating the submission of this paper) is Jacob Lurie's announced proof of the Cobordism Hypothesis [L6] which characterizes extended TFTs in arbitrary dimensions. The results of this paper may be used to prove that the monoidal $\infty$-category $\mathrm{QC}(X)$, for a perfect stack $X$, defines an extended two-dimensional TFT. We briefly summarize this here (see BN2 for more details regarding an analogous extended two-dimensional TFT).

The extended two-dimensional TFT $\mathcal{Z}_{X}$ associated to a perfect stack $X$ is a symmetric monoidal functor

$$
\mathcal{Z}_{X}: 2 \text { Bord } \rightarrow 2 \mathrm{Alg}
$$

from the $(\infty, 2)$-category of unoriented bordisms:

- objects: 0-manifolds,

- 1-morphisms: 1-dimensional bordisms between 0-manifolds,

- 2-morphisms: classiying spaces of 2-dimensional bordisms between 1-bordisms,

to the Morita $(\infty, 2)$-category of algebras $2 A l g$ :

- objects: algebra objects in stable presentable $\infty$-categories,

- 1-morphisms: bimodule objects in stable presentable $\infty$-categories,

- 2-morphisms: classifying spaces of morphisms of bimodules.

Note that given $A \in 2 A l g$, we can pass to the $(\infty, 2)$-category of $\operatorname{modules} \operatorname{Mod}_{A}$, and bimodules correspond to functors between $(\infty, 2)$-categories of modules. Thus if a field theory assigns $A$ to a point, we can also think of it as assigning $\operatorname{Mod}_{A}$ to a point.

The field theory $\mathcal{Z}_{X}$ assigns the following to closed 0,1 and 2-manifolds:

- To a point, $\mathcal{Z}_{X}$ assigns the monoidal $\infty$-category $\mathrm{QC}(X)$, or equivalently, the $(\infty, 2)$-category of $\mathrm{QC}(X)$-linear $\infty$-categories. 
- To a circle, $\mathcal{Z}_{X}$ assigns the Hochschild homology category of $\mathrm{QC}(X)$, which by our results can be identified with $\mathrm{QC}(\mathcal{L} X)$.

- To a closed surface $\Sigma, \mathcal{Z}_{X}$ assigns the $k$-module of derived global sections $\Gamma\left(X^{\Sigma}, \mathcal{O}_{X^{\Sigma}}\right)$ of the structure sheaf of the mapping space $X^{\Sigma}$.

The proof that $\mathrm{QC}(X)$ satisfies the dualizability conditions of [L6] to define an extended TFT follows closely from the identification of the Hochschild homology and cohomology categories of $\mathrm{QC}(X)$ (which is a necessary consequence of the TFT structure). One could consult [BN2] for more details in an analogous setting.

1.5.2. Rozansky-Witten theory. The topological field theory $\mathcal{Z}_{X}$ introduced above is closely related to well-known three-dimensional topological field theories. When the target $X$ is the classifying stack $B G$ of a finite group, $\mathcal{Z}_{X}$ is the untwisted version of Dijkgraaf-Witten theory. More generally, if $X$ is a 2-gerbe $\left(B B \mathbb{G}_{m^{-}}\right.$ torsor) over $B G$ (classified by a class in $H^{3}\left(G, \mathbb{G}_{m}\right)$ ), we obtain the twisted version (as studied in $[\mathrm{Fr}]$ ).

When $X$ is a smooth complex projective variety, $\mathcal{Z}_{X}$ is closely related to the $\mathbb{Z} / 2$-graded three-dimensional topological field theory associated to the holomorphic symplectic manifold $T^{*} X$ by Rozansky-Witten [RW], Kontsevich [K2 and Kapranov Kap. In particular, see RobW] and [KRS for work on RozanskyWitten theory as an extended topological field theory.

We confine ourselves here to a brief comparison of the two theories on the circle. On the one hand, $\mathcal{Z}_{X}$ assigns to $S^{1}$ the stable $\infty$-category $\mathrm{QC}(\mathcal{L} X)$. Since $X$ is a smooth scheme, $\mathcal{L} X$ is the total space $T_{X}[-1]=\operatorname{Spec} \operatorname{Sym}{ }^{\bullet} \Omega_{X}[1]$ of the shifted tangent bundle of $X$. Thus we can identify $\mathrm{QC}(\mathcal{L} X)$ with module objects in the $\infty$-category $\mathrm{QC}(X)$ for the commutative algebra object $\mathrm{Sym}^{\bullet} \Omega_{X}[1]$. Via Koszul duality, this category is closely related to modules for $\mathrm{Sym}^{\bullet} T_{X}[-2]$, or in other words, sheaves on $T^{*} X$ with an unusual grading. On the other hand, RozanskyWitten theory assigns to $S^{1}$ the $\infty$-category $\operatorname{Perf}\left(T^{*} X\right)$ of perfect complexes. It would be very interesting to develop Rozansky-Witten theory as a fully extended TFT using the results of [L6], and explore its relation with the derived algebraic geometry of $\mathrm{QC}(X)$.

\section{Preliminaries}

In this section, we summarize relevant technical foundations in the theory of $\infty$ categories and derived algebraic geometry. It is impossible to describe this entire edifice in a few pages, so we will aim to highlight the particular concepts, results and their references that will play a role in what follows.

2.1. $\infty$-categories. We are interested in studying algebraic operations on categories of a homotopy-theoretic nature such as derived categories of sheaves or spectra. It is well established that the theory of triangulated categories, through which derived categories are usually viewed, is inadequate to handle many basic algebraic and geometric operations. Examples include the absence of a good theory of gluing or of descent, of functor categories, or of generators and relations. The essential problem is that passing to homotopy categories discards essential information (in particular, homotopy coherent structures, homotopy limits and homotopy colimits).

This information can be captured in many alternative ways, the most common of which is the theory of model categories. Model structures keep weakly equivalent objects distinct but retain the extra structure of resolutions which enables the 
formulation of homotopy coherence. This extra structure can be very useful for calculations but makes some functorial operations difficult. In particular, it can be hard to construct certain derived functors because the given resolutions are inadequate. There are also fundamental difficulties with the consideration of functor categories between model categories. However, much of the essential information encoded in model categories can be captured by the Dwyer-Kan simplicial localization. This construction uses the weak equivalences to construct simplicial sets (or alternatively, topological spaces) of maps between objects, refining the sets of morphisms in the underlying homotopy category (which are recovered by passing to $\pi_{0}$ ).

This intermediate regime between model categories and homotopy categories is encoded by the theory of $(\infty, 1)$-categories, or simply $\infty$-categories. The notion of $\infty$-category captures (roughly speaking) the notion of a category whose morphisms form topological spaces and whose compositions and associativity properties are defined up to coherent homotopies. Thus an important distinction between $\infty$ categories and model categories or homotopy categories is that coherent homotopies are naturally built in to all the definitions. Thus for example all functors are naturally derived and the natural notions of limits and colimits in the $\infty$-categorical context correspond to homotopy limits and colimits in more traditional formulations.

The theory of $\infty$-categories has many alternative formulations (as topological categories, Segal categories, quasi-categories, etc; see Ber for a comparison between the different versions). We will follow the conventions of [L2, which is based on Joyal's quasi-categories [Jo. Namely, an $\infty$-category is a simplicial set, satisfying a weak version of the Kan condition guaranteeing the fillability of certain horns. The underlying simplicial set plays the role of the set of objects while the fillable horns correspond to sequences of composable morphisms. The book L2] presents a detailed study of $\infty$-categories, developing analogues of many of the common notions of category theory (an overview of the $\infty$-categorical language, including limits and colimits, appears in [L2, Chapter 1.2]).

Among the structures we will depend on are the $\infty$-category of $\infty$-categories [L2, 3], adjoint functors [L2, 5.2], and ind-categories and compact objects L2, 5.3] (see also Section 3.1). Most of the objects we encounter form presentable $\infty$ categories [L2, 5.5]. Presentable $\infty$-categories are $\infty$-categories which are closed under all (small) colimits (as well as limits, L2, Proposition 5.5.2.4]) and moreover are generated in a weak sense by a small category. In particular, by a result of Simpson [L2, Theorem 5.5.1.1], they are given by suitable localizations of $\infty$ categories of presheaves on a small $\infty$-category. Presentable $\infty$-categories form an $\infty$-category $\mathcal{P} r^{\mathrm{L}}$ whose morphisms are continuous functors, that is, functors that preserve all colimits [L2, 5.5.3]. Note that since presentable categories are closed under all (co)products, categories with a finiteness condition (such as compact spaces, coherent sheaves, etc.) do not fall under this rubric. A typical example is the $\infty$-category of spaces.

Algebra (and algebraic geometry, see below) in the $\infty$-categorical (or derived) setting has been developed in recent years by Toën-Vezzosi ToVe1, ToVe2 and Lurie L3, L4, L5. This has resulted in a very powerful and readily applicable formalism, complete with $\infty$-analogues of many of the common tools of ordinary category theory. We single out two powerful tools that are crucial for this paper 
and available in the $\infty$-context thanks to [L4] (but are not available in a suitable form in the triangulated or model contexts):

- The general theory of descent, as embodied by the $\infty$-categorical version of the Barr-Beck theorem ([L4, Theorem 3.4.5]).

- The theory of tensor products of and functor categories between presentable $\infty$-categories (reviewed in Section 2.2).

2.1.1. Enhancing triangulated categories. The $\infty$-categorical analogue of the additive setting of homological algebra is the setting of stable $\infty$-categories [L3]. A stable $\infty$-category can be defined as an $\infty$-category with a zero-object, closed under finite limits and colimits, and in which pushouts and pullbacks coincide [L3, 2,4]. The result of [L3, 3] is that stable categories are enhanced versions of triangulated categories, in the sense that the homotopy category of a stable $\infty$-category has the canonical structure of a triangulated category. We will mostly be concerned with $\infty$-categories that are both presentable and stable, as studied in [L3, 17]. Typical examples are the $\infty$-categorical enhancements of the derived categories of modules over a ring, quasi-coherent sheaves on a scheme, and the $\infty$-category of spectra.

Given a triangulated category which is linear over a ring $k$, we may consider enhancing its structure in three different ways, promoting it to

- a differential graded (dg) category,

- an $A_{\infty}$-category, or

- a stable $\infty$-category.

Among the many excellent references for $\mathrm{dg}$ and $A_{\infty}$-categories, we recommend the survey [Ke. Relative to a ring of characteristic zero $k$, all three formalisms become equivalent: $k$-linear stable $\infty$-categories are equivalent to $k$-linear pre-triangulated $\mathrm{dg}$ categories (that is, those whose homotopy category is triangulated). Thus we recommend that the reader interested in characteristic zero applications substitute the term "pretriangulated $k$-linear dg category" for "stable $\infty$-category" throughout the present paper. The distinction between $k$-linear stable $\infty$-categories, dgand $A_{\infty}$-categories becomes important when considering commutative algebra away from characteristic zero, for which purpose we will only consider the former.

2.2. Monoidal $\infty$-categories. The definitions and results of this paper depend in an essential way on noncommutative and commutative algebra for $\infty$-categories, as developed by Lurie in [L4] and [L5]. We briefly summarize this theory in this section, giving detailed references for the benefit of the reader.

The definition of a monoidal $\infty$-category is given in [L4, 1.1]. The homotopy category of a monoidal $\infty$-category is an ordinary monoidal category. The $\infty$ categorical notion incorporates not only the naive notion of multiplication and unit on an $\infty$-category $\mathcal{C}$ but also all of the higher coherences for associativity, which are packaged in the data of a fibration over $\Delta^{\mathrm{op}}$ whose fiber over $[n]$ is $\mathcal{C}^{\times n}$. (Alternatively, it can be captured concretely by a bisimplicial set with compatibilities, or as a monoid object in $\mathcal{C}$ [L4, Remark 1.2.15], that is, a simplicial object in $\mathcal{C}$ mimicking the classifying space of a monoid.) An algebra object in $\mathcal{C}$ can then be defined [L4, 1.1.14] as an appropriate section of this fibration. The $\infty$-categorical notion of algebra reduces to the more familiar notions of $A_{\infty} k$-algebra or $A_{\infty}$-ring spectrum when the ambient monoidal $\infty$-category is that of differential graded $k$-modules or that of spectra [L4, 4.3]. In other words, it encodes a multiplication associative up to coherent homotopies. Likewise, (left) modules over an algebra are defined by the 
same simplicial diagrams as algebras, except with an additional marked vertex at which we place the module [L4, 2.1]. There is also a pairing to $\mathcal{C}$ between left and right modules over an algebra object $A$ in $\mathcal{C}$, namely, the relative tensor product - $\otimes_{A} \cdot$ defined by the two-sided bar construction [L4, 4.5]. Monoidal $\infty$-categories, algebra objects in a monoidal category, and module objects over an algebra object themselves form $\infty$-categories, some of whose properties (in particular behavior of limits and colimits) are worked out in [L4, 1,2]. In particular, limits of algebra objects are calculated on the underlying objects [L4, 1.5] and module categories are stable [L4, Proposition 4.4.3].

The definition of a symmetric monoidal $\infty$-category is given in [L5, 1], modeled on the Segal machine for infinite loop spaces. Namely, we replace $\Delta^{\text {op }}$ in the definition of monoidal $\infty$-categories by the category of pointed finite sets, thus encoding all the higher compatibilities of commutativity. Likewise, commutative algebra objects are defined as suitable sections of the defining fibration. On the level of homotopy categories, we recover the notion of commutative algebra object in a symmetric monoidal category, but on the level of chain complexes or spectra, this notion generalizes the notion of $\mathcal{E}_{\infty}$-algebra or $\mathcal{E}_{\infty}$-ring spectrum (as developed in EKMM , HSS ). An important feature of the $\infty$-category of commutative algebra objects is that coproducts of commutative algebra objects are calculated by the underlying monoidal structure [L5, Proposition 4.7]. Section [L5, 5] introduces commutative modules over commutative algebra objects $A$, which are identified with both left and right modules over the underlying algebras. The key feature of the $\infty$-category of $A$-modules is that it has a canonical symmetric monoidal structure [L5, Proposition 5.7], extending the relative tensor product of modules. Moreover, commutative algebras for this structure are simply commutative algebras over $A$ [L5, Proposition 5.9].

One of the key developments of [L4] is the $\infty$-categorical version of tensor products of abelian categories [De. Namely, in [L4, 4.1], it is shown that the $\infty$-category $\mathcal{P r}{ }^{\mathrm{L}}$ of presentable $\infty$-categories has a natural monoidal structure. In this structure, the tensor product $\mathcal{C} \otimes \mathcal{D}$ of presentable $\mathcal{C}, \mathcal{D}$ is a recipient of a universal functor from the Cartesian product $\mathcal{C} \times \mathcal{D}$ which is "bilinear" (commutes with colimits in each variable separately). Moreover, [L5, Proposition 6.18] lifts this to a symmetric monoidal structure in which the unit object is the $\infty$-category of spaces. This structure is in fact closed, in the sense that $\mathcal{P} r^{\mathrm{L}}$ has an internal hom functor compatible with the tensor structure; see [L2, Remark 5.5.3.9] and [L4, Remark 4.1.6]. The internal hom assigns to presentable $\infty$-categories $\mathcal{C}$ and $\mathcal{D}$ the $\infty$-category of colimit-preserving functors $\operatorname{Fun}^{\mathrm{L}}(\mathcal{C}, \mathcal{D})$, which is presentable by L2, Proposition 5.5.3.8]. In Section 5.1 below, we use the monoidal structure on $\mathcal{P} r^{\mathrm{L}}$ to define an analogue for $\infty$-categories of the Hochschild cohomology of algebras or topological Hochschild cohomology of ring spectra.

The symmetric monoidal structure on the $\infty$-category $\mathcal{P} r^{\mathrm{L}}$ of presentable $\infty$ categories restricts to one on the full $\infty$-subcategory of stable presentable $\infty$ categories ([L4, 4.2] and [L5, 6.22]). The unit of the restricted monoidal structure is the stable category of spectra. In particular, this induces a symmetric monoidal structure on spectra and exhibits presentable stable categories as tensored over spectra. Thus if $\mathcal{C}$ is a symmetric monoidal stable $\infty$-category (that is, a stable commutative ring object in stable $\infty$-categories), we may consider module categories over $\mathcal{C}$. These modules themselves will form a symmetric monoidal $\infty$-category 
under the operation $\otimes_{\mathcal{C}}$, which is characterized by the two-sided bar construction with respect to $\mathcal{C}$. In our applications, $\mathcal{C}$ will be the $\infty$-category $\mathrm{QC}(Y)$ of quasicoherent sheaves on a derived stack $Y$, and we will consider the tensor products of module categories of the form $\mathrm{QC}(X)$ for derived stacks $X \rightarrow Y$.

In Section [5.1] we use this general formalism to define the center (or Hochschild cohomology) and universal trace (or Hochschild homology) for algebra objects in any symmetric monoidal $\infty$-category. The case of spectra recovers topological Hochschild (co)homology, while the case of presentable $\infty$-categories $\mathcal{P}{ }^{\mathrm{L}}$ provides a derived generalization of the Drinfeld center and will be the focus of our applications in Section 5 .

In Section 4.1 we discuss basic properties of $\infty$-categories of modules and the tensor product of small stable $\infty$-categories.

2.3. Derived algebraic geometry. Algebraic geometry provides a wealth of examples of symmetric monoidal categories, as well as powerful tools to study such categories. To a scheme or stack, we may assign its category of quasi-coherent sheaves with its commutative multiplication given by the tensor product. This construction generalizes the category of $R$-modules for $R$ a commutative ring (the case of $\operatorname{Spec} R$ ), and the category of representations of an algebraic group $G$ (the case of classifying stacks $B G$ ). Conversely, Tannakian formalism often allows us to reverse this process and assign a stack to a symmetric monoidal category.

Schemes and stacks are also natural sources of symmetric monoidal $\infty$-categories, which refine the familiar symmetric monoidal derived categories of quasi-coherent sheaves. For example, to any stack in characteristic zero, we can assign the differential graded enhancement of its derived category, constructed by taking the differential graded category of complexes with quasi-coherent cohomology and localizing the quasi-isomorphisms (following $\mathrm{Ke}, \mathrm{D}, \mathrm{To1}$ ). In general, we can consider the stable symmetric monoidal $\infty$-category of quasi-coherent sheaves on any stack whose homotopy category is the familiar derived category.

Trying to geometrically describe algebraic operations on $\infty$-categories of quasicoherent sheaves quickly takes us from ordinary algebraic geometry to an enhanced version, which has been developed over the last few years, known as derived algebraic geometry [L1, L3, L4, L5, ToVe1, ToVe2. In fact, derived algebraic geometry also provides a far greater abundance of examples of stable symmetric monoidal $\infty$-categories. Thus it is most natural to both ask and answer algebraic questions about stable symmetric monoidal $\infty$-categories in this context.

Derived algebraic geometry generalizes the world of schemes simultaneously in two directions. Regarding schemes in terms of their functors of points, which are functors from rings to sets (satisfying a sheaf axiom with respect to a Grothendieck topology), we want to replace both the source and the target categories by suitable $\infty$-categories. First, we may consider functors from rings to spaces (or equivalently, simplicial sets) considered as an $\infty$-category (with weak homotopy equivalences inverted). If we consider only 1-truncated spaces, or equivalently their fundamental groupoids, we recover the theory of stacks. This naturally leads to the introduction of higher stacks where we consider sheaves of (not necessarily 1-truncated) spaces on the category of rings. For example, we can take any space and consider it as a higher stack by taking (the sheafification of) the corresponding constant functor on rings. 
To pass from higher stacks to derived stacks, we replace the source category of commutative rings by an $\infty$-category of commutative ring objects in a symmetric monoidal $\infty$-category. There are at least three natural candidates that are commonly considered:

(1) connective (that is, homological or nonpositively graded) commutative differential graded $k$-algebras over a commutative ring $k$ of characteristic zero;

(2) simplicial commutative rings, or simplicial commutative $k$-algebras over a commutative ring $k$;

(3) connective $\mathcal{E}_{\infty}$-ring spectra, or connective $\mathcal{E}_{\infty}$-algebras over the EilenbergMacLane spectrum $H(k)$ of a commutative $\operatorname{ring} k 1$

When $k$ is a $\mathbb{Q}$-algebra, the $\infty$-categories of connective differential graded $k$ algebras, simplicial commutative $k$-algebras, and connective $\mathcal{E}_{\infty}$-algebras over $H(k)$ are all equivalent. For a general commutative ring $k$, simplicial commutative rings provide a setting for importing notions of homotopy theory into algebraic geometry over $k$ (for example, for the aim of describing centers of $\infty$-categories of sheaves on schemes or stacks). Connective $\mathcal{E}_{\infty}$-ring spectra are more subtle and provide the setting for importing algebro-geometric notions back into stable homotopy theory. General $\mathcal{E}_{\infty}$-ring spectra provide a radical generalization which is at the heart of stable homotopy theory. (See $[\mathrm{Sh}]$ and the references therein for the relation of algebra over rings and over the corresponding Eilenberg-MacLane spectra.)

The techniques and results of this paper apply equally in any of the three settings, and we will refer to any of the three as commutative derived rings without further comment.

Roughly speaking, a derived stack is a functor from the $\infty$-category of commutative derived rings to the $\infty$-category of topological spaces. It should satisfy a sheaf property with respect to a Grothendieck topology on commutative derived rings. Examples of derived stacks include:

(1) spaces (constant functors),

(2) ordinary schemes and stacks,

(3) the spectrum of a derived commutative ring (the corresponding representable functor),

(4) objects obtained by various gluings or quotients (colimits) and intersections or fiber products (limits) of the above examples.

We now recall the precise definitions of derived stacks, following [ToVe1, ToVe2, To2.

The opposite of the $\infty$-category $\mathcal{A} l g_{k}$ of derived commutative $k$-algebras admits a Grothendieck topology with respect to étale morphisms. For $A, B \in \mathcal{A} l g_{k}$, a morphism $A \rightarrow B$ is étale if the induced morphism on the connected component $\pi_{0}(A) \rightarrow \pi_{0}(B)$ is étale, and for $i>0$, the induced map on higher homotopy groups is an isomorphism

$$
\pi_{i}(A) \otimes_{\pi_{0}(A)} \pi_{0}(B) \stackrel{\sim}{\longrightarrow} \pi_{i}(B) .
$$

A finite family of morphisms $\left\{f_{i}: A \rightarrow B_{i}\right\}$ is an étale covering if each $f_{i}$ is étale and the induced morphism is surjective:

$$
\amalg \operatorname{Spec} \pi_{0}\left(B_{i}\right) \longrightarrow \operatorname{Spec} \pi_{0}(A) .
$$

\footnotetext{
${ }^{1}$ Note that by the results of [L5] reviewed above, an $\mathcal{E}_{\infty}$-algebra over $H(k)$ is equivalently a commutative algebra object in the symmetric monoidal $\infty$-category of $H(k)$-module spectra.
} 
This induces a Grothendieck topology on the (opposite of the) homotopy category of $\mathcal{A} l g_{k}$.

Now a derived stack $X$ is a covariant functor from $\mathcal{A} l g_{k}$ to the $\infty$-category $\mathcal{T}$ op of topological spaces which is a sheaf with respect to the étale topology. In particular, for any étale cover $A \rightarrow B$, the induced morphism

$$
X(A) \stackrel{\sim}{\longrightarrow} \lim X\left(B^{*}\right)
$$

is an equivalence, where $B^{*}$ is the standard cosimplicial resolution of $A$ with $i$ th simplex $B^{\otimes_{A} i+1}$.

We will work exclusively with derived stacks whose diagonal morphism $\Delta: X \rightarrow$ $X \times X$ is representable and affine. Given a quasi-compact derived stack $X$ with affine diagonal, we can choose a cover $U \rightarrow X$ by an affine derived scheme, and obtain a C̈ech simplicial affine derived scheme $U_{*} \rightarrow X$ with $k$-simplices given by the $k$-fold fiber product $U \times_{X} \cdots \times_{X} U$ and whose geometric realization is equivalent to $X$.

2.4. Derived loop spaces. Even if we are interested in studying primarily schemes, the world of derived stacks is a necessary setting in which to calculate homotopically correct quotients, fiber products and mapping spaces. We illustrate this with an important geometric operation on derived stacks: the formation of the derived loop space. This operation is one of our motivations for considering derived stacks in the first place, since the derived loop space of an ordinary scheme or stack is already a nontrivial derived stack. (See [BN1] for a different appearance of derived loop spaces in relation to cyclic homology, $\mathcal{D}$-modules and representation theory.)

The free loop space of a derived stack $X$ is the internal hom $\mathcal{L} X=X^{S^{1}}=$ $\operatorname{Map}\left(S^{1}, X\right)$ of maps from the constant stack given by the circle $S^{1}$. As a derived stack, the loop space may be described explicitly as the collection of pairs of points in $X$ with two paths between them, or in other words, as the derived self-intersection of the diagonal

$$
\mathcal{L} X \simeq X \times_{X \times X} X
$$

Let us illustrate this notion in a few examples.

For $X$ a topological space (constant stack), $\mathcal{L} X$ is of course the free loop space of $X$ (again considered as a constant stack).

For $X=B G$ the classifying space of an algebraic group, $\mathcal{L} X=G / G$ is the adjoint quotient of $G$, or in other words, the adjoint group for the universal bundle $E G=\mathrm{pt} \rightarrow B G$. Note that this agrees with the underived inertia stack of $B G$.

For $X$ a smooth variety over a field of characteristic zero, the derived selfintersection of the diagonal can be calculated by a Koszul complex to be the spectrum of the complex of differential forms on $X$ (with zero differential) placed in homological degrees. We thus obtain that the loop space of a smooth variety $X$ can be identified with the relative spectrum of the symmetric algebra of the shifted tangent bundle $T_{X}[-1]$.

Similarly, for any $n \geq 0$, we may consider the derived stack of maps $X^{S^{n}}=$ $\operatorname{Map}\left(S^{n}, X\right)$ from the $n$-sphere $S^{n}$ into $X$. Concretely, the presentation of $S^{n}$ by 
two cells glued along $S^{n-1}$ leads to an iterated description of $X^{S^{n}}$ as the selfintersection

$$
X^{S^{n}}=X \times_{X^{S^{n-1}}} X
$$

along two copies of constant maps over $X^{S^{n-1}}$.

More generally, for any topological space $\Sigma$ and stack $X$ we can consider the derived mapping stack $X^{\Sigma}=\operatorname{Map}(\Sigma, X)$. As we will demonstrate, this construction suggests that the world of derived algebraic geometry is a natural setting in which to construct topological $\sigma$-models.

2.5. $\mathcal{E}_{n}$-structures. When considering monoidal structures on vector spaces, we have the option of considering associative or commutative multiplications. When considering monoidal structures on categories, we are faced with three levels of increasing commutativity: "plain" monoidal categories carrying an associative multiplication; braided monoidal categories, in which there is a functorial isomorphism exchanging the order of the multiplication $A \otimes B \rightarrow B \otimes A$ and satisfying the braid relations; symmetric monoidal categories, in which the square of the braiding is the identity.

In homotopy theory, there is an infinite sequence of types of algebraic structures interpolating between associativity and commutativity, modeled on the increasing commutativity of $n$-fold iterated loop spaces. These algebraic structures can be encoded by the little $n$-disk or $\mathcal{E}_{n}$-operad for $n \geq 1$ or $n=\infty$, which is an operad in the category of topological spaces. Recall that an operad $F$ (in spaces) is a sequence of spaces $F(k)$, which parametrize $k$-fold multiplication operations in the algebraic structures we are encoding, together with actions of the symmetric group permuting the entries and equivariant composition maps. The $k$-th space of the $\mathcal{E}_{n}$-operad parametrizes disjoint collections of $k$ small balls in the $n$-ball, with the natural "picture-in-picture" composition maps. An $\mathcal{E}_{n}$-vector space carries operations labelled by components of the $\mathcal{E}_{n}$-operad and is an associative algebra for $n=1$ and a commutative algebra for $n>1$. The notion of $\mathcal{E}_{n}$-category for a (usual discrete) category is sensitive to the fundamental groupoid of the $\mathcal{E}_{n}$-operad, leading to the three different notions of monoidal category $(n=1)$, braided monoidal category $(n=2)$ and symmetric monoidal category $(n>2$, since for $n>2$ the spaces in the $\mathcal{E}_{n}$-operad are simply connected). However, even on the level of graded vector spaces, with operations labelled by the homology of the $\mathcal{E}_{n}$-operad, we obtain different notions for every $n$, with $n=2$ giving the notion of a Gerstenhaber algebra familiar from the study of Hochschild cohomology.

We have already encountered the notions of algebra object (corresponding to the case $\mathcal{E}_{1}$, or equivalently $A_{\infty}$ ) and commutative algebra object (corresponding to $\mathcal{E}_{\infty}$ ) in the context of a symmetric monoidal $\infty$-category, such as dg modules over a ring $R$, spectra or presentable $\infty$-categories. In [F1], the general theory of algebras over operads in $\infty$-categories is developed and applied to algebra and geometry in the $\mathcal{E}_{n}$-case. Roughly speaking, in the $\infty$-categorical context, we consider the operadic operations and compositions in a homotopy coherent fashion (see Section 5.3 for more details). Thus, for example, an $\mathcal{E}_{2}$-category is a homotopy-theoretic analogue of a braided monoidal category.

The notions of $\mathcal{E}_{n}$-algebras and categories pervade homotopy theory, but have also become prominent in algebra and topological field theory. We briefly mention two such applications. 
Deligne's Hochschild cohomology conjecture asserts that the Hochschild cochain complex of an associative algebra is an $\mathcal{E}_{2}$-algebra, lifting the Gerstenhaber algebra structure on Hochschild cohomology. Kontsevich's conjecture generalizes this to assert that the Hochschild cohomology of an $\mathcal{E}_{n}$-algebra is an $\mathcal{E}_{n+1}$-algebra.

The space of states associated to an $(n-1)$-sphere by an $n$-dimensional topological field theory has a natural $\mathcal{E}_{n}$-structure, given by tree-level field theory operations, independent of where the field theory takes its values (vector spaces, chain complexes, categories, etc.)

Remark 2.1. After the completion of this paper, the paper [L5] was revised to include a thorough treatment of $\infty$-categorical operads and their algebras. Furthermore, the paper [L7] studies in great detail the specific case of the $\mathcal{E}_{n}$-operads (including a proof of general versions of the Deligne-Kontsevich conjecture). We refer the reader to these preprints for details on these topics.

\section{Perfect stacks}

By an $\infty$-category, we will always mean an $(\infty, 1)$-category without further comment, and we refer to Section 2 for an overview of the required aspects of the general theory. For the reader accustomed to working with model categories, it is important to note that in an $\infty$-category, all tensors, homs, limits, colimits, and other usual operations are taken in a derived or homotopical sense. In other words, coherent homotopies are automatically built in to all definitions, though it may not explicitly appear in the notation. For example, a colimit in the $\infty$-category obtained as the localization of a model category corresponds to a homotopy colimit in the original model category.

Throughout the rest of the paper, we fix a derived commutative ring $k$ (in any of the three senses discussed in Section 2.3) and work relative to $k$. We use the phrase derived commutative $k$-algebra to refer to a commutative algebra object in $k$-modules.

For $k$ a commutative $\mathbb{Q}$-algebra, one can replace $k$-modules with chain complexes over $k$, derived commutative $k$-algebras with commutative differential graded $k$ algebras, and stable $k$-linear $\infty$-categories with pretriangulated $k$-linear differential graded categories.

In Section 3.1, we review various notions of when an $\infty$-category is finitely generated and introduce the class of perfect stacks to which our results apply. In Section 3.2. we establish base change and the projection formula for perfect morphisms. In Section 3.3. we show that many common classes of stacks are perfect.

3.1. Definition of perfect stacks. Our main objects of study are $\infty$-categories of quasi-coherent sheaves on derived stacks. For the foundations of derived stacks and quasi-coherent sheaves on them, we refer the reader to ToVe1, ToVe2, To2, (Note that a theory of descent for sheaves on higher stacks was developed previously by Hirschowitz and Simpson [HS.)

Given a derived stack $X$, we have the stable symmetric monoidal $\infty$-category $\mathrm{QC}(X)$ of quasi-coherent sheaves on $X$. To recall its construction, consider first a derived commutative $k$-algebra $A$ and the representable affine derived scheme $X=\operatorname{Spec} A$. In this case, one defines $\mathrm{QC}(X)$ to be the $\infty$-category of $A$-modules $\operatorname{Mod}_{A}$ (i.e., module objects over $A$ in $k$-modules). Its homotopy category is the unbounded derived category of $A$. 
In general, any derived stack $X$ can be written as a colimit of a diagram of affine derived schemes $X \simeq \operatorname{colim}_{U \in A f f}{ }_{X} U$. Then one defines $\mathrm{QC}(X)$ to be the limit (in the $\infty$-category of $\infty$-categories) of the corresponding diagram of $\infty$-categories

$$
\mathrm{QC}(X):=\lim _{U \in A f f / X} \mathrm{QC}(U) .
$$

One can think of an object $F \in \mathrm{QC}(X)$ as collections of quasi-coherent sheaves $\left.F\right|_{U}$ on the terms $U$ together with compatible identifications between their pullbacks under the diagram maps.

When $X$ is quasi-compact and has affine diagonal, by choosing an affine cover $U \rightarrow X$ with induced C̆ech simplicial affine derived scheme $U_{*} \rightarrow X$, we can realize $\mathrm{QC}(X)$ by a smaller limit, the totalization of the cosimplicial diagram $\mathrm{QC}\left(U_{*}\right)$.

An important feature of the $\infty$-category $\mathrm{QC}(X)$ is that it is cocomplete, that is, closed under all small colimits (or equivalently, since $\mathrm{QC}(X)$ is stable, all small coproducts). Nevertheless, it can be difficult to control $\mathrm{QC}(X)$ algebraically via reasonable generators. In general, it is convenient (and sometimes indispensable) to work with $\infty$-categories that are "generated by finite objects" in a suitable sense. Let us summarize well-known approaches to this idea. In a moment, we will provide a more detailed discussion.

There are two common notions of when a small $\infty$-subcategory $\mathcal{C}^{\circ}$ generates an $\infty$-category $\mathcal{C}$. On the one hand, we could ask that $\mathcal{C}$ be the inductive limit Ind $\mathcal{C}^{\circ}$. On the other hand, we could ask that in $\mathcal{C}$ the right orthogonal of $\mathcal{C}^{\circ}$ vanishes.

There are three common notions of when an object should be considered finite: perfect objects, dualizable objects, and compact objects, which refer respectively to the geometry, monoidal structure, and categorical structure of $\mathrm{QC}(X)$.

We now introduce the class of perfect stacks. We will check below that for perfect stacks, the above notions of generators and finite objects all coincide.

Definition 3.1. Let $A$ be a derived commutative ring. An $A$-module $M$ is perfect if lies in the smallest $\infty$-subcategory of $\operatorname{Mod}_{A}$ containing $A$ and closed under finite colimits and retracts. For a derived stack $X, \infty$-category $\operatorname{Perf}(X)$ is the full $\infty$ subcategory of $\mathrm{QC}(X)$ consisting of those sheaves $M$ whose restriction $f^{*} M$ to any affine $f: U \rightarrow X$ over $X$ is a perfect module.

Definition 3.2. A derived stack $X$ is said to be perfect if it has affine diagonal and the $\infty$-category $\mathrm{QC}(X)$ is the inductive limit

$$
\mathrm{QC}(X) \simeq \operatorname{Ind} \operatorname{Perf}(X)
$$

of the full $\infty$-subcategory $\operatorname{Perf}(X)$ of perfect complexes.

A morphism $X \rightarrow Y$ is said to be perfect if its fibers $X \times_{Y} U$ over affines $U \rightarrow Y$ are perfect.

See [L2, 5.3.5] for the construction of Ind-categories of $\infty$-categories and L3, 8 ], where it is shown that Ind-categories of stable $\infty$-categories are stable. Let us mention that in the Ind-category $\operatorname{Ind} \mathcal{C}$ of an $\infty$-category $\mathcal{C}$, morphisms between Ind-objects can be calculated via the expected formula

$$
\operatorname{Hom}_{\text {Ind } \mathcal{C}}\left(\lim _{\longrightarrow}\left[X_{i}\right],\left(\lim _{\longrightarrow}\left[Y_{j}\right]\right) \simeq \lim \operatorname{colim} \operatorname{Hom}_{\mathcal{C}}\left(X_{i}, Y_{j}\right) .\right.
$$

For the reader unaccustomed to Ind-categories, we will momentarily give an alternative formulation of a perfect stack in the more familiar language of compactly generated categories. 
We next proceed with a review of the various notions of generators and finite objects. In Section 3.2 we show that perfect morphisms satisfy base change and the projection formula. In Section 3.3. we show that the class of perfect stacks includes many common examples of interest and check that any morphism between perfect stacks is itself perfect.

3.1.1. Finite objects. We review here the three common notions of finite objects and their interrelations (see [L3, 17] and [L4, 4.7] for more details, as well as [BV, HPS, Ke, LMS among many other sources). We remind the reader that we are working in the context of $\infty$-categories, so constructions such as colimits correspond to homotopy colimits in the context of model categories.

\section{Definition 3.3.}

(1) An object $M$ of a stable $\infty$-category $\mathcal{C}$ is said to be compact if $\operatorname{Hom}_{\mathcal{C}}(M,-)$ commutes with all coproducts (equivalently, with all colimits).

(2) An object $M$ of a stable symmetric monoidal $\infty$-category $\mathcal{C}$ is said to be (strongly) dualizable if there is an object $M^{\vee}$ and unit and trace maps

$$
1 \stackrel{u}{\longrightarrow} M \otimes M^{\vee} \stackrel{\tau}{\longrightarrow} 1
$$

such that the composite map

$$
M \stackrel{u \otimes \mathrm{id}}{\longrightarrow} M \otimes M^{\vee} \otimes M \stackrel{\operatorname{id} \otimes \tau}{\longrightarrow} M
$$

is the identity.

Suppose $\mathcal{C}$ is a stable presentable $\infty$-category (such as $\mathrm{QC}(X)$ ). Then an object $M \in \mathcal{C}$ is compact if and only if maps from $M$ to any small coproduct factor through a finite coproduct. Furthermore, a functor $F: \mathcal{C} \rightarrow \mathcal{D}$ between stable presentable $\infty$-categories that preserves finite colimits preserves small colimits if and only if it preserves small coproducts. (See [L3, Proposition 17.1].)

In a closed symmetric monoidal $\infty$-category $\mathcal{C}($ such as $\mathrm{QC}(X))$, an object $M \in \mathcal{C}$ is dualizable if and only if there exists a coevaluation map

$$
1 \rightarrow M \otimes \mathcal{H o m}(M, 1)
$$

satisfying the appropriate conditions (since one already has an evaluation map). If an object $M \in \mathcal{C}$ is dualizable, then we can turn internal Hom from $M$ into the tensor product with $M^{\vee}$ in the sense that there is a canonical equivalence

$$
\mathcal{H o m}(M,-) \simeq M^{\vee} \otimes(-) .
$$

In particular, this implies that $\mathcal{H o m}(M,-)$ preserves colimits and $M \otimes-$ preserves limits:

$$
\begin{aligned}
M \otimes \lim N_{\alpha} & \simeq \operatorname{Hom}_{\mathcal{C}}\left(1_{\mathcal{C}}, M \otimes \lim N_{\alpha}\right) \simeq \operatorname{Hom}_{\mathcal{C}}\left(M^{\vee}, \lim N_{\alpha}\right) \\
& \simeq \lim \operatorname{Hom}_{\mathcal{C}}\left(M^{\vee}, N_{\alpha}\right) \simeq \lim M \otimes N_{\alpha} .
\end{aligned}
$$

It is enlightening to note the following characterization of dualizable objects, which parallels the definition of compact objects (but will not be used in this paper).

Lemma 3.4. Let $\mathcal{C}$ be a symmetric monoidal presentable stable $\infty$-category, whose monoidal structure distributes over colimits. An object $M$ of $\mathcal{C}$ is then dualizable if and only if tensoring with $M$ preserves all limits. 
Proof. The necessity of $M \otimes-$ preserving limits is noted above $(\mathcal{C}$ is closed by virtue of being presentable with monoidal structure distributing over colimits; see [L4, Proposition 2.1.12]). To demonstrate sufficiency, assume that $M \otimes-$ preserves limits and then consider the endofunctor of $\mathcal{C}$ defined by tensoring with $M$. By assumption on $M$ and $\mathcal{C}$, this functor preserves all limits and colimits. We may now apply the adjoint functor theorem of [L2 to deduce the existence of a left adjoint $F$ to $M \otimes-$. Denote by $M^{\vee}$ the value $F\left(1_{\mathcal{C}}\right)$ of $F$ applied to the unit of $\mathcal{C}$. The existence of unit and trace maps $1_{\mathcal{C}} \rightarrow M \otimes M^{\vee} \rightarrow 1_{\mathcal{C}}$ is now a particular instance of the unit and counit maps for this adjunction, which implies that $M$ and $M^{\vee}$ are in duality. Hence $M$ is dualizable.

In a general stable presentable symmetric monoidal $\infty$-category $\mathcal{C}$, the classes of compact and dualizable objects do not coincide. In particular, the monoidal unit $1 \in \mathcal{C}$ is always dualizable but not necessarily compact.

In the case of a derived stack $X$, the unit $\mathcal{O}_{X} \in \mathrm{QC}(X)$ is compact if and only if the global sections functor $\Gamma(X,-)$ preserves colimits. (This fails if the global sections $\Gamma\left(X, \mathcal{O}_{X}\right)$ are too big such as in the following examples: ind-schemes such as the formal disk Spf $k[[t]]$; the classifying space of a topological group such as $B S^{1}$; the classifying space of finite groups in modular characteristics.) However, if the unit $\mathcal{O}_{X} \in \mathrm{QC}(X)$ is itself compact, then all dualizable objects are compact, since Hom from a dualizable object $M$ is the composition of the colimit-preserving functors internal $\operatorname{Hom} \mathcal{H o m}(M,-)$ and global sections $\Gamma(X,-)$.

Lemma 3.5 ([BoN, 6.4], [EKMM, III.7.9], [L4, 4.7.2]). For the $\infty$-category $\operatorname{Mod}_{k}=$ $\mathrm{QC}(\operatorname{Spec} k)$ of modules over a commutative derived ring (that is, quasi-coherent sheaves on an affine derived scheme), all three notions of finiteness coincide: $M$ compact $\Longleftrightarrow M$ dualizable $\Longleftrightarrow M$ perfect.

Proof. First, note that the free module $k$, which is the monoidal unit, is clearly compact. Hence all dualizable objects are compact. Moreover, we can write any object as a colimit of free modules. For $M$ compact, the identity map $\operatorname{id}_{M} \in$ $\operatorname{Hom}(M, M)$ has to factor through a finite colimit, showing that $M$ is perfect. Finally, perfect modules are dualizable since we can explicitly exhibit their dual as a finite limit of free modules.

It is useful to note that the notion of dualizable is local. On the one hand, pullback for any map of stacks (for example, restriction to an affine map) preserves dualizable objects. On the other hand, a dual object with its unit and trace maps is functorially characterized; thus if it exists locally, it will glue together to a global object. This observation leads to the identification of perfect and dualizable objects in $\mathrm{QC}(X)$ for any $X$ :

Proposition 3.6. For a derived stack $X$, an object of $\mathrm{QC}(X)$ is dualizable if and only if it is perfect.

Proof. Let $M \in \mathrm{QC}(X)$ be dualizable with dual $M^{\vee}$. Then for any map $\eta$ : Spec $A \rightarrow X$, the pullback $\eta^{*} M$ is dualizable with dual $\eta^{*} M^{\vee}$. Dualizable objects of $\operatorname{Mod}_{A}$ are perfect; hence $\eta^{*} M$ is perfect and so by definition, $M$ is perfect.

Now suppose $M \in \mathrm{QC}(X)$ is perfect. Recall that by definition, we have

$$
\mathrm{QC}(X) \simeq \lim _{\operatorname{Spec} A \in A f f / X} \operatorname{Mod}_{A} .
$$


Since $M$ is perfect, for any map $\eta: \operatorname{Spec} A \rightarrow X$, the pullback $\eta^{*} M$ is perfect, hence dualizable. We take the value of the dual $M^{\vee}$ along a map $\eta$ : Spec $A \rightarrow X$ to be the dual of the pullback $\left(\eta^{*} M\right)^{\vee}$. Note that $M^{\vee}$ is well-defined, since for any composite $\eta \circ \nu: \operatorname{Spec} B \rightarrow X$, there is a natural equivalence $\left(\nu^{*} \eta^{*} M\right)^{\vee} \simeq \nu^{*}\left(\left(\eta^{*} M\right)^{\vee}\right)$.

To exhibit $M$ and $M^{\vee}$ as dual to one another, we must construct the requisite unit and counit maps $u: \mathcal{O}_{X} \rightarrow M \otimes M^{\vee}$ and $c: M^{\vee} \otimes M \rightarrow \mathcal{O}_{X}$. Again using the definition of $\mathrm{QC}(X)$ as a limit, to produce one of these maps, it suffices to define analogous maps for the pullbacks under each $\eta: \operatorname{Spec} A \rightarrow X$ which themselves are compatible under pullbacks. But the existence of such maps is an immediate consequence of the definition $\eta^{*} M^{\vee}=\left(\eta^{*} M\right)^{\vee}$. Finally, to verify that the usual compositions $M \rightarrow M \otimes M^{\vee} \otimes M \rightarrow M$ and $M^{\vee} \rightarrow M^{\vee} \otimes M \otimes M^{\vee} \rightarrow M^{\vee}$ are equivalences, it suffices to check under pullbacks to affine maps. But this is a direct consequence of our definition of $M^{\vee}$ and the fact that pullbacks preserve tensor products.

3.1.2. Generators. Now we review notions of what it means for compact objects to generate a stable $\infty$-category. (See [L3, 17] for more details, and [L2, 5.5.7] for the general setting of presentable $\infty$-categories.)

Definition 3.7. A stable category $\mathcal{C}$ is said to be compactly generated if there is a small $\infty$-category $\mathcal{C}^{\circ}$ of compact objects $C_{i} \in \mathcal{C}$ whose right orthogonal vanishes: if $M \in \mathcal{C}$ satisfies $\operatorname{Hom}_{\mathcal{C}}\left(C_{i}, M\right) \simeq 0$, for all $i$, then $M \simeq 0$.

As explained in [L3, Remark 17.3], whether a stable $\infty$-category is compactly generated can be studied completely in the underlying homotopy category. In particular, the notion for stable $\infty$-categories is compatible with that for triangulated categories.

Example 3.8. For a commutative derived ring $k$, the stable $\infty$-category $\operatorname{Mod}_{k}$ of $k$-modules is compactly generated. In fact, it is generated by the free module $k$ itself.

On the one hand, for a stable small $\infty$-category $\mathcal{C}^{\circ}$, the inductive limit $\mathcal{C}=$ $\operatorname{Ind}\left(\mathcal{C}^{\circ}\right)$ is a compactly generated stable presentable $\infty$-category. Furthermore (see [L2, 5.3.4]), if $\mathcal{C}^{\circ}$ is closed under finite colimits and idempotent complete, then it can be recovered as the compact objects of $\mathcal{C}$. In particular, we have that $\mathcal{C}$ is the Ind-category of its compact objects $\mathcal{C}^{\circ}$.

On the other hand, given a stable $\infty$-category $\mathcal{C}$ with a small full $\infty$-subcategory $\mathcal{C}^{\circ}$ of compact generators, one can recover all compact objects of $\mathcal{C}$ by a result of Neeman [N1] (see also [L2, Proposition 5.3.4.17]): the compact objects are precisely direct summands of the objects of the smallest stable $\infty$-subcategory $\mathcal{C}_{s}$ containing $\mathcal{C}^{\circ}$ (that is, they are direct summands of finite iterated extensions of objects of $\mathcal{C}^{\circ}$ ). In particular, if $\mathcal{C}^{\circ}$ is stable and idempotent complete, then it consists precisely of the compact objects of $\mathcal{C}$.

If we further assume that $\mathcal{C}$ is a presentable stable $\infty$-category $\mathcal{C}$ with a small full $\infty$-subcategory $\mathcal{C}^{\circ}$ of compact generators, then a theorem of Schwede and Shipley SSh guarantees that we can recover $\mathcal{C}$ as the cocompletion of $\mathcal{C}_{s}$ (see [L4, 4.4] for the $\infty$-categorical version and $[\mathrm{Ke}$ for the differential graded version). In other words, we recover $\mathcal{C}$ by passing to the category of colimit-preserving $k$-linear functors to $k$-modules

$$
\mathcal{C} \simeq \operatorname{Fun}\left(\mathcal{C}_{s}^{\text {op }}, \operatorname{Mod}_{k}\right)
$$


In particular, we now can check that our notion of a perfect stack is equivalent to more familiar assumptions on a symmetric monoidal $\infty$-category.

Proposition 3.9. For a derived stack $X$ with affine diagonal, the following are equivalent:

(1) $X$ is perfect.

(2) $\mathrm{QC}(X)$ is compactly generated, and its compact and dualizable objects coincide.

Proof. If $X$ is perfect, so that $\mathrm{QC}(X)=\operatorname{Ind} \operatorname{Perf}(X)$, we claim that compact and dualizable objects agree, and hence generate compact objects, so that (1) implies (2).

To see the claim, it suffices to show that the full $\infty$-subcategory of dualizable objects of $\mathrm{QC}(X)$ is idempotent complete (since it is also stable). Since $\mathrm{QC}(X)$ is idempotent complete, this is equivalent to showing that dualizable objects are closed under retracts. However, for a retract $N$ of a dualizable object $M$ one can explicitly write down the unit and trace maps for $N \otimes \mathcal{H o m}\left(N, \mathcal{O}_{X}\right)$ and confirm the necessary conditions. We leave this to the reader.

Conversely, if $\mathrm{QC}(X)$ is compactly generated, then $\mathrm{QC}(X)$ is the Ind-category of its compact objects, and hence by assumption also the Ind-category of its dualizable objects, so that (2) implies (1).

3.2. Base change and the projection formula. In this section, we collect properties of the pushforward along a perfect stack, as summarized in the following proposition:

Proposition 3.10. Let $f: X \rightarrow Y$ be a perfect morphism. Then $f_{*}: \mathrm{QC}(X) \rightarrow$ $\mathrm{QC}(Y)$ commutes with all small colimits and satisfies the projection formula. Furthermore, if $g: Y^{\prime} \rightarrow Y$ is any map of derived stacks, the resulting base change map $g^{*} f_{*} \rightarrow f_{*}^{\prime} g^{\prime *}$ is an equivalence.

Remark 3.11. The conclusions of the proposition in fact do not require the full strength of the assumption that $f$ be perfect. The only hypothesis needed is that $f_{*}$ preserve colimits, or equivalently that the unit is relatively compact.

Remark 3.12. In the setting of simplicial commutative rings, and for $f: X \rightarrow Y$ a bounded, separated, quasi-compact relative derived algebraic space, the following results can essentially be found in Proposition 5.5.5 of Lurie's thesis. The arguments in this section are an expanded version of parts of the arguments there, which extend largely unchanged to any reasonable setting for derived algebraic geometry such as $\mathcal{E}_{\infty}$-ring spectra.

Let us first consider the case when $Y=\operatorname{Spec} A$ is affine, so that $f: X \rightarrow \operatorname{Spec} A$ is perfect over $A$. Then the pushforward $f_{*}$ coincides with the global sections functor $\Gamma: \mathrm{QC}(X) \rightarrow \operatorname{Mod}_{A}$. Over an affine base, $f_{*}$ is colimit-preserving if and only if the structure sheaf $\mathcal{O}_{X}$ (which is always dualizable) is a compact object of $\mathrm{QC}(X)$, which follows from $f$ being perfect.

Lemma 3.13. Let $M \in \mathrm{QC}(X)$ and let $N \in \mathrm{QC}(Y) \simeq \operatorname{Mod}_{A}$. Then the natural projection map $f_{*} M \otimes N \rightarrow f_{*}\left(M \otimes f^{*} N\right)$ is an equivalence.

Proof. Tensor products and pullbacks always preserve colimits, and in our setting, $f_{*}$ is colimit-preserving as well. Therefore for any $M$ the functors $f_{*} M \otimes(-)$ and 
$f_{*}\left(M \otimes f^{*}-\right)$ define colimit-preserving endofunctors of $\operatorname{Mod}_{A}$. Hence both functors are determined by their value on $A$ and canonically take the value $f_{*} M$. We find that the natural map is an equivalence.

Let us continue with $f: X \rightarrow \operatorname{Spec} A$ as above, and consider $g: \operatorname{Spec} B \rightarrow \operatorname{Spec} A$ to be an arbitrary map of affine derived schemes. Consider the Cartesian square

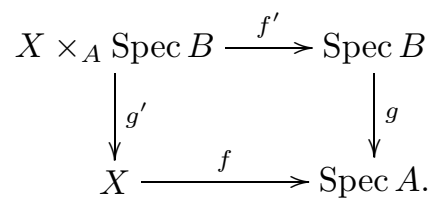

Lemma 3.14. The natural base change morphism $g^{*} f_{*} \rightarrow f_{*}^{\prime} g^{\prime *}$ is an equivalence.

Proof. Since $g$ is affine and hence $g^{\prime}$ is as well, the fiber product $X \times{ }_{A}$ Spec $B$ can be identified with the relative spectrum $\operatorname{Spec}_{X} C$ of a commutative algebra object $C \in$ $\operatorname{Alg}(\mathrm{QC}(X))$, and $g_{*}^{\prime}$ induces an equivalence $\mathrm{QC}\left(X \times{ }_{A} \operatorname{Spec} B\right) \simeq \operatorname{Mod}_{C}(\mathrm{QC}(X))$. Furthermore, the pullback $g^{\prime *}$ can be described as tensoring with $C$, and thus in particular $f_{*}^{\prime} g^{\prime *} M \simeq f_{*}(C \otimes M)$. However, the global sections functor takes fiber products to tensor products, so we can identify $C \simeq f^{*} g_{*} B$. Applying the previously established projection formula twice, we can now compute $f_{*}\left(f^{*} g_{*} B \otimes\right.$ $M) \simeq f_{*} M \otimes_{A} g_{*} B \simeq g^{*} f_{*} M \otimes_{B} B \simeq g^{*} f_{*} M$, completing the proof.

Now consider the general case where $f: X \rightarrow Y$ is any perfect morphism. Let us define a pushforward functor $f_{+}: \mathrm{QC}(X) \rightarrow \mathrm{QC}(Y)$ by requiring it to satisfy base change for affine derived schemes over $Y$. That is, for any $M \in \mathrm{QC}(X)$, let us define $f_{+} M$ to take the value $\left(f_{+} M\right)(g)=g_{*}^{\prime} f^{\prime *} M$, for any $g: \operatorname{Spec} B \rightarrow Y$. As a corollary of the previous lemma, we can verify that this definition is sensible.

Corollary 3.15. $f_{+} M$ is a well-defined quasi-coherent sheaf on $Y$.

Proof. Since $\mathrm{QC}(Y) \simeq \lim _{A f f} / Y \operatorname{Mod}_{B}$, the claim that $f_{+} M$ forms a quasi-coherent sheaf on $Y$ is equivalent to the claim that for any diagram of the form

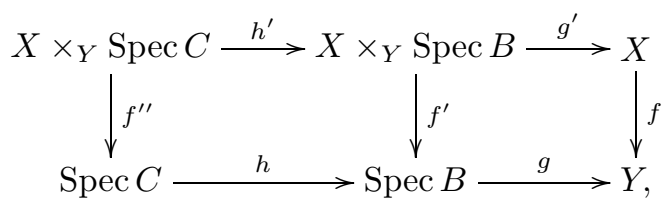

$f_{+} M(g \circ h)$ is canonically equivalent to $h^{*} f_{+} M(g)$. Unraveling these formulas, by definition we have that $f_{+} M(g \circ h)=f_{*}^{\prime \prime} h^{\prime *} g^{\prime *} M$ and that $h^{*} f_{+} M(g) \simeq h^{*} f_{*}^{\prime} g^{\prime *} M$. By the previous lemma, these are equivalent by base change in the left hand square: $f^{\prime}: X \times_{Y} \operatorname{Spec} B \rightarrow \operatorname{Spec} B$ is perfect, and so $h^{*} f_{*}^{\prime} \simeq f_{*}^{\prime \prime} h^{\prime *}$.

Lemma 3.16. The natural transformation $f_{*} \rightarrow f_{+}$is an equivalence of functors.

Proof. Take any $N \in \mathrm{QC}(Y)$ and $M \in \mathrm{QC}(X)$. First note that for any quasicoherent sheaf $K \in \mathrm{QC}(Z)$, there is an equivalence $K \simeq \lim _{g \in A f f / Z} g_{*} g^{*} K$. Thus we calculate

$$
\begin{aligned}
f_{+} M & \simeq \lim _{A f f / Y} g_{*} g^{*} f_{+} M \simeq \lim _{A f f} g_{*} f_{*}^{\prime} g^{\prime *} M \simeq \lim _{A f f / Y} g_{*} f_{*}^{\prime} g^{\prime *} M \\
& \simeq \lim _{A f f / Y} f_{*} g_{*}^{\prime} g^{\prime *} M \simeq f_{*}\left(\lim _{A f f / Y} g_{*}^{\prime} g^{\prime *} M\right) .
\end{aligned}
$$


To prove the lemma, it thus suffices to show that the natural map $M \rightarrow \lim g_{*}^{\prime} g^{\prime *} M$ is an equivalence.

This is a consequence of the stronger claim that the functor

$$
\mathrm{QC}(X) \rightarrow \lim _{A f f / Y} \mathrm{QC}\left(X \times_{Y} B\right)
$$

is an equivalence. Since the functor $\mathrm{QC}(-)$ takes all colimits of stacks to limits, it therefore suffices to show that the natural map $X \rightarrow \lim _{A f f / Y}\left(X \times_{Y} B\right)$ is an equivalence. This limit can be calculated by picking an affine cover $U \rightarrow Y$ and realizing $Y$ as the geometric realization of the usual simplicial object $U_{*} \rightarrow Y$. Finally, since geometric realization commutes with fiber products, we are done.

Since $f_{+}$was defined to satisfy base change and preserve colimits, we now have the following.

Proof of Proposition 3.10. The first assertions were proved above. Since base change is local in the target, one can prove the final statement for an arbitrary $Y^{\prime} \rightarrow Y$ by choosing a cover of $Y^{\prime}$ by an affine Spec $A \rightarrow Y^{\prime}$, thus reducing to the case which was proved above.

3.3. Constructions of perfect stacks. In this section, we construct many examples of perfect stacks.

Throughout what follows, by a derived scheme, we mean a derived stack which admits a Zariski open covering by affine derived schemes. Recall that a morphism is Zariski open if it induces a Zariski open morphism on the underlying truncated underived stacks, as well as isomorphisms of the higher homotopy groups of the structure sheaves over the Zariski open substack. Equivalently, one can think of a derived scheme in terms of the underlying truncated underived scheme equipped with a derived enhancement of the structure sheaf. Following the usual conventions, we say that a derived scheme is quasi-compact if any Zariski open cover admits a finite refinement.

We begin with two lemmas needed to show that quasi-compact derived schemes with affine diagonal are in fact perfect.

Lemma 3.17. For $X$ a quasi-compact derived scheme with affine diagonal the global sections functor $\Gamma: \mathrm{QC}(X) \rightarrow \operatorname{Mod}_{A}$ is colimit-preserving.

Proof. We briefly sketch the argument of [L1, Proposition 5.5.5]. The derived global sections functor $\Gamma$ preserves finite colimits. Thus it suffices to show that $\Gamma$ preserves small coproducts: we must check that the natural map $\coprod_{\alpha} \Gamma\left(M_{\alpha}\right) \rightarrow \Gamma\left(\coprod_{\alpha} M_{\alpha}\right)$ is an equivalence, i.e., that the induced map on homotopy groups $\coprod_{\alpha} \pi_{*} \Gamma\left(M_{\alpha}\right) \rightarrow$ $\pi_{*} \Gamma\left(\coprod_{\alpha} M_{\alpha}\right)$ is an equivalence. Since $\pi_{*}$ is colimit-preserving, it suffices to check that the individual terms $\pi_{i} \Gamma$ each preserve small coproducts.

This is shown in two steps. First, one checks that for $M \in \mathrm{QC}(X)$ concentrated in a single degree, there exists $m$ such that $\pi_{n} \Gamma M$ is zero for all $n<m$. Thus to establish the assertion, it suffices to work in the subcategory $M \in(\mathrm{QC}(X)){ }_{\geq n}^{\leq n+m}$ for which $\pi_{*} \Gamma M$ is concentrated in bounded degrees.

Next, one chooses a finite affine cover $U \rightarrow X$ giving the usual simplicial object $U_{*} \rightarrow X$, and thus an identification $\Gamma M \simeq \lim \left(\left.M\right|_{U_{q}}\right)$. The resulting Bousfield-Kan, or Cech, spectral sequence has $E_{1}$-term given by the $\pi_{p}\left(\left.M\right|_{U_{q}}\right)$ and converges to $\pi_{*} \Gamma M$. (The construction of the $E_{1}$-term evidently commutes with coproducts in $\mathrm{QC}(X)$, since pullback to an affine map is colimit-preserving.) Using the previous 
step, only finitely many terms in the spectral sequence may involve differentials affecting a particular group $\pi_{i} \Gamma M$. Therefore we have expressed $\pi_{i} \Gamma M$ as a finite limit of terms which preserved colimits in $M$, and so the result follows.

The following shows that we can glue derived schemes with finite colimits rather than geometric realizations. We state it in the simplest form applicable to the assertions which follow.

Lemma 3.18. Suppose $X$ is a derived scheme, and $U \amalg V \rightarrow X$ is an open Zariski cover. Then the following is a colimit diagram:

$$
U \cap V \Longrightarrow U \amalg V \longrightarrow X .
$$

Proof. It suffices to show that the following diagram of algebra objects is Cartesian:

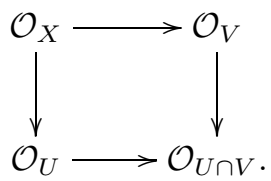

Let $u: U \amalg V \rightarrow X$ denote the cover. Since the restriction $u^{*}(-) \simeq(-) \otimes_{\mathcal{O}_{X}}$ $\left(\mathcal{O}_{U} \times \mathcal{O}_{V}\right)$ is conservative and preserves finite limits, it suffices to show that the restriction of the above diagram is Cartesian. But this is nothing more than the clearly Cartesian diagram

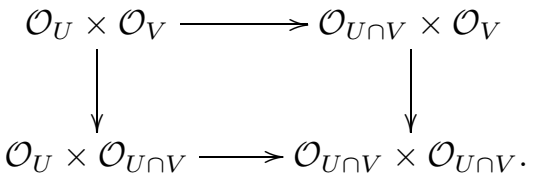

Proposition 3.19. Quasi-compact derived schemes with affine diagonal are perfect.

Proof. The result for ordinary (nonderived) schemes $X$ is a theorem of Neeman [N2, extending ideas of Thomason [TT. (In fact, Neeman proves that for quasicompact, quasi-separated schemes, $\mathrm{QC}(X)$ is compactly generated, and dualizable and compact objects coincide. We assume $X$ has affine diagonal only because the definition of perfect stack requires it.)

A modified exposition of Neeman's argument appears in the work of Bondal and Van den Bergh [BV], who in fact prove that $\mathrm{QC}(X)$ is generated by a single perfect object. One can translate the latter proof, which occupies [BV, Section 3.3], directly into the derived setting, substituting Lemma 3.17 above for its underived version BV, Corollary 3.3.4], and using the natural identification $\mathrm{QC}(\operatorname{Spec} A) \simeq \operatorname{Mod}_{k}$ instead of [BV] Corollary 3.3.5]. (In the derived setting, there is no general notion of the abelian category of quasi-coherent sheaves, so we do not need to worry about the potential distinction between its derived category and the quasi-coherent derived category.) In what follows, we sketch the argument for the reader's convenience, keeping the notation from [BV].

The proof that $\mathrm{QC}(X)$ is generated by a single perfect (dualizable) object is an induction on the number of open sets in an affine cover of $X$. The base case of an affine derived scheme is Lemma3.5. For the inductive step, we write $X=Y \cup U$ with $Y$ open and $U$ affine (putting us in the context of Lemma 3.18), and assume that 
$\mathrm{QC}(Y)$ has a perfect generator E. By [BoN, Proposition 6.1], there is an explicit compact generator $Q$ for the kernel of the restriction from $U$ to the intersection $S=Y \cap U$. (One can think of $Q$ as a form of the structure sheaf of the closed complement $V=U \backslash S$.) The key to the inductive step is Neeman's abstract categorical form [N1, Theorem 2.1] of Thomason's extension theorem for compact objects. This allows us to extend $\left.E \oplus E[1]\right|_{S}$ to a compact (hence perfect) object on $U$, and then to glue the latter to $E \oplus E[1]$ to obtain a perfect object $P$ on all of $X$. (Note that we extend $E \oplus E[1]$ rather than $E$ itself since $K$-theoretic obstructions vanish for the former.) One then checks by a Mayer-Vietoris argument that the sum of $P$ and the pushforward of $Q$ (which is itself compact and perfect by support considerations) to $X$ generates all of $\mathrm{QC}(X)$.

By Lemma 3.17, we know that $\mathcal{O}_{X}$ is compact, and hence that dualizable complexes are compact. The assertion that compact objects are dualizable follows from [N1]: if a set $\mathcal{C}^{\circ}$ of compact objects generates $\mathcal{C}$, then all compact objects of $\mathcal{C}$ are summands of finite colimits of objects of $\mathcal{C}^{\circ}$ and their shifts. Since $\mathrm{QC}(X)$ is generated by a perfect object, we conclude that all compact objects are summands of perfect objects, which allows one to check locally that they are indeed perfect.

We next make a simple observation about compact objects.

Lemma 3.20. Suppose $p: X \rightarrow Y$ is a perfect morphism over a perfect base. Then compact and dualizable objects of $\mathrm{QC}(X)$ coincide.

Proof. First note that the pushforward $p_{*}$ along the perfect morphism $p$ is colimitpreserving; hence (by adjunction) the pullback $p^{*} M$ of a compact object $M \in$ $\mathrm{QC}(Y)$ is compact. In particular we find that the structure sheaf (the monoidal unit) on $X$ is compact, and hence that all dualizable objects are compact. Note also that the pullback of a dualizable object is always dualizable.

Now suppose that $U \rightarrow Y$ is any affine mapping to $Y$ and consider the base change $X_{U}$ of $X$ to $U$. By the definition of a perfect morphism applied to $p$, this base change is itself a perfect stack. Let $q: X_{U} \rightarrow X$ denote the base change morphism, which is affine since $Y$ has affine diagonal. If $M \in \mathrm{QC}(X)$ is any compact object and $M_{U}=q^{*} M$ is its pullback to $U$, then $M_{U}$ is itself compact since $q_{*}$ preserves colimits:

$$
\begin{aligned}
\operatorname{Hom}\left(M_{U}, \operatorname{colim} A_{i}\right) & \simeq \operatorname{Hom}\left(q^{*} M, \operatorname{colim} A_{i}\right) \\
& \simeq \operatorname{Hom}\left(M, q_{*} \operatorname{colim} A_{i}\right) \\
& \simeq \operatorname{Hom}\left(M, \operatorname{colim} q_{*} A_{i}\right) \\
& \simeq \operatorname{colim} \operatorname{Hom}\left(M, q_{*} A_{i}\right) \\
& \simeq \operatorname{colim} \operatorname{Hom}\left(M_{U}, A_{i}\right) .
\end{aligned}
$$

Since $U$ itself is perfect, it follows that $M_{U}$ is dualizable and (by Proposition 3.6) perfect. We now show that the pullback of $M$ to any affine map is perfect. Assume that the map $U \rightarrow Y$ is surjective, so as a consequence $X_{U} \rightarrow X$ is also surjective. Now let $f: V \rightarrow X$ be any affine mapping to $X$. We may form the Cartesian diagrams:

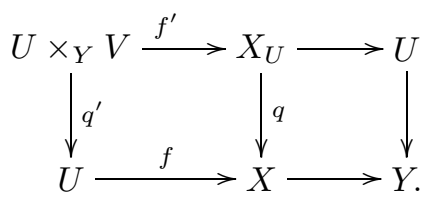


We now verify that $f^{*} M$ is perfect, given the hypotheses above. The fiber product $U \times_{Y} V$ is affine, since $Y$ has affine diagonal, and the map $q^{\prime}$ is surjective since $q$ is. Since $M_{U}$ is perfect, $f^{\prime *} M_{U} \simeq q^{\prime *} f^{*} M$ is perfect, as well. Thus, in summary, we know that $q^{* *} f^{*} M=\mathcal{O}\left(V \times_{Y} U\right) \otimes_{\mathcal{O}(U)} f^{*} M$ is perfect, where $q^{\prime}$ is surjective, and hence $f^{*} M$ is perfect. Since the pullback of $M$ to any affine map is perfect, therefore $M$ is itself perfect by Definition 3.1 and hence (again by Proposition 3.6) dualizable.

Proposition 3.21. Let $Y$ be a perfect stack and $p: X \rightarrow Y$ a relative quasicompact derived scheme with affine diagonal and a relatively ample family of line bundles (for example, $p$ quasi-projective, or in particular affine). Then $X$ is perfect.

Proof. By Lemma 3.20, we know that compact and dualizable objects in $\mathrm{QC}(X)$ coincide. Thus we need only to check that $\mathrm{QC}(X)$ is compactly generated. The pullbacks $p^{*} M$ of compact (hence dualizable) objects on $Y$ are dualizable, hence compact, as are the line bundles $\mathcal{L}$ in the given relatively ample family. We claim that the $\infty$-category of compact objects $p^{*} M \otimes \mathcal{L}$ generates $\mathrm{QC}(X)$. The argument is as above in the case of an external product: let $N$ be right orthogonal to the compact objects, so that $\operatorname{Hom}\left(p^{*} M \otimes \mathcal{L}, N\right)=0$. We first find by adjunction and the fact that $Y$ is compactly generated that $p_{*} \mathcal{H} \operatorname{om}(\mathcal{L}, N)=0$. Since the objects $\mathcal{L}$ form a relatively ample family of line bundles this forces $N=0$.

Corollary 3.22. In characteristic zero, the quotient $X / G$ of a quasi-projective derived scheme $X$ by a linear action of an affine algebraic group $G$ is perfect.

Proof. First note that in characteristic zero, $B G L_{n}$ is clearly perfect: the compact and dualizable objects are both finite-dimensional representations, which generate $\mathrm{QC}\left(B G L_{n}\right)$.

If $G$ is an affine algebraic group, we can embed $G \hookrightarrow G L_{n}$ as a subgroup of $G L_{n}$ for some $n$. Thus we obtain a morphism $B G \rightarrow B G L_{n}$ with fiber $G L_{n} / G$. By a theorem of Chevalley $\mathrm{Ch}, G L_{n} / G$ is a quasi-projective variety, and so by Proposition 3.21, $B G$ itself is perfect.

Finally, for a quasi-projective derived scheme $X$ with a linear action of $G$, the morphism $X / G \rightarrow B G$ is quasi-projective, so applying Proposition 3.21 again, we conclude that $X / G$ is perfect.

Corollary 3.23. Let $p: X \rightarrow Y$ be a morphism between perfect stacks. Then $p$ is perfect.

Proof. Let $f: A \rightarrow Y$ be a morphism from an affine derived scheme to the base, and $X_{A} \rightarrow A$ the base change of $X$. Since $Y$ has affine diagonal, $f$ is affine as is the base change morphism $f_{X}: X_{A} \rightarrow X$. In particular, Proposition 3.21 (again in the basic case of an affine morphism) applies to $f_{X}$, so that the total space $X_{A}$ is perfect.

Proposition 3.24. The product $X=X_{1} \times X_{2}$ of perfect stacks is perfect. More generally, for maps $p_{i}: X_{i} \rightarrow Y$, if $Y$ has affine diagonal, then $X_{1} \times_{Y} X_{2}$ is perfect.

Proof. The second assertion follows from the first and Proposition 3.21] since $X_{1} \times_{Y}$ $X_{2} \rightarrow X_{1} \times X_{2}$ is an affine morphism for $Y$ with affine diagonal.

To prove the first assertion, note that $X=X_{1} \times X_{2}$ has affine diagonal. Applying Lemma 3.20 to the projection to a factor, we see that compact and dualizable 
objects of $\mathrm{QC}(X)$ coincide. Thus to confirm that $X$ is perfect, it suffices to show that $\mathrm{QC}(X)$ is compactly generated.

Let us first check that the external product of compact objects is again compact. By assumption, compact objects $M_{i}$ on the factors $X_{i}$ are dualizable, so $M_{1} \otimes M_{2}$ is dualizable (it is the tensor product of pullbacks, and both operations preserve dualizabie objects) and hence compact.

Now let us check that external products of compact objects generate $\mathrm{QC}(X)$. The argument is a modification of the argument of Bondal and Van den Bergh BV] in the case of a single compact generator. Namely, let $N$ be right orthogonal to $\mathrm{QC}(X)^{c}$, so that in particular $\operatorname{Hom}\left(M_{1} \otimes M_{2}, N\right) \simeq 0$ for all $M_{i} \in \mathrm{QC}\left(X_{i}\right)^{c}$. By adjunction, we have

$$
\begin{aligned}
0 & \simeq \operatorname{Hom}\left(M_{1} \otimes M_{2}, N\right) \\
& \simeq \operatorname{Hom}\left(\pi_{1}^{*} M_{1}, \mathcal{H o m}\left(\pi_{2}^{*} M_{2}, N\right)\right) \\
& \simeq \operatorname{Hom}\left(M_{1}, \pi_{1, *} \mathcal{H o m}\left(\pi_{2}^{*} M_{2}, N\right)\right)
\end{aligned}
$$

for all $M_{1}, M_{2}$, so that $\pi_{1 *} \mathcal{H}$ om $\left(\pi_{2}^{*} M_{2}, N\right) \simeq 0$ since such an $M_{1}$ generates $\mathrm{QC}\left(X_{1}\right)$. For any affine maps $U \rightarrow X_{1}$ and $V \rightarrow X_{2}$, we therefore have

$$
\begin{aligned}
0 & \simeq \Gamma\left(U, \pi_{1, *} \mathcal{H o m}\left(\pi_{2}^{*} M_{2}, N\right)\right) \\
& \simeq \operatorname{Hom}_{U \times X_{2}}\left(\pi_{2}^{*} M_{2}, N\right) \\
& \simeq \operatorname{Hom}_{X_{2}}\left(M_{2},\left(\left.\pi_{2}\right|_{U \times X_{2}}\right)_{*} N\right)
\end{aligned}
$$

for all $M_{2}$. Since the latter objects generate $\mathrm{QC}\left(X_{2}\right)$, it follows (upon restricting to $V$ ) that $\Gamma(U \times V, N) \simeq 0$, whence (by affineness of $U \times V)$ that $\left.N\right|_{U \times V} \simeq 0$, and finally (since affine maps of the form $U \times V$ cover $X_{1} \times X_{2}$ ) that $N \simeq 0$.

Corollary 3.25. Let $X$ be a perfect stack and let $\Sigma$ be a finite simplicial set. Then the mapping stack $X^{\Sigma}=\operatorname{Map}(\Sigma, X)$ is perfect.

Proof. Let $\Sigma_{0}$ be the 0 -simplices of $\Sigma$. Since $X$ has affine diagonal, the natural projection $X^{\Sigma} \rightarrow X^{\Sigma_{0}}$ is affine. By Proposition 3.24, the product $X^{\Sigma_{0}}$ is perfect, and so by Proposition 3.21 (in the basic case of an affine morphism), the assertion follows.

Finally, we consider arbitrary quotients by finite group schemes in good characteristics.

Proposition 3.26. Let $X$ be a perfect stack with an action of an affine group scheme $G$ for which

(1) the global functions $\Gamma\left(G, \mathcal{O}_{G}\right)$ are a perfect complex;

(2) the unit $\mathcal{O}_{B G}$ on $B G$ is compact (equivalently, the trivial $G$-module is perfect).

Then $X / G$ is perfect.

Proof. We leave to the reader the exercise of checking that $X / G$ has affine diagonal since $X$ has affine diagonal and $G$ is affine.

We will first prove that condition (1) implies that $\mathrm{QC}(X / G)$ is generated by compact dualizable objects. Since $f: X \rightarrow X / G$ is affine, we have the identification $\mathrm{QC}(X) \simeq \operatorname{Mod}_{f_{*} \mathcal{O}_{X}}(\mathrm{QC}(X / G))$. 
We claim that the algebra object $f_{*} \mathcal{O}_{X}$ is perfect (or equivalently, dualizable). To see this, consider the pullback square of derived stacks

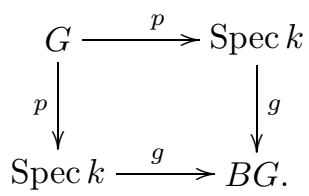

Via base change, we obtain an equivalence $g^{*} g_{*} \mathcal{O}_{k} \simeq p_{*} p^{*} \mathcal{O}_{k}$, or in other words, an equivalence of $k$-algebras $g^{*} g_{*} \mathcal{O}_{k} \simeq \Gamma\left(G, \mathcal{O}_{G}\right)$. By assumption, $\Gamma\left(G, \mathcal{O}_{G}\right)$ is a perfect complex, and $g^{*}$ is conservative and preserves perfect complexes, so we conclude that the pushforward $g_{*} \mathcal{O}_{k}$ is perfect. Now consider the pullback square of derived stacks

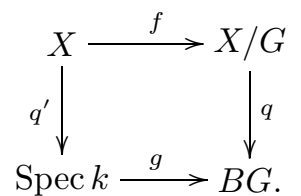

Since $g_{*} \mathcal{O}_{k}$ is perfect, $q^{*} g_{*} \mathcal{O}_{k}$ is perfect. By base change, we have the equivalence $q^{*} g_{*} \mathcal{O}_{k} \simeq f_{*} q^{\prime *} \mathcal{O}_{k}$, and thus we conclude that $f_{*} \mathcal{O}_{X}$ is perfect.

Next observe that the right adjoint $f^{+}$to the pushforward $f_{*}$ can be calculated explicitly by

$$
f^{+}(M) \simeq \mathcal{H o m}_{\mathcal{O}_{X / G}}\left(f_{*} \mathcal{O}_{X}, M\right) \simeq M \otimes_{\mathcal{O}_{X / G}}\left(f_{*} \mathcal{O}_{X}\right)^{\vee} .
$$

It follows immediately that $f^{+}$preserves colimits. It also follows that $f^{+}$is conservative since a diagram chase with the above identities leads to the identity

$$
\begin{aligned}
f^{*}\left(M \otimes_{\mathcal{O}_{X / G}}\left(f_{*} \mathcal{O}_{X}\right)^{\vee}\right) & \simeq f^{*}(M) \otimes_{\mathcal{O}_{X}} q^{\prime *} g^{*}\left(\left(g_{*} \mathcal{O}_{k}\right)^{\vee}\right) \\
& \simeq f^{*}(M) \otimes_{\mathcal{O}_{X}}\left(\mathcal{O}_{X} \otimes_{\mathcal{O}_{k}} \Gamma\left(G, \mathcal{O}_{G}\right)^{\vee}\right) .
\end{aligned}
$$

The unit $e: \operatorname{Spec} k \rightarrow G$ gives a factorization of the identity map

$$
\mathcal{O}_{k} \stackrel{p^{*}}{\longrightarrow} \Gamma\left(G, \mathcal{O}_{G}\right) \stackrel{e^{*}}{\longrightarrow} \mathcal{O}_{k},
$$

and taking duals, a factorization of the identity map of $\mathcal{O}_{k}$ through the dual $\Gamma\left(G, \mathcal{O}_{G}\right)^{\vee}$. Hence if $f^{+}(M)$ were trivial, then $f^{*}(M)$ would also be trivial, but $f^{*}$ is conservative. Thus we conclude that $f_{*}$ takes a generating set of compact objects to a generating set of compact objects.

We now appeal to condition (2) that the unit in $\mathrm{QC}(B G)$ is compact, hence so are all dualizables in $\mathrm{QC}(B G)$. In the case when $X$ is a point, the above arguments show that $\mathrm{QC}(B G)$ is compactly generated. Furthermore, it shows that all compacts are in fact dualizable (since $f_{*} \mathcal{O}_{k}$ is a compact dualizable generator), and hence $B G$ itself is perfect. The morphism $X / G \rightarrow B G$ is then a perfect morphism with perfect base. Thus by Lemma 3.20, compact and dualizable objects in $\mathrm{QC}(X / G)$ coincide. This implies (in combination with the compact generation of $\mathrm{QC}(X / G)$ above) that $X / G$ is perfect, as asserted.

\section{TENSOR PRODUCTS AND INTEGRAL TRANSFORMS}

In this section, we study the relation between the geometry and algebra of perfect stacks. We begin with some basic properties of tensor products of $\infty$-categories. Then we prove (Theorem 4.7) that the $\infty$-category of sheaves on a product of perfect 
stacks, and more generally a derived fiber product, is given by the tensor product of the $\infty$-categories of sheaves on the factors. This implies that the $\infty$-category of sheaves on a perfect stack is self-dual, which in turn allows us to describe $\infty$ categories of functors by $\infty$-categories of integral kernels (Corollary 4.10). Finally, we extend this last result to a relative setting where the base is an arbitrary derived stack with affine diagonal.

4.1. Tensor products of $\infty$-categories. In this section, we consider some properties of tensor products of $\infty$-categories that will be used in what follows. First we prove (Proposition 4.1) that $\infty$-categories of modules over associative algebra objects are dualizable, with duals given by modules for the opposite algebra, and that the tensor product of algebras induces tensor products on module categories. We then discuss the tensor product of small stable categories and its compatibility with passing to the corresponding presentable stable $\infty$-categories of Ind-objects.

4.1.1. Algebras and modules. Recall the tensor product of presentable stable $\infty$ categories developed in [L4], L5]. Namely, the $\infty$-category $\mathcal{P} r^{\mathrm{L}}$ of presentable $\infty$-categories (with morphisms given by left adjoints) carries a natural symmetric monoidal tensor product that preserves stable objects.

Let $\mathcal{C}$ be a monoidal $\infty$-category. For two presentable $\infty$-categories $\mathcal{M}$ and $\mathcal{M}^{\prime}$ left tensored over $\mathcal{C}$, we denote by $\operatorname{Fun}_{\mathcal{C}}^{\mathrm{L}}\left(\mathcal{M}, \mathcal{M}^{\prime}\right)$ the $\infty$-category of left adjoints from $\mathcal{M}$ to $\mathcal{M}^{\prime}$ that preserve the tensor over $\mathcal{C}$.

Similarly, the opposite category of $\mathcal{P} r^{\mathrm{L}}$ is the $\infty$-category $\mathcal{P} r^{\mathrm{R}}$ of presentable $\infty$-categories with morphisms given by right adjoints. For two presentable $\infty$ categories $\mathcal{M}$ and $\mathcal{M}^{\prime}$ left cotensored over $\mathcal{C}$, we denote by $\operatorname{Fun}_{\mathcal{C}}^{\mathrm{R}}\left(\mathcal{M}, \mathcal{M}^{\prime}\right)$ the $\infty$-category of right adjoints from $\mathcal{M}$ to $\mathcal{M}^{\prime}$ that preserve the cotensor over $\mathcal{C}$.

Proposition 4.1. Let $\mathcal{C}$ be a stable presentable symmetric monoidal $\infty$-category, and $A \in \mathcal{C}$ an associative algebra object.

(1) For any $\mathcal{C}$-module $\mathcal{M}$, there is a canonical equivalence of $\infty$-categories

$$
\operatorname{Mod}_{A}(\mathcal{C}) \otimes_{\mathcal{C}} \mathcal{M} \simeq \operatorname{Mod}_{A}(\mathcal{M}) .
$$

(2) For $A^{\prime} \in \mathcal{C}$, a second associative algebra, there is a canonical equivalence of $\infty$-categories

$$
\operatorname{Mod}_{A \otimes A^{\prime}}(\mathcal{C}) \simeq \operatorname{Mod}_{A}(\mathcal{C}) \otimes_{\mathcal{C}} \operatorname{Mod}_{A^{\prime}}(\mathcal{C}) .
$$

(3) The $\infty$-category of modules $\operatorname{Mod}_{A}(\mathcal{C})$ is dualizable as a $\mathcal{C}$-module with dual given by the $\infty$-category of modules $\operatorname{Mod}_{A^{\text {op }}}(\mathcal{C})$ over the opposite algebra.

The proof will depend on the following two lemmas:

Lemma 4.2. Let $\mathcal{M}$ and $\mathcal{M}^{\prime}$ be stable presentable $\infty$-categories that are left tensored and cotensored over $\mathcal{C}$. Let $G: \mathcal{M}^{\prime} \rightarrow \mathcal{M}$ be a right adjoint that is tensored and cotensored over $\mathcal{C}$. Assume further that $G$ is colimit-preserving. Then $G$ is conservative if the induced functor $\operatorname{Fun}_{\mathcal{C}}^{\mathrm{R}}\left(\mathcal{D}, \mathcal{M}^{\prime}\right) \rightarrow \operatorname{Fun}_{\mathcal{C}}^{\mathrm{R}}(\mathcal{D}, \mathcal{M})$ is conservative for any $\mathcal{D}$.

Proof. Suppose $G$ is not conservative. Then to prove the lemma, it suffices to exhibit a presentable $\infty$-category $\mathcal{D}$ also cotensored over $\mathcal{C}$ and a nontrivial right adjoint $j: \mathcal{D} \rightarrow \mathcal{M}^{\prime}$ cotensored over $\mathcal{C}$ such that $j \circ G$ is trivial. 
Define $\mathcal{D}$ to be the full $\infty$-subcategory of $\mathcal{M}^{\prime}$ of $G$-acyclic objects, that is, objects $m \in \mathcal{M}$ such that $G(m)$ is trivial. Our first task is to show that $\mathcal{D}$ is indeed presentable.

Observe that $\mathcal{D}$ is equivalent to the fiber product $\mathcal{D} \simeq 0 \times{ }_{\mathcal{M}} \mathcal{M}^{\prime}$, where the limit is computed in the $\infty$-category Cat $_{\infty}$ of $\infty$-categories. Recall by [L2, Proposition 5.5.3.13] that the natural functor $\mathcal{P} r^{\mathrm{L}} \rightarrow \mathrm{Cat}_{\infty}$ preserves limits. Furthermore, the forgetful functor $\operatorname{Mod}_{\mathcal{C}}\left(\mathcal{P} r^{\mathrm{L}}\right) \rightarrow \mathcal{P} r^{\mathrm{L}}$ also preserves limits since it has a left adjoint (given by induction).

Since the functor $G$ preserves colimits and is $\mathcal{C}$-linear, we may regard it as a morphism in $\operatorname{Mod}_{\mathcal{C}}\left(\mathcal{P} r^{\mathrm{L}}\right)$. Thus $\mathcal{D}$ can be computed as a limit in $\operatorname{Mod}_{\mathcal{C}}\left(\mathcal{P} r^{\mathrm{L}}\right)$, and so can be regarded as an object of $\mathcal{P} r^{\mathrm{L}}$. In other words, $\mathcal{D}$ is presentable and furthermore tensored over $\mathcal{C}$. Finally, since $\mathcal{D}$ is tensored over $\mathcal{C}$, it is automatically cotensored as well.

Now it remains to show that the inclusion $j: \mathcal{D} \rightarrow \mathcal{M}^{\prime}$ is indeed a right adjoint and cotensored over $\mathcal{C}$. Since $j$ preserves all limits and colimits (and in particular $\kappa$ filtered colimits), the adjoint functor theorem applies. Finally, since $G$ is cotensored over $\mathcal{C}, j$ is as well.

Lemma 4.3. Let $\mathcal{M}$ be a stable presentable $\infty$-category which is left tensored and cotensored over $\mathcal{C}$, and let $A$ be an associative algebra in $\mathcal{C}$. Then the forgetful functor $G: \operatorname{Mod}_{A}(\mathcal{C}) \otimes_{\mathcal{C}} \mathcal{M} \rightarrow \mathcal{M}$ is conservative.

Proof. Observe that for any $\mathcal{D}$ tensored over $\mathcal{C}$, the pullback

$$
\operatorname{Fun}_{\mathcal{C}}^{\mathrm{L}}\left(\operatorname{Mod}_{A}(\mathcal{C}) \otimes_{\mathcal{C}} \mathcal{M}, \mathcal{D}\right) \rightarrow \operatorname{Fun}_{\mathcal{C}}^{\mathrm{L}}(\mathcal{M}, \mathcal{D})
$$

induced by the induction $F: \mathcal{M} \rightarrow \operatorname{Mod}_{A} \otimes_{\mathcal{C}} \mathcal{M}$ is conservative. In other words, if a functor out of $\operatorname{Mod}_{A} \times \mathcal{M}$ (which preserves colimits in each variable) is trivial when restricted to $\mathcal{M}$, then it is necessarily trivial.

Consequently, switching to opposite categories, we have that the corresponding functor

$$
\operatorname{Fun}_{\mathcal{C}}^{\mathrm{R}}\left(\mathcal{D}, \operatorname{Mod}_{A} \otimes \mathcal{M}\right) \rightarrow \operatorname{Fun}_{\mathcal{C}}^{\mathrm{R}}(\mathcal{D}, \mathcal{M})
$$

induced by the forgetful functor $G: \operatorname{Mod}_{A} \otimes_{\mathcal{C}} \mathcal{M} \rightarrow \mathcal{M}$ is conservative.

Now we can apply Lemma 4.2 with $\mathcal{M}^{\prime}=\operatorname{Mod}_{A} \otimes \mathcal{M}$ to obtain that $G$ is conservative.

Proof of Proposition 4.1. We first prove that $\operatorname{Mod}_{A}(\mathcal{C}) \otimes_{\mathcal{C}} \mathcal{M}$ is equivalent to $\operatorname{Mod}_{A}(\mathcal{M})$ by the natural evaluation functor. Consider the adjunction

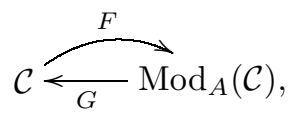

where $F(-)=A \otimes-$ is the induction and $G$ is the forgetful functor.

The above adjunction induces an adjunction

$$
\mathcal{M} \underset{G \otimes \mathrm{id}}{F \otimes \text { id }} \operatorname{Mod}_{A}(\mathcal{C}) \otimes_{\mathcal{C}} \mathcal{M} \longrightarrow \operatorname{Mod}_{T}(\mathcal{M})
$$

and thus a functor to modules over the monad $T=(G \otimes \mathrm{id}) \circ(F \otimes \mathrm{id})$ acting on $\mathcal{M}$. The functor underlying $T$ is given by tensoring with $A$, so we also have an equivalence $\operatorname{Mod}_{T}(\mathcal{M}) \simeq \operatorname{Mod}_{A}(\mathcal{M})$. 
By its universal characterization, the functor $G \otimes \mathrm{id}$ is colimit-preserving. Note as well that $G$ and hence $G \otimes$ id is also $\mathcal{C}$-linear (or in other words, the adjunction satisfies an analogue of the projection formula). Thus it follows from Lemma 4.3 that $G \otimes$ id is also conservative. Thus $G \otimes$ id satisfies the monadic Barr-Beck conditions, and we obtain the desired equivalence $\operatorname{Mod}_{A}(\mathcal{C}) \otimes_{\mathcal{C}} \mathcal{M} \simeq \operatorname{Mod}_{A}(\mathcal{M})$.

Next, we can apply this to the instance where $\mathcal{M}$ is the $\infty$-category of left modules over another associative algebra $A^{\prime}$ to conclude that there is a natural equivalence $\operatorname{Mod}_{A}(\mathcal{C}) \otimes_{\mathcal{C}} \operatorname{Mod}_{A^{\prime}}(\mathcal{C}) \simeq \operatorname{Mod}_{A}\left(\operatorname{Mod}_{A^{\prime}}(\mathcal{C})\right)$. We now have a chain of adjunctions

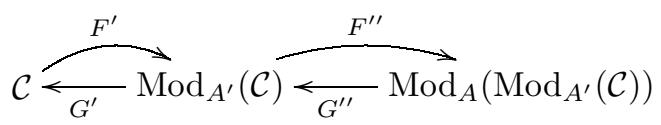

in which the composite $G^{\prime} \circ G^{\prime \prime}$ is colimit-preserving and conservative and hence satisfies the monadic Barr-Beck conditions.

Furthermore, the above adjunction naturally extends to a diagram in which the cycle of left adjoints (denoted by bowed arrows), and hence also the cycle of right adjoints (denoted by straight arrows), commute:

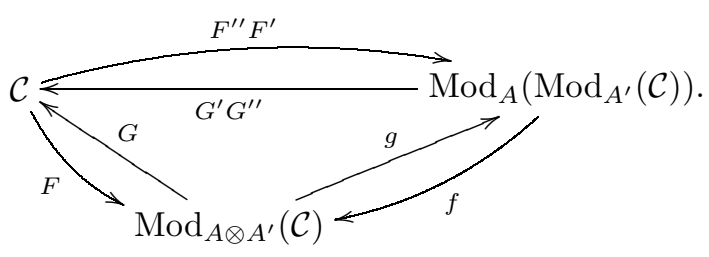

Here $F(-)=A_{1} \otimes A_{2} \otimes-$ is the induction, $G$ is the forgetful functor, $f$ is the natural functor factoring through $\operatorname{Mod}_{A}(\mathcal{C}) \otimes_{\mathcal{C}} \operatorname{Mod}_{A^{\prime}}(\mathcal{C})$, and $g$ is its right adjoint. From this diagram, we obtain a morphism of monads

$$
G^{\prime} G^{\prime \prime} F^{\prime \prime} F^{\prime} \longrightarrow G^{\prime} G^{\prime \prime} g f F^{\prime \prime} F^{\prime} \simeq G F .
$$

Now the underlying functors of the monads $G F(-)$ and $G^{\prime} G^{\prime \prime} F^{\prime \prime} F^{\prime}(-)$ are both equivalent to the tensor $A \otimes A^{\prime} \otimes(-)$, so the above morphism of monads is an equivalence. Thus we obtain the promised equivalence $\operatorname{Mod}_{A}(\mathcal{C}) \otimes_{\mathcal{C}} \operatorname{Mod}_{A^{\prime}}(\mathcal{C}) \simeq$ $\operatorname{Mod}_{A}\left(\operatorname{Mod}_{A^{\prime}}(\mathcal{C})\right) \simeq \operatorname{Mod}_{A \otimes A^{\prime}}(\mathcal{C})$.

Finally, we show that the $\infty$-category of left $A$-modules $\operatorname{Mod}_{A}(\mathcal{C})$ is a dualizable $\mathcal{C}$-module by directly exhibiting the $\infty$-category of right $A$-modules $\operatorname{Mod}_{A \text { op }}(\mathcal{C})$ as its dual. The trace map is given by the two-sided bar construction

$$
\tau: \operatorname{Mod}_{A}(\mathcal{C}) \otimes_{\mathcal{C}} \operatorname{Mod}_{A^{\text {op }}}(\mathcal{C}) \rightarrow \mathcal{C}, \quad M, N \mapsto M \otimes_{A} N
$$

The unit map is given by the induction

$$
u: \mathcal{C} \rightarrow \operatorname{Mod}_{A^{\mathrm{op}}}(\mathcal{C}) \otimes_{\mathcal{C}} \operatorname{Mod}_{A}(\mathcal{C}) \simeq \operatorname{Mod}_{A^{\mathrm{op}} \otimes A}(\mathcal{C}), \quad c \mapsto A \otimes c,
$$

where we regard $A \otimes c$ as an $A$-bimodule.

One can verify directly that the composition

$$
\operatorname{Mod}_{A}(\mathcal{C}) \stackrel{\operatorname{id} \otimes u}{\longrightarrow} \operatorname{Mod}_{A}(\mathcal{C}) \otimes_{\mathcal{C}} \operatorname{Mod}_{A} \text { op }(\mathcal{C}) \otimes_{\mathcal{C}} \operatorname{Mod}_{A}(\mathcal{C}) \stackrel{\tau \otimes \text { id }}{\longrightarrow} \operatorname{Mod}_{A}(\mathcal{C})
$$

is equivalent to the identity. First, $(\mathrm{id} \otimes u)(M)$ is equivalent to $A \otimes M$ regarded as an $A \otimes A^{\mathrm{op}} \otimes A$-module, and second, $(\tau \otimes \mathrm{id})(A \otimes M)$ is equivalent to $A \otimes_{A} M \simeq M$. 
4.1.2. Small stable categories. We have been working with the symmetric monoidal structure on the $\infty$-category $\mathcal{P} r^{\mathrm{L}}$ of presentable $\infty$-categories as developed in [L4], [L5]. We will also need the tensor product of small stable idempotent complete $\infty$ categories, in particular, the $\infty$-categories of compact objects in presentable stable $\infty$-categories.

Let $s t$ be the full $\infty$-subcategory of the $\infty$-category of stable categories (with morphisms exact functors) consisting of those $\infty$-categories that are idempotent complete. Recall that an $\infty$-category $\mathcal{C}$ is idempotent complete if the essential image of the Yoneda embedding $\mathcal{C} \rightarrow \mathcal{P}(\mathcal{C})$ is closed under retracts.

Proposition 4.4. The $\infty$-category st carries a symmetric monoidal structure characterized by the property that for $\mathcal{C}_{1}, \mathcal{C}_{2}, \mathcal{D} \in$ st, the $\infty$-category of exact functors $\operatorname{Fun}_{\text {st }}\left(\mathcal{C}_{1} \otimes \mathcal{C}_{2}, \mathcal{D}\right)$ is equivalent to the full $\infty$-subcategory of all functors $\mathcal{C}_{1} \times \mathcal{C}_{2} \rightarrow \mathcal{D}$ that preserve finite colimits in $\mathcal{C}_{1}$ and $\mathcal{C}_{2}$ separately. Furthermore, passing to the corresponding stable presentable $\infty$-categories of Ind-objects is naturally a symmetric monoidal functor.

Proof. For $\mathcal{C}_{1}, \mathcal{C}_{2} \in s t$, we define their tensor product by

$$
\mathcal{C}_{1} \otimes \mathcal{C}_{2}=\left(\operatorname{Ind}\left(\mathcal{C}_{1}\right) \otimes \operatorname{Ind}\left(\mathcal{C}_{2}\right)\right)^{c},
$$

where the tensor product of the right hand side is calculated in the $\infty$-category $\mathcal{P} r^{\mathrm{L}}$ of presentable $\infty$-categories (with morphisms the left adjoints), and the superscript ${ }^{c}$ denotes the full $\infty$-subcategory of compact objects of a presentable $\infty$-category. Since Ind $\mathcal{C}_{1} \otimes \operatorname{Ind} \mathcal{C}_{2}$ is idempotent complete and since retracts of compact objects are compact, $\left(\operatorname{Ind} \mathcal{C}_{1} \otimes \operatorname{Ind} \mathcal{C}_{2}\right)^{c}$ is idempotent complete as well. Thus the tensor product $\mathcal{C}_{1} \otimes \mathcal{C}_{2}$ is indeed an object of $s t$.

For $\mathcal{C}_{1}, \mathcal{C}_{2}, \mathcal{D} \in$ st, let $\operatorname{Fun}^{\prime}\left(\mathcal{C}_{1} \times \mathcal{C}_{2}, \mathcal{D}\right)$ be the full $\infty$-subcategory of functors $\mathcal{C}_{1} \times \mathcal{C}_{2} \rightarrow \mathcal{D}$ that preserve finite colimits in $\mathcal{C}_{1}$ and $\mathcal{C}_{2}$ separately. We claim that for $\mathcal{C}_{1}, \mathcal{C}_{2} \in$ st, the tensor product $\mathcal{C}_{1} \otimes \mathcal{C}_{2}$ corepresents the functor Fun' $\left(\mathcal{C}_{1} \times \mathcal{C}_{2},-\right)$ in the sense that for any $\mathcal{D} \in s t$, there is a canonical equivalence

$$
\operatorname{Fun}^{\prime}\left(\mathcal{C}_{1} \times \mathcal{C}_{2}, \mathcal{D}\right) \simeq \operatorname{Fun}_{s t}\left(\mathcal{C}_{1} \otimes \mathcal{C}_{2}, \mathcal{D}\right) \text {. }
$$

As a consequence, the associativity and symmetry of the tensor product $\mathcal{C}_{1} \otimes \mathcal{C}_{2}$ will immediately follow from the analogous properties of Fun'.

For $\mathcal{C}_{1}, \mathcal{C}_{2}, \mathcal{D} \in \mathcal{P} r^{\mathrm{L}}$, let $\operatorname{Fun}^{L \times L}\left(\mathcal{C}_{1} \times \mathcal{C}_{2}, \mathcal{D}\right)$ be the full $\infty$-subcategory of functors $\mathcal{C}_{1} \times \mathcal{C}_{2} \rightarrow \mathcal{D}$ that preserve colimits in $\mathcal{C}_{1}$ and $\mathcal{C}_{2}$ separately. To prove the claim, observe that the inclusion $\mathcal{D} \rightarrow \operatorname{Ind} \mathcal{D}$ induces a fully faithful functor

$$
\operatorname{Fun}^{\prime}\left(\mathcal{C}_{1} \times \mathcal{C}_{2}, \mathcal{D}\right) \rightarrow \operatorname{Fun}^{\prime}\left(\mathcal{C}_{1} \times \mathcal{C}_{2}, \operatorname{Ind} \mathcal{D}\right) \simeq \operatorname{Fun}^{L \times L}\left(\operatorname{Ind} \mathcal{C}_{1} \times \operatorname{Ind} \mathcal{C}_{2}, \operatorname{Ind} \mathcal{D}\right) .
$$

Its essential image consists of functors that preserve compact objects. By definition of the monoidal structure on the $\infty$-category $\mathcal{P} r^{\mathrm{L}}$ of presentable $\infty$-categories, we have a further equivalence

$$
\operatorname{Fun}^{L \times L}\left(\operatorname{Ind} \mathcal{C}_{1} \times \operatorname{Ind} \mathcal{C}_{2}, \operatorname{Ind} \mathcal{D}\right) \simeq \operatorname{Fun}^{\mathrm{L}}\left(\operatorname{Ind} \mathcal{C}_{1} \otimes \operatorname{Ind} \mathcal{C}_{2}, \operatorname{Ind} \mathcal{D}\right) .
$$

Since the compact objects of Ind $\mathcal{C}_{1} \otimes \operatorname{Ind} \mathcal{C}_{2}$ are generated by finite colimits of external products of compacts objects, we obtain an equivalence between $\operatorname{Fun}^{\prime}\left(\mathcal{C}_{1} \times \mathcal{C}_{2}, \mathcal{D}\right)$ and the full $\infty$-subcategory of $\operatorname{Fun}^{\mathrm{L}}\left(\operatorname{Ind} \mathcal{C}_{1} \otimes \operatorname{Ind} \mathcal{C}_{2}\right.$, Ind $\left.\mathcal{D}\right)$ consisting of functors that preserve compact objects. In other words, we have the asserted equivalence that characterizes the tensor product

$$
\operatorname{Fun}^{\prime}\left(\mathcal{C}_{1} \times \mathcal{C}_{2}, \mathcal{D}\right) \simeq \operatorname{Fun}_{s t}\left(\left(\operatorname{Ind} \mathcal{C}_{1} \otimes \operatorname{Ind} \mathcal{C}_{2}\right)^{c}, \mathcal{D}\right)
$$


Finally, the assertion that the functor Ind $: s t \rightarrow \mathcal{P} r^{\mathrm{L}}$ is symmetric monoidal is immediate from the constructions and the natural equivalence $\operatorname{Ind}\left(\mathcal{C}^{c}\right) \simeq \mathcal{C}$, for $\mathcal{C} \in \mathcal{P} r^{\mathrm{L}}$.

Remark 4.5. Given small stable idempotent complete $\infty$-categories $\mathcal{C}_{1}, \mathcal{C}_{2}$, by construction their tensor product $\mathcal{C}_{1} \otimes \mathcal{C}_{2}$ is again a small stable idempotent complete $\infty$-category. Though it is possible to consider other versions of a tensor product on small stable $\infty$-categories that need not preserve idempotent complete $\infty$ categories, our approach builds it in from the beginning.

4.2. Sheaves on fiber products. In this section, we study the $\infty$-category of sheaves on the derived fiber product of perfect stacks. The main technical result is that it is equivalent to the tensor product of the $\infty$-categories of sheaves on the factors (Theorem 4.7). The proof involves first showing that an analogous assertion holds for $\infty$-categories of perfect complexes. The remainder of the section is devoted to collecting corollaries of the main technical result.

Recall that for a perfect stack $X$, compact (equivalently, perfect or dualizable) objects in the $\infty$-category $\mathrm{QC}(X)$ form a small stable idempotent complete $\infty$ category $\mathrm{QC}(X)^{c}$, and there is canonical equivalence $\mathrm{QC}(X) \simeq \operatorname{Ind} \mathrm{QC}(X)^{c}$. Recall as well the tensor product of small stable idempotent complete $\infty$-categories, and that the functor Ind is symmetric monoidal.

Proposition 4.6. Let $X_{1}, X_{2}$ be perfect stacks. Then the external tensor product defines an equivalence

$$
\otimes: \mathrm{QC}\left(X_{1}\right)^{c} \otimes \mathrm{QC}\left(X_{2}\right)^{c} \stackrel{\sim}{\rightarrow} \mathrm{QC}\left(X_{1} \times X_{2}\right)^{c} .
$$

In other words, the $\infty$-category of perfect complexes on the product is the (small stable idempotent complete) tensor product of the $\infty$-categories of perfect complexes on the factors.

Proof. Set $X=X_{1} \times X_{2}$. By Proposition 3.24, we know that the external product takes compact objects to compact objects, and $\mathrm{QC}(X)^{c}$ is generated by external products.

Thus it suffices to verify that for $M_{i}, N_{i} \in \mathrm{QC}\left(X_{i}\right)^{c}$ we have an equivalence

$$
\operatorname{Hom}_{X}\left(M_{1} \otimes M_{2}, N_{1} \otimes N_{2}\right) \simeq \operatorname{Hom}_{X_{1}}\left(M_{1}, N_{1}\right) \otimes \operatorname{Hom}_{X_{2}}\left(M_{2}, N_{2}\right) .
$$

Using the fact that each $M_{i}$ is dualizable and $p_{2}$ satisfies the projection formula (since it is perfect), we calculate

$$
\begin{aligned}
\operatorname{Hom}_{X} & \left(p_{1}^{*} M_{1} \otimes p_{2}^{*} M_{2}, p_{1}^{*} N_{1} \otimes p_{2}^{*} N_{2}\right) \simeq \Gamma\left(X, p_{1}^{*} M_{1}^{\vee} \otimes p_{1}^{*} N_{1} \otimes p_{2}^{*} M_{2}^{\vee} \otimes p_{2}^{*} N_{2}\right) \\
& \simeq \Gamma\left(X_{2},\left(p_{2}\right)_{*}\left(p_{1}^{*} \mathcal{H} m_{X_{1}}\left(M_{1}, N_{2}\right) \otimes p_{2}^{*} \mathcal{H} m_{X_{2}}\left(M_{2}, N_{2}\right)\right)\right) \\
& \simeq \Gamma\left(X_{2}, \operatorname{Hom}_{X_{1}}\left(M_{1}, N_{1}\right) \otimes \mathcal{H o m} X_{2}\left(M_{2}, N_{2}\right)\right) \\
& \simeq \operatorname{Hom}_{X_{1}}\left(M_{1}, N_{1}\right) \otimes \operatorname{Hom}_{X_{2}}\left(M_{2}, N_{2}\right) .
\end{aligned}
$$

We now prove our main theorem which identifies $\infty$-categories of sheaves on fiber products algebraically. The proof relies on the following consequence L2, Corollary 5.5.3.4] of the $\infty$-categorical adjoint functor theorem: there is a canonical equivalence

$$
\left(\mathcal{P} r^{\mathrm{L}}\right)^{o p} \simeq \mathcal{P} r^{\mathrm{R}}
$$


between the opposite of the $\infty$-category $\mathcal{P} r^{\mathrm{L}}$ of presentable $\infty$-categories with morphisms that are left adjoints and the $\infty$-category $\mathcal{P} r^{\mathrm{R}}$ of presentable $\infty$-categories with morphisms that are right adjoints. In other words, we can reverse diagrams of presentable $\infty$-categories, in which the functors are all left adjoints, by passing to the corresponding right adjoints.

We will also use the fact that the calculation of small limits of presentable $\infty$ categories is independent of the context. Namely, by [L2, Proposition 5.5.3.13, Theorem 5.5.3.18], the forgetful functors from $\mathcal{P} r^{\mathrm{L}}, \mathcal{P} r^{\mathrm{R}}$ to all $\infty$-categories preserve small limits. In particular, given a small diagram of both left and right adjoints, the universal maps from the limit to the terms of the diagram are also both left and right adjoints.

Theorem 4.7. Let $X_{1}, X_{2}, Y$ be perfect stacks with maps $p_{1}: X_{1} \rightarrow Y, p_{2}: X_{2} \rightarrow$ $Y$. Then there is a canonical equivalence

$$
\mathrm{QC}\left(X_{1}\right) \otimes_{\mathrm{QC}(Y)} \mathrm{QC}\left(X_{2}\right) \stackrel{\sim}{\rightarrow} \mathrm{QC}\left(X_{1} \times_{Y} X_{2}\right) .
$$

Proof. To begin, consider the case $Y=\operatorname{Spec} k$, so $X_{1} \times_{Y} X_{2}=X_{1} \times X_{2}$. By Proposition 4.6 and the fact that Ind $: s t \rightarrow \mathcal{P} r^{\mathrm{L}}$ is symmetric monoidal, the external product functor provides an equivalence $\mathbb{\nabla}: \mathrm{QC}\left(X_{1}\right) \otimes \mathrm{QC}\left(X_{2}\right) \stackrel{\sim}{\rightarrow} \mathrm{QC}\left(X_{1} \times X_{2}\right)$.

To begin the case of a general perfect stack $Y$, consider the augmented cosimplicial diagram

$$
X_{1} \times_{Y} X_{2} \stackrel{\pi}{\longrightarrow} X_{1} \times X_{2} \Longrightarrow X_{1} \times Y \times X_{2} \Longrightarrow X_{1} \times Y \times Y \times X_{2} \cdots
$$

with the obvious maps constructed from the given maps $p_{1}, p_{2}$.

Applying the (contravariant) functor QC, we obtain an augmented simplicial $\infty$-category

$$
\mathrm{QC}\left(X_{1} \times_{Y} X_{2}\right)<\frac{\pi^{*}}{\mathrm{QC}}\left(X_{1} \times X_{2}\right) \longleftarrow \mathrm{QC}\left(X_{1} \times Y \times X_{2}\right) \gtreqless \ldots
$$

with structure maps given by pullbacks. By the absolute case of the theorem when $Y=\operatorname{Spec} k$, if we forget the augmentation, we obtain the simplicial $\infty$-category with simplices

$$
\mathrm{QC}\left(X_{1}\right) \otimes \mathrm{QC}(Y) \otimes \cdots \otimes \mathrm{QC}(Y) \otimes \mathrm{QC}\left(X_{2}\right)
$$

and structure maps given by tensor contractions. This is precisely the two-sided bar construction [L4, 4.5] whose geometric realization, by definition [L5, 5], calculates the tensor product of $\mathrm{QC}(Y)$-modules $\mathrm{QC}\left(X_{1}\right) \otimes_{\mathrm{QC}(Y)} \mathrm{QC}\left(X_{2}\right)$. Furthermore, the augmentation provides the natural map

$$
\mathrm{QC}\left(X_{1} \times_{Y} X_{2}\right)<\tilde{\pi}^{*} \mathrm{QC}\left(X_{1}\right) \otimes_{\mathrm{QC}(Y)} \mathrm{QC}\left(X_{2}\right),
$$

which we will prove is an equivalence.

The above geometric realization is a colimit in $\mathcal{P} r^{\mathrm{L}}$, and hence (as observed prior to the statement of the theorem) may be evaluated as a limit in the opposite category $\mathcal{P} r^{\mathrm{R}}$. Thus we find that $\mathrm{QC}\left(X_{1}\right) \otimes_{\mathrm{QC}(Y)} \mathrm{QC}\left(X_{2}\right)$ is also the totalization of the cosimplicial $\infty$-category

$$
\mathrm{QC}\left(X_{1} \times X_{2}\right) \Longrightarrow \mathrm{QC}\left(X_{1} \times Y \times X_{2}\right) \rightrightarrows \cdots
$$

with structure maps given by pushforwards. (We note for future reference that these structure maps are pushforwards along affine morphisms, hence are also colimitpreserving, i.e., left adjoints.) 
In particular, pushforward along the augmentation provides a natural functor

$$
\mathrm{QC}\left(X_{1} \times_{Y} X_{2}\right) \stackrel{\tilde{\pi}_{*}}{\longrightarrow} \mathrm{QC}\left(X_{1}\right) \otimes_{\mathrm{QC}(Y)} \mathrm{QC}\left(X_{2}\right),
$$

which is an equivalence if and only if $\tilde{\pi}^{*}$ is an equivalence.

To summarize some of the above structure, we have a diagram of commuting left (lower arrows) and right (upper arrows) adjoints

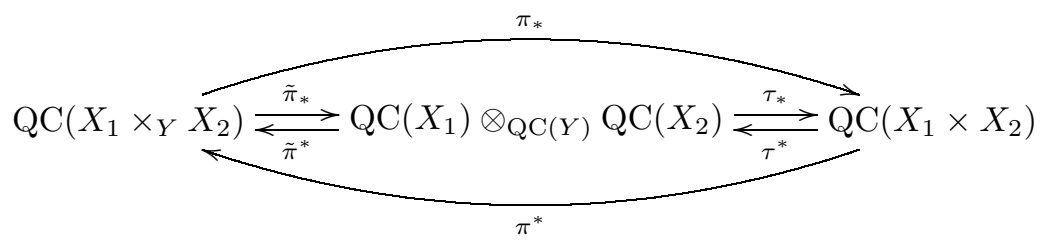

where $\tau_{*}$ is the universal map from the totalization to the zero cosimplices, and likewise, $\tau^{*}$ is the universal map from the zero simplices to the geometric realization. Thus we obtain a map of monads

$$
T_{\text {alg }}=\tau_{*} \tau^{*} \longrightarrow T_{\text {geom }}=\pi_{*} \pi^{*}
$$

acting on $\mathrm{QC}\left(X_{1} \times X_{2}\right)$.

The geometric pushforward $\pi_{*}$ is conservative and preserves colimits since $\pi$ is affine. Hence by the Barr-Beck theorem, we have a canonical equivalence

$$
\mathrm{QC}\left(X_{1} \times_{Y} X_{2}\right) \simeq \operatorname{Mod}_{T_{\text {geom }}}\left(\mathrm{QC}\left(X_{1} \times X_{2}\right)\right) .
$$

We also claim that the universal map $\tau_{*}$ is conservative and preserves colimits. For the first assertion, recall that $\tau_{*}$ is nothing more than the forgetful map from the totalization to the zero cosimplices. Since the $\infty$-categories involved are all stable, evaluating the conservatism of a functor is equivalent to determining if nonzero objects are sent to zero. But an object sent to zero in the zeroth cosimplices is sent to zero in all cosimplices and hence is equivalent to the zero-object in the limit $\infty$-category.

To see that $\tau_{*}$ preserves colimits, recall that the structure maps of our cosimplicial diagram are both right and left adjoints. It then follows (as observed prior to the statement of the theorem) that the totalization may be evaluated equivalently back in the category $\mathcal{P} r^{\mathrm{L}}$. In particular, the universal functor $\tau_{*}$ is a morphism in $\mathcal{P} r^{\mathrm{L}}$, and hence a left adjoint and so preserves colimits.

We may now apply the Barr-Beck theorem, giving a canonical equivalence

$$
\mathrm{QC}\left(X_{1}\right) \otimes_{\mathrm{QC}(Y)} \mathrm{QC}\left(X_{2}\right) \simeq \operatorname{Mod}_{T_{\text {alg }}}\left(\mathrm{QC}\left(X_{1} \times X_{2}\right)\right) .
$$

Thus it remains to show that the above morphism of monads is an equivalence. It is a straightforward diagram chase to check that the monad $T_{a l g}=\tau_{*} \tau^{*}$ is nothing more than the composition $\pi_{1}^{*} \pi_{0 *}$ of the geometric functors associated to the initial cosimplicial maps

$$
X_{1} \times X_{2} \underset{\pi_{1}}{\stackrel{\pi_{0}}{\longrightarrow}} \longrightarrow X_{1} \times Y \times X_{2} .
$$

Thus by base change, it is equivalent to the monad $T_{g e o m}=\pi_{*} \pi^{*}$. This concludes the proof of the theorem.

Corollary 4.8. For $\pi: X \rightarrow Y$ any map of perfect stacks, $\mathrm{QC}(X)$ is self-dual as a $\mathrm{QC}(Y)$-module. 
Proof. By Theorem 4.7, we have a canonical factorization

$$
\mathrm{QC}\left(X \times_{Y} X\right) \simeq \mathrm{QC}(X) \otimes_{\mathrm{QC}(Y)} \mathrm{QC}(X) .
$$

Using this identification, we can define the unit and trace by the correspondences $u=\Delta_{*} \pi^{*}: \mathrm{QC}(Y) \rightarrow \mathrm{QC}\left(X \times_{Y} X\right)$ and $\tau=\pi_{*} \Delta^{*}: \mathrm{QC}\left(X \times_{Y} X\right) \rightarrow \mathrm{QC}(Y)$, where $\Delta: X \rightarrow X \times_{Y} X$ is the relative diagonal. We need to check that the following composition is the identity:

$$
\mathrm{QC}(X) \stackrel{u \otimes \mathrm{id}}{\longrightarrow} \mathrm{QC}(X) \otimes_{\mathrm{QC}(Y)} \mathrm{QC}(X) \otimes_{\mathrm{QC}(Y)} \mathrm{QC}(X) \stackrel{\operatorname{id} \otimes \tau}{\longrightarrow} \mathrm{QC}(X) .
$$

The argument is a chase in the following diagram (with Cartesian square):

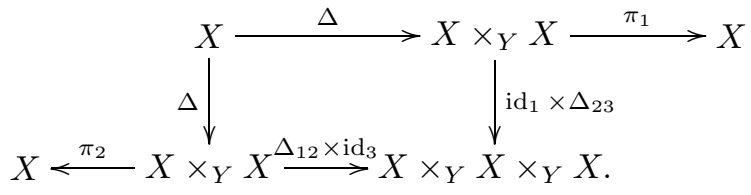

Applying base change and identities for compositions, we have the equivalences of functors

$$
\begin{aligned}
(\mathrm{id} \otimes \tau) \circ(u \otimes \mathrm{id}) & =\pi_{1 *}\left(\mathrm{id}_{1} \times \Delta_{23}\right)^{*}\left(\Delta_{12} \times \mathrm{id}_{3}\right)_{*} \pi_{2}^{*} \\
& \simeq \pi_{1 *} \Delta_{*} \Delta^{*} \pi_{2}^{*} \\
& \simeq \operatorname{id}_{\mathrm{QC}(X)} .
\end{aligned}
$$

Remark 4.9. The above argument also shows that $\mathrm{QC}(X)^{c}$ is dualizable over perfect $k$-modules $\mathrm{QC}(\operatorname{Spec} k)^{c}$ when $X$ is smooth and proper: smoothness is required for the unit in $\mathrm{QC}(X \times X)$ to be perfect, and properness is required for the trace to land in perfect $k$-modules.

For a derived stack $X$, let $\mathcal{M}, \mathcal{M}^{\prime}$ be stable presentable $\mathrm{QC}(X)$-modules. To reduce notation, we write $\operatorname{Fun}_{X}\left(\mathcal{M}, \mathcal{M}^{\prime}\right)$ for the stable presentable $\infty$-category of $\mathrm{QC}(X)$-linear colimit-preserving functors $\mathcal{M} \rightarrow \mathcal{M}^{\prime}$.

Corollary 4.10. Let $X, X^{\prime}$ and $Y$ be perfect stacks with maps $X \rightarrow Y \leftarrow X^{\prime}$. Then there is a natural equivalence of $\infty$-categories

$$
\mathrm{QC}\left(X \times_{Y} X^{\prime}\right) \stackrel{\sim}{\rightarrow} \operatorname{Fun}_{Y}\left(\mathrm{QC}(X), \mathrm{QC}\left(X^{\prime}\right)\right) .
$$

In other words, the $\infty$-category of integral kernels is equivalent to the $\infty$-category of functors.

Proof. The statement is an immediate consequence of Theorem 4.7 and the fact that $\mathrm{QC}(X)$ is self-dual (Corollary 4.8). It implies that the internal hom of $\mathrm{QC}(Y)$ modules out of $\mathrm{QC}(X)$ is calculated by tensoring with $\mathrm{QC}(X)^{\vee} \simeq \mathrm{QC}(X)$.

Remark 4.11. The equivalence of Corollary 4.10 is naturally monoidal in the following sense. For perfect stacks $X, X^{\prime}, X^{\prime \prime}$ mapping to a perfect stack $Y$, there is a convolution map

$$
\mathrm{QC}\left(X \times_{Y} X^{\prime}\right) \otimes \mathrm{QC}\left(X^{\prime} \times_{Y} X^{\prime \prime}\right) \rightarrow \mathrm{QC}\left(X \times_{Y} X^{\prime \prime}\right)
$$

given by pulling back and pushing forward with respect to the triple product $X \times_{Y}$ $X^{\prime} \times_{Y} X^{\prime \prime}$ (see Section 5.2). On the other hand, we have a composition map

$$
\operatorname{Fun}_{Y}\left(\mathrm{QC}(X), \mathrm{QC}\left(X^{\prime}\right)\right) \otimes \operatorname{Fun}_{Y}\left(\mathrm{QC}\left(X^{\prime}\right), \mathrm{QC}\left(X^{\prime \prime}\right)\right) \rightarrow \operatorname{Fun}_{Y}\left(\mathrm{QC}(X), \mathrm{QC}\left(X^{\prime \prime}\right)\right),
$$


and the equivalence of the theorem intertwines these composition maps.

Finally, for a finite simplicial set $\Sigma$, we would like to compare the formation of mapping stacks $X^{\Sigma}=\operatorname{Map}(\Sigma, X)$ (this can be viewed as the cotensoring of stacks over simplicial sets) with the formation of tensor products of $\infty$-categories $\mathcal{C} \otimes \Sigma$ (the tensoring of symmetric monoidal $\infty$-categories over simplicial sets). By the notation $\mathcal{C} \otimes \Sigma$, we mean the geometric realization of the simplicial $\infty$-category given by the constant assignment of $\mathcal{C}$ to each simplex of the simplicial set $\Sigma$.

Corollary 4.12. Let $X$ be a perfect stack and let $\Sigma$ be a finite simplicial set. Then there is a canonical equivalence

$$
\mathrm{QC}\left(X^{\Sigma}\right) \simeq \mathrm{QC}(X) \otimes \Sigma
$$

In other words, the $\infty$-category of sheaves on the mapping stack is calculated as the tensor product of the $\infty$-categories of sheaves on the simplices.

Proof. We calculate $\mathrm{QC}\left(X^{\Sigma}\right)$ by induction on the simplices as an iterated fiber product of copies of $X$ over perfect stacks (by Proposition 3.24), and apply Theorem 4.7 at each stage.

4.3. General base stacks. In this section, we extend Corollary 4.10 on integral transforms to a relative setting where the base is allowed to be an arbitrary derived stack with affine diagonal (not necessarily perfect). In order to describe integral transforms relative to such a base, we will utilize the simple behavior of $\infty$-categories of sheaves under affine base change.

Proposition 4.13. Let $Y$ be a derived stack with affine diagonal, and let $f$ : Spec $A \rightarrow Y$ be an affine map over $Y$. Then $\operatorname{Mod}_{A}$ is a self-dual QC(Y)-module. In particular, for any $X \rightarrow Y$ there is a canonical equivalence

$$
\mathrm{QC}\left(X \times_{Y} \operatorname{Spec} A\right) \simeq \mathrm{QC}(X) \otimes_{\mathrm{QC}(Y)} \operatorname{Mod}_{A} .
$$

Proof. Since $Y$ has affine diagonal, the map $f: \operatorname{Spec} A \rightarrow Y$ is a relative affine map, which implies that $f_{*}$ is colimit-preserving and conservative. Hence $f_{*}$ satisfies the monadic Barr-Beck criteria [L4, Theorem 3.4.5], implying that the natural $\operatorname{map} \operatorname{Mod}_{A} \rightarrow \operatorname{Mod}_{f_{*} f^{*}}(\mathrm{QC}(Y))$ is an equivalence. By the projection formula, the monad $f_{*} f^{*}(-)$ is equivalent to the functor $f_{*} A \otimes(-)$, with monad structure given by the algebra structure on $A$. As a consequence, we see that $\operatorname{Mod}_{A}$ is equivalent to $\operatorname{Mod}_{f_{*} A}(\mathrm{QC}(Y))$. Now Proposition 4.1 gives that $\operatorname{Mod}_{f_{*} A}(\mathrm{QC}(Y))$ is self-dual as a $\mathrm{QC}(Y)$-module.

Since the $\infty$-category $\operatorname{Mod}_{A}$ is a dualizable $\mathrm{QC}(Y)$-module it follows that the functor

$$
(-) \otimes_{\mathrm{QC}(Y)} \operatorname{Mod}_{A}
$$

commutes with limits of $\mathrm{QC}(Y)$-module categories. Thus we have the equivalences

$$
\begin{aligned}
\mathrm{QC}(X) \otimes_{\mathrm{QC}(Y)} \operatorname{Mod}_{A} & \simeq\left(\lim _{A^{\prime} \in \text { Aff } / X} \operatorname{Mod}_{A^{\prime}}\right) \otimes_{\mathrm{QC}(Y)} \operatorname{Mod}_{A} \\
& \simeq \lim _{A^{\prime} \in \text { Aff } / X}\left(\operatorname{Mod}_{A^{\prime}} \otimes_{\mathrm{QC}(Y)} \operatorname{Mod}_{A}\right) .
\end{aligned}
$$

Another application of Proposition 4.1 implies the following equivalence:

$$
\lim _{A^{\prime} \in A f f / X}\left(\operatorname{Mod}_{A^{\prime}} \otimes_{\mathrm{QC}(Y)} \operatorname{Mod}_{A}\right) \simeq \lim _{A^{\prime} \in A f f / X}\left(\operatorname{Mod}_{f_{*}^{\prime} A^{\prime} \otimes f_{*} A}(\mathrm{QC}(Y))\right) .
$$


Using that the map $f^{\prime} \times f: \operatorname{Spec} A^{\prime} \times_{Y} \operatorname{Spec} A \rightarrow Y$ is affine and that $\left(f^{\prime} \times f\right)_{*}\left(A^{\prime} \otimes\right.$ $A) \simeq f_{*}^{\prime} A^{\prime} \otimes f_{*} A$, we obtain that the above is further equivalent to

$$
\begin{gathered}
\lim _{A^{\prime} \in A f f / X}\left(\mathrm{QC}\left(\operatorname{Spec} A^{\prime} \times_{Y} \operatorname{Spec} A\right)\right) \simeq \mathrm{QC}\left(\operatorname{colim}_{A^{\prime} \in A f f} / X\left(\operatorname{Spec} A^{\prime} \times_{Y} \operatorname{Spec} A\right)\right) \\
\simeq \mathrm{QC}\left(\left(\operatorname{colim}_{A^{\prime} \in A f f} / X \operatorname{Spec} A^{\prime}\right) \times_{Y} \operatorname{Spec} A\right) \simeq \mathrm{QC}\left(X \times_{Y} \operatorname{Spec} A\right) .
\end{gathered}
$$

Here we have used that $\mathrm{QC}(-)$ sends all colimits to limits, and that $(-) \times_{Y} \operatorname{Spec} A$ commutes with colimits (since it is the left adjoint to the mapping stack over $Y$ ).

We now show that in the general setting where the base is an arbitrary derived stack with affine diagonal, functors continue to be given by integral kernels.

Theorem 4.14. Let $f: X \rightarrow Y$ be a perfect map of derived stacks with affine diagonal, and let $g: X^{\prime} \rightarrow Y$ be an arbitrary map of derived stacks. Then there is a natural map $\mathrm{QC}\left(X \times_{Y} X^{\prime}\right) \rightarrow \operatorname{Fun}_{Y}\left(\mathrm{QC}(X), \mathrm{QC}\left(X^{\prime}\right)\right)$ that is an equivalence of $\infty$-categories.

Proof. Consider the Cartesian diagram

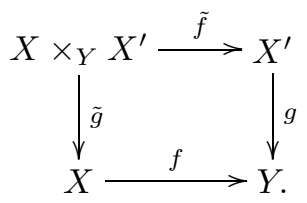

We define a functor $\mathrm{QC}\left(X \times_{Y} X^{\prime}\right) \rightarrow \mathrm{Fun}\left(\mathrm{QC}(X), \mathrm{QC}\left(X^{\prime}\right)\right)$ by sending a quasicoherent sheaf $M \in \mathrm{QC}\left(X \times_{Y} X^{\prime}\right)$ to the functor $\tilde{f}_{*}\left(M \otimes \tilde{g}^{*}-\right)$. This is a colimitpreserving functor, since $\tilde{f}$ is perfect. Furthermore, using the projection formula for the map $\tilde{f}$, this functor naturally admits the extra structure of $\mathrm{QC}(Y)$-linearity as follows:

$$
\begin{aligned}
& \tilde{f}_{*}\left(M \otimes \tilde{g}^{*}-\right) \otimes g^{*} V \simeq \tilde{f}_{*}\left(M \otimes \tilde{g}^{*}(-) \otimes \tilde{f}^{*} g^{*} V\right) \\
\simeq & \tilde{f}_{*}\left(M \otimes \tilde{g}^{*}(-) \otimes \tilde{g}^{*} f^{*} V\right) \simeq \tilde{f}_{*}\left(M \otimes \tilde{g}^{*}\left(-\otimes f^{*} V\right)\right),
\end{aligned}
$$

for any $V \in \mathrm{QC}(Y)$. Therefore, we in fact obtain a functor $\mathrm{QC}\left(X \times_{Y} X^{\prime}\right) \rightarrow$ $\operatorname{Fun}_{Y}\left(\mathrm{QC}(X), \mathrm{QC}\left(X^{\prime}\right)\right)$, and the rest of this proof will be devoted to showing it is an equivalence.

Recall that the $\infty$-category of quasi-coherent sheaves on $X^{\prime}$ is given by the limit

$$
\mathrm{QC}\left(X^{\prime}\right) \simeq \lim _{A f f / X^{\prime}} \operatorname{Mod}_{A}
$$

By working locally in the target, this provides a description of the $\infty$-category of $\mathrm{QC}(Y)$-linear functors with values in $\mathrm{QC}\left(X^{\prime}\right)$ as the limit

$$
\begin{gathered}
\operatorname{Fun}_{Y}\left(\mathrm{QC}(X), \mathrm{QC}\left(X^{\prime}\right)\right) \simeq \operatorname{Fun}_{Y}(\mathrm{QC}(X), \\
\left.\lim _{A f f / X^{\prime}} \operatorname{Mod}_{A}\right) \simeq \lim _{A f f / X^{\prime}} \operatorname{Fun}_{Y}\left(\mathrm{QC}(X), \operatorname{Mod}_{A}\right) .
\end{gathered}
$$

Likewise, we have a description of the $\infty$-category of quasi-coherent sheaves on the fiber product $X \times_{Y} X^{\prime}$ as a limit

$$
\mathrm{QC}\left(X \times_{Y} X^{\prime}\right) \simeq \lim _{A f f / X^{\prime}} \mathrm{QC}\left(X \times_{Y} \operatorname{Spec} A\right) .
$$

This follows from the fact that the functor $\mathrm{QC}(-)$ takes colimits to limits, and the fiber product functor $X \times_{Y}(-)$ commutes with all colimits (because it has a right adjoint). 
Now one can analyze the functor $\mathrm{QC}\left(X \times_{Y} X^{\prime}\right) \rightarrow \operatorname{Fun}_{Y}\left(\mathrm{QC}(X), \mathrm{QC}\left(X^{\prime}\right)\right)$ by considering the terms in the above two limits. That is, to prove the theorem, it suffices to prove it locally in the target $X^{\prime}$ : for any $\operatorname{Spec} A \rightarrow X^{\prime}$, we must show that the functor

$$
\mathrm{QC}\left(X \times_{Y} \operatorname{Spec} A\right) \rightarrow \operatorname{Fun}_{Y}\left(\mathrm{QC}(X), \operatorname{Mod}_{A}\right)
$$

is an equivalence.

We will prove this in two steps. First, we will deal with the case that the base $Y$ is affine. Afterward, we will use this case to deal with a general base $Y$.

So assume for the time being that $Y=\operatorname{Spec} B$. Then $X$ is a perfect stack over $B$, and by Corollary 4.8, $\mathrm{QC}(X)$ is a self-dual $\operatorname{Mod}_{B}$-module. Thus we have the equivalences

$$
\operatorname{Fun}_{B}\left(\mathrm{QC}(X), \operatorname{Mod}_{A}\right) \simeq \operatorname{Fun}_{B}\left(\operatorname{Mod}_{B}, \mathrm{QC}(X)^{\vee} \otimes_{B} \operatorname{Mod}_{A}\right) \simeq \mathrm{QC}(X) \otimes_{B} \operatorname{Mod}_{A} .
$$

By Proposition 4.13 we know that the functor $\mathrm{QC}(-)$ takes an affine base change to the tensor product of $\infty$-categories. Therefore we have an equivalence

$$
\mathrm{QC}\left(X \times_{B} \operatorname{Spec} A\right) \simeq \mathrm{QC}(X) \otimes_{B} \operatorname{Mod}_{A} .
$$

Putting together the above equivalences, we conclude that we have the equivalences

$$
\mathrm{QC}\left(X \times_{B} \operatorname{Spec} A\right) \simeq \mathrm{QC}(X) \otimes_{B} \operatorname{Mod}_{A} \simeq \operatorname{Fun}_{B}\left(\mathrm{QC}(X), \operatorname{Mod}_{A}\right) .
$$

This proves the theorem when $Y$ is affine.

Working locally in the base $Y$, we will now use the above discussion to prove the theorem in general. As above, since $\mathrm{QC}(-)$ and fiber products behave well with respect to colimits we can calculate the $\infty$-category of quasi-coherent sheaves on the fiber product $X \times_{Y} \operatorname{Spec} A$ as the limit

$$
\mathrm{QC}\left(X \times_{Y} \operatorname{Spec} A\right) \simeq \lim _{B \in A f f / Y} \mathrm{QC}\left(X \times_{Y} \operatorname{Spec} B \times_{Y} \operatorname{Spec} A\right) .
$$

To calculate the $\infty$-category of functors, we use the following: by Proposition 4.13, the $\infty$-category $\operatorname{Mod}_{A}$ is a dualizable $\mathrm{QC}(Y)$-module and hence the functor $\operatorname{Mod}_{A} \otimes_{\mathrm{QC}(Y)}(-)$ commutes with all limits. Therefore we have an equivalence

$$
\operatorname{Mod}_{A} \simeq \operatorname{Mod}_{A} \otimes_{\mathrm{QC}(Y)}\left(\lim _{B \in A f f / Y} \operatorname{Mod}_{B}\right) \simeq \lim _{B \in A f f / Y} \operatorname{Mod}_{A} \otimes_{\mathrm{QC}(Y)} \operatorname{Mod}_{B},
$$

and so in particular we obtain the equivalences

$$
\begin{gathered}
\operatorname{Fun}_{Y}\left(\mathrm{QC}(X), \operatorname{Mod}_{A}\right) \simeq \operatorname{Fun}_{Y}\left(\mathrm{QC}(X), \lim _{B \in A f f / Y} \operatorname{Mod}_{A} \otimes_{\mathrm{QC}(Y)} \operatorname{Mod}_{B}\right) \\
\simeq \lim _{B \in A f f / Y} \operatorname{Fun}_{Y}\left(\mathrm{QC}(X), \operatorname{Mod}_{A} \otimes_{\mathrm{QC}(Y)} \operatorname{Mod}_{B}\right) .
\end{gathered}
$$

By the adjunction between induction and restriction, and a repeated application of Proposition 4.13, we also have the equivalences

$$
\begin{aligned}
\operatorname{Fun}_{Y} & \left(\mathrm{QC}(X), \operatorname{Mod}_{A} \otimes_{\mathrm{QC}(Y)} \operatorname{Mod}_{B}\right) \\
& \simeq \operatorname{Fun}_{B}\left(\mathrm{QC}(X) \otimes_{\mathrm{QC}(Y)} \operatorname{Mod}_{B}, \operatorname{Mod}_{A} \otimes_{\mathrm{QC}(Y)} \operatorname{Mod}_{B}\right) \\
& \simeq \operatorname{Fun}_{B}\left(\mathrm{QC}\left(X \times_{Y} \operatorname{Spec} B\right), \mathrm{QC}\left(\operatorname{Spec} A \times_{Y} \operatorname{Spec} B\right)\right)
\end{aligned}
$$


Finally, by the above discussion and the affine case of the theorem with base $\operatorname{Spec} B$, we obtain the following chain of equivalences:

$$
\begin{gathered}
\mathrm{QC}\left(X \times_{Y} \operatorname{Spec} A\right) \simeq \lim _{B \in A f f / Y} \mathrm{QC}\left(X \times_{Y} \operatorname{Spec} B \times_{Y} \operatorname{Spec} A\right) \\
\simeq \lim _{B \in A f f / Y} \operatorname{Fun}_{B}\left(\mathrm{QC}\left(X \times_{Y} B\right), \mathrm{QC}\left(A \times_{Y} \operatorname{Spec} B\right)\right) \simeq \operatorname{Fun}_{Y}\left(\mathrm{QC}(X), \operatorname{Mod}_{A}\right) .
\end{gathered}
$$

Since we previously reduced the theorem to the case when $X^{\prime}=\operatorname{Spec} A$, this completes the proof.

\section{Applications}

In this section, we apply the results of Section 4 to calculate the centers and traces (and their higher $\mathcal{E}_{n}$-analogues) of symmetric monoidal $\infty$-categories of quasicoherent sheaves. We also calculate centers and traces of $\infty$-categories of linear endofunctors (equivalently by Corollary 4.10, quasi-coherent sheaves on fiber products) with monoidal structure given by composition (equivalently, convolution over the base).

5.1. Centers and traces. We begin with a discussion of the general notions of centers and traces of associative algebra objects in closed symmetric monoidal $\infty$ categories. This is a general version of the approach to topological Hochschild homology developed in [EKMM, Sh]. We then calculate the center and trace of the symmetric monoidal $\infty$-category of sheaves $\mathrm{QC}(X)$ on a perfect stack $X$ (where we think of $\mathrm{QC}(X)$ as an associative algebra object in the closed symmetric monoidal $\infty$-category $\mathcal{P} r^{\mathrm{L}}$ of presentable $\infty$-categories).

Let $\mathcal{S}$ be a symmetric monoidal presentable $\infty$-category. Recall that an associative algebra structure on $A \in \mathcal{S}$ is an object that is equivalent to the structure of the algebra over the $\mathcal{E}_{1}$-operad. We briefly recall the $\infty$-category versions of two familiar facts from classical algebra.

First, there is the notion of the opposite associative algebra $A^{\mathrm{op}} \in \mathcal{S}$. For this, recall that there is a map of operads $\tau: \mathcal{E}_{1} \rightarrow \mathcal{E}_{1}$ determined by the action of the symmetric group $\Sigma_{2}$ on the two contractible subspaces of $\mathcal{E}_{1}(2)$, and such that $\tau \circ \tau$ is equivalent to the identity. Given an $\mathcal{E}_{1}$-algebra $A$, the pullback $\tau^{*} A$ is by definition the opposite $\mathcal{E}_{1}$-algebra $A^{\text {op }}$.

Second, given two associative algebras $A, B \in \mathcal{S}$, their monoidal product $A \otimes B \in$ $\mathcal{S}$ carries a natural associative algebra structure. For this, recall that given two algebras $A, B$ over any topological operad $\mathcal{O}$, we have the structure of an $\mathcal{O} \times \mathcal{O}$ algebra on the monoidal product $A \otimes B$. Since the term-wise diagonal map gives a map of operads $\mathcal{O} \rightarrow \mathcal{O} \times \mathcal{O}$, we obtain an $\mathcal{O}$-algebra structure on $A \otimes B$ by restriction along the diagonal.

Furthermore, any associative algebra $A \in \mathcal{S}$ is a left (as well as a right) module object over the associative algebra $A \otimes A^{\text {op }}$ via left and right multiplication.

Now assume further that $\mathcal{S}$ is a closed symmetric monoidal $\infty$-category. Then we have internal hom objects [L4, 2.7], and given $A \otimes A^{\mathrm{op}}$-modules $M, N$, we can define the $A \otimes A^{\mathrm{op}}$-linear morphism object $\mathcal{H o m}_{A \otimes A^{\mathrm{op}}}(M, N) \in \mathcal{S}$. Likewise, given left and right $A \otimes A^{\mathrm{op}}$-modules $M, N \in \mathcal{S}$ we have a pairing $M \otimes_{A \otimes A^{\text {op }}} N \in \mathcal{S}$ defined by the two-sided bar construction [L4, 4.5] over $A \otimes A^{\text {op }}$.

Definition 5.1. Let $A$ be an associative algebra object in a closed symmetric monoidal $\infty$-category $\mathcal{S}$. 
(1) The derived center or Hochschild cohomology $\mathcal{Z}(A)=\mathrm{HH}^{*}(A) \in \mathcal{S}$ is the endomorphism object $\mathcal{E} n d_{A \otimes A^{\text {op }}}(A)$ of $A$ as an $A$-bimodule.

(2) The derived trace or Hochschild homology $\mathcal{T r}(A)=\mathrm{HH}_{*}(A) \in \mathcal{S}$ is the pairing object $A \otimes_{A \otimes A^{\text {op }}} A$ of $A$ with itself as an $A$-bimodule.

In general, the center $\mathcal{Z}(A)$ is again an associative algebra object in $\mathcal{S}$, and the trace $\operatorname{Tr}(A)$ is an $A$-module object in $\mathcal{S}$. Furthermore, $\mathcal{Z}(A)$ comes with a canonical central morphism

$$
\mathfrak{z}: \mathcal{Z}(A) \longrightarrow A \quad F \longmapsto F\left(1_{A}\right)
$$

while $\operatorname{Tr}(A)$ comes with a canonical trace morphism

$$
\mathfrak{t r}: A \longrightarrow \mathcal{T r}(A) \quad A \longmapsto A \otimes_{A \otimes A} 1_{A}
$$

coequalizing left and right multiplication. When $A$ is in fact symmetric, $\mathcal{Z}(A)$ and $\mathcal{T} r(A)$ are again naturally symmetric algebra objects in $\mathcal{S}$, though the symmetric algebra structure on $\mathcal{Z}(A)$ is different from its general associative algebra structure.

5.1.1. Cyclic bar construction. It is very useful to have versions of the Hochschild chain and cochain complexes which calculate the trace and center. In the setting of an associative algebra object $A$ in a monoidal $\infty$-category $\mathcal{S}$, they will take the form of a simplicial object $\mathbf{N}_{*}^{c y c}(A)$ and cosimplicial object $\mathbf{N}_{c y c}^{*}(A)$ such that the geometric realization colim $\mathbf{N}_{*}^{c y c}(A)$ is the trace $\mathcal{T} r(A)$ and the totalization $\lim \mathbf{N}_{c y c}^{*}(A)$ is the center $\mathcal{Z}(A)$. (Note that simplicial and cosimplicial objects here are taken in the $\infty$-categorical sense, and so the diagram identities hold up to coherent homotopies as in, e.g., $\mathrm{A}$, where the cyclic bar construction for $A_{\infty}$ $H$-spaces is developed.)

We construct the simplicial object $\mathbf{N}_{*}^{c y c}(A)$ and cosimplicial object $\mathbf{N}_{c y c}^{*}(A)$ as follows. Consider the adjunction

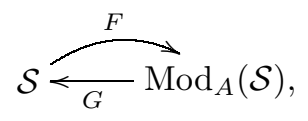

where $F(-)=A \otimes-$ is the induction, and $G$ is the forgetful functor. It determines a comonad $S \simeq F G$ acting on $\operatorname{Mod}_{A}(\mathcal{S})$.

As in classical algebra, for any $A$-module $M$, the comonad $S$ provides a canonical augmented simplicial object $C_{*}(M)$ with terms

$$
C_{n-1}(M) \simeq(F G)^{n}(M) \simeq A^{\otimes n} \otimes M
$$

such that the geometric realization of $C_{*}(M)$ is naturally equivalent to $M$. We will say that $C_{*}(M)$ is a simplicial resolution of $M$.

We can apply this technique to produce the familiar cyclic bar construction. Consider the special case of the above adjunction

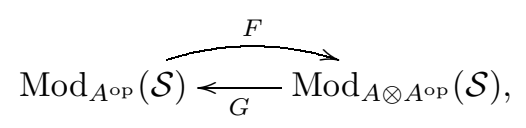

where $F(-)=A \otimes-$ again is the induction, and $G$ is the forgetful functor from $A$ bimodules to right $A$-modules. Using the comonad $S \simeq F G$, we obtain a simplicial resolution $C_{*}(A)$ of the $A$-bimodule $A$ whose terms $C_{n-1}(A) \simeq A^{\otimes n+1}$ are $A$ bimodules which are free as left $A$-modules. 
Now recall that the trace $\mathcal{T} r(A)$ is defined by the self-pairing $A \otimes_{A \otimes A^{\circ p}} A$. Since the tensor product commutes with colimits, in particular geometric realizations, we calculate

$$
\mathcal{T} r(A)=A \otimes_{A \otimes A^{\text {op }}} A \simeq A \otimes_{A \otimes A^{\text {op }}}\left|C_{*}(A)\right| \simeq\left|A \otimes_{A \otimes A^{\text {op }}} C_{*}(A)\right| .
$$

Thus the geometric realization of $A \otimes A \otimes A^{\text {op }} C_{*}(A)$ calculates the trace $\operatorname{Tr}(A)$.

We write $\mathbf{N}_{*}^{c y c}(A)$ for the simplicial object $A \otimes_{A \otimes A^{\text {op }}} C_{*}(A)$ and refer to it as the Hochschild chain complex. Since $A$ is free as a right $A$-module, the terms of the simplicial object $C_{*}(A)$ are free as $A$-bimodules. Thus we can evaluate the terms of the Hochschild chain complex

$$
\mathbf{N}_{n}^{c y c}(A)=A \otimes_{A \otimes A^{\text {op }}} C_{n}(A) \simeq A \otimes_{A \otimes A^{\text {op }}} A^{\otimes n+2} \simeq A^{\otimes n+1} .
$$

In particular, there are equivalences $\mathbf{N}_{0}^{c y c}(A) \simeq A$ and $\mathbf{N}_{1}^{c y c}(A) \simeq A \otimes A$, and the two simplicial maps $A \otimes A \rightarrow A$ are the multiplication and the opposite multiplication of $A$.

Similarly, recall that the center $\mathcal{Z}(A)$ is defined by the endomorphisms $\mathcal{E} n d_{A \otimes A^{\text {op }}}(A)$. Since morphisms take colimits in the domain to limits, in particular geometric realizations to totalizations, we calculate

$$
\mathcal{Z}(A)=\mathcal{E}_{n d_{A \otimes A^{\text {op }}}}(A) \simeq \mathcal{H}_{o m_{A \otimes A^{o p}}}\left(\left|C_{*}(A)\right|, A\right) \simeq\left|\mathcal{H} o m_{A \otimes A^{o p}}\left(C_{*}(A), A\right)\right| .
$$

Thus the totalization of $\mathcal{H o m}_{A \otimes A^{o p}}\left(C_{*}(A), A\right)$ calculates the center $\mathcal{Z}(A)$.

We write $\mathbf{N}_{c y c}^{*}(A)$ for the cosimplicial object $\mathcal{H o m}_{A \otimes A^{o p}}\left(C_{*}(A), A\right)$ and refer to it as the Hochschild cochain complex. As before, we can evaluate the terms of the Hochschild cochain complex:

$$
\mathbf{N}_{c y c}^{n}(A)=\mathcal{H o m}_{A \otimes A^{o p}}\left(C_{n}(A), A\right) \simeq \mathcal{H o m}_{A \otimes A^{o p}}\left(A^{\otimes n+2}, A\right) \simeq \mathcal{H} o m\left(A^{\otimes n}, A\right) .
$$

In particular, there are equivalences $\mathbf{N}_{c y c}^{0}(A) \simeq A$ and $\mathbf{N}_{c y c}^{1}(A) \simeq \mathcal{H o m}(A, A)$, and the two cosimplicial maps $A \rightarrow \mathcal{H o m}(A, A)$ are induced by the left and right multiplication of $A$.

5.1.2. Centers and traces for monoidal $\infty$-categories. We will apply the above constructions in the following setting. We will always take $\mathcal{S}$ to be the $\infty$-category $\mathcal{P} r^{\mathrm{L}}$ of presentable $\infty$-categories (with morphisms being left adjoints). Then a monoidal presentable $\infty$-category $\mathcal{C}$ is an associative algebra object in $\mathcal{P} r^{\mathrm{L}}$. Thus we have the notion of its center (or Hochschild cohomology category) $\mathcal{Z}(\mathcal{C})=\operatorname{Fun}_{\mathcal{C} \otimes \mathcal{C}^{\text {op }}}(\mathcal{C}, \mathcal{C}) \in$ $\mathcal{P} r^{\mathrm{L}}$, and its trace (or Hochschild homology category) $\mathcal{T} r(\mathcal{C})=\mathcal{C} \otimes \mathcal{C} \otimes \mathcal{C}^{\circ} \mathcal{C} \in \mathcal{P} r^{\mathrm{L}}$.

Now we will specialize further to a geometric setting. Let $X$ be a perfect stack, and take $\mathcal{C}$ to be the presentable stable $\infty$-category $\mathrm{QC}(X)$ equipped with its (symmetric) monoidal tensor product. To calculate the center and trace of $\mathrm{QC}(X)$, we introduce the loop space

$$
\mathcal{L} X=\operatorname{Map}\left(S^{1}, X\right) \simeq X \times_{X \times X} X,
$$

where the fiber product is along two copies of the diagonal map.

Corollary 5.2. Let $X$ be a perfect stack, and equip $\mathrm{QC}(X)$ with its (symmetric) monoidal algebra structure given by the tensor product. Then there are canonical equivalences (of symmetric monoidal) $\infty$-categories:

$$
\mathrm{QC}(\mathcal{L} X) \simeq \mathcal{Z}(\mathrm{QC}(X)) \simeq \mathcal{T} r(\mathrm{QC}(X)) .
$$


Proof. By Theorem 4.7 we know that $\operatorname{Tr}(\mathrm{QC}(X))$, which is a tensor product, is also calculated by a fiber product

$$
\mathcal{T r}(\mathrm{QC}(X)) \simeq \mathrm{QC}\left(X \times_{X \times X} X\right) .
$$

On the other hand, by Corollary 4.10, we know that $\mathcal{Z}(\mathrm{QC}(X))$, which consists of functors, is also calculated by a tensor product

$$
\mathcal{Z}(\mathrm{QC}(X)) \simeq \mathcal{T} r(\mathrm{QC}(X)) .
$$

5.2. Centers of convolution categories. Let $p: X \rightarrow Y$ be a map of perfect stacks satisfying descent. Consider the convolution diagram

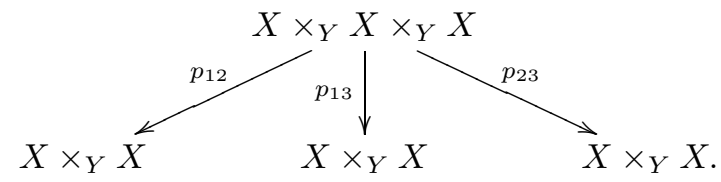

Equip $\mathrm{QC}\left(X \times_{Y} X\right)$ with the monoidal product defined by convolution

$$
M \star N=p_{13 *}\left(p_{12}^{*}(M) \otimes p_{23}^{*}(N)\right) .
$$

By Theorem 4.14, we have a monoidal equivalence

$$
\mathrm{QC}\left(X \times_{Y} X\right) \simeq \operatorname{Fun}_{Y}(\mathrm{QC}(X), \mathrm{QC}(X)) .
$$

Consider the fundamental correspondence

$$
\mathcal{L} Y=Y \times_{Y \times Y} Y \stackrel{\pi}{\longleftarrow} \mathcal{L} Y \times_{Y} X=X \times_{X \times Y} X \stackrel{\delta}{\longrightarrow} X \times_{Y} X,
$$

where the maps are defined by the formulas

$$
\pi=\operatorname{id}_{\mathcal{L} Y} \times_{\operatorname{id}_{Y}} p=p \times_{p \times \operatorname{id}_{Y}} p, \quad \delta=\operatorname{id}_{X} \times_{\pi_{Y}} \operatorname{id}_{X},
$$

where $\pi_{Y}: X \times Y \rightarrow Y$ is the obvious projection.

Passing to sheaves, we obtain a diagram

$$
\mathrm{QC}(\mathcal{L} Y) \stackrel{\pi^{*}}{\longrightarrow} \mathrm{QC}\left(\mathcal{L} Y \times_{Y} X\right) \stackrel{\delta_{*}}{\longrightarrow} \mathrm{QC}\left(X \times_{Y} X\right),
$$

which by Theorem 4.14 admits the interpretation

$$
\begin{aligned}
\operatorname{Fun}_{Y \times Y}(\mathrm{QC}(Y), \mathrm{QC}(Y)) \stackrel{\pi^{*}}{\longrightarrow} \operatorname{Fun}_{X \times Y}(\mathrm{QC}(X), \mathrm{QC}(X)) \\
\stackrel{\delta_{*}}{\longrightarrow} \operatorname{Fun}_{Y}(\mathrm{QC}(X), \mathrm{QC}(X)) .
\end{aligned}
$$

where $\pi^{*}$ is the $\mathrm{QC}(X)$-linear induction, and $\delta_{*}$ forgets the $\mathrm{QC}(X)$-linear structure.

The aim of this section is to prove the following.

Theorem 5.3. Suppose $p: X \rightarrow Y$ is a map of perfect stacks satisfying descent. Then there is a canonical equivalence

$$
\mathcal{Z}\left(\mathrm{QC}\left(X \times_{Y} X\right)\right) \simeq \mathrm{QC}(\mathcal{L} Y)
$$

such that the forgetful functor $\mathfrak{z}: \mathcal{Z}\left(\mathrm{QC}\left(X \times_{Y} X\right)\right) \rightarrow \mathrm{QC}\left(X \times_{Y} X\right)$ is given by the correspondence $\delta_{*} \pi^{*}: \mathrm{QC}(\mathcal{L} Y) \rightarrow \mathrm{QC}\left(X \times_{Y} X\right)$. 
The proof occupies the remainder of this section. We break up the argument into a general discussion and then its specific application.

At the end of the section, we also explain an analogous description of traces, conditional on a still to be developed version of Grothendieck duality in the derived setting. Namely, assuming further that $p: X \rightarrow Y$ is proper with invertible dualizing sheaf and that Grothendieck duality holds, we explain how to deduce an expected canonical equivalence

$$
\mathcal{T} r\left(\mathrm{QC}\left(X \times_{Y} X\right)\right) \simeq \mathrm{QC}(\mathcal{L} Y)
$$

such that the trace $\mathfrak{t r}: \mathrm{QC}\left(X \times_{Y} X\right) \rightarrow \mathcal{T} r\left(\mathrm{QC}\left(X \times_{Y} X\right)\right)$ is given by the correspondence $\pi_{*} \delta^{*}: \mathrm{QC}\left(X \times_{Y} X\right) \rightarrow \mathrm{QC}(\mathcal{L} Y)$.

5.2.1. Relative cyclic bar construction. For the proof of Theorem 5.3, it will be useful to introduce relative versions of the Hochschild chain and cochain complexes. In general, the setup for the construction will be a map of associative algebra objects $B \rightarrow A$ in a monoidal $\infty$-category $\mathcal{S}$. The usual Hochschild chain and cochain complexes introduced in the previous section will correspond to the case when $B$ is the unit $1_{\mathcal{S}}$.

Consider the adjunction

$$
\operatorname{Mod}_{B \otimes A^{\text {op }}} \underset{(\mathcal{S}) \leftarrow \operatorname{Mod}_{A \otimes A^{\text {op }}}(\mathcal{S}),}{\vec{F}}
$$

where $F(-)=A \otimes_{B}-$ is the induction and $G$ is the forgetful functor from $A$ bimodules to $B \otimes A^{\mathrm{op}}$-modules. Using the comonad $S \simeq F G$, we obtain a simplicial resolution $C_{*}^{B}(A)$ of the $A$-bimodule $A$ with terms

$$
C_{n-1}^{B}(A) \simeq A \otimes_{B} \otimes \cdots \otimes_{B} A, \quad \text { with } n+1 \text { terms. }
$$

To reduce the notation, we will denote the above expression by $A_{B}^{\otimes n+1}$.

As before, the geometric realization of $A \otimes A \otimes A^{\text {op }} C_{*}^{B}(A)$ calculates the trace $\mathcal{T r}(A)$, and the totalization of $\mathcal{H o m}_{A \otimes A^{o p}}\left(C_{*}^{B}(A), A\right)$ calculates the center $\mathcal{Z}(A)$. We write $\mathbf{N}_{*}^{B}(A)$ for the simplicial object $A \otimes A \otimes A^{\text {op }} C_{*}^{B}(A)$ and refer to it as the relative Hochschild chain complex. Similarly, we write $\mathbf{N}_{B}^{*}(A)$ for the cosimplicial object $\mathcal{H o m}_{A \otimes A^{o p}}\left(C_{*}^{B}(A), A\right)$ and refer to it as the relative Hochschild cochain complex.

Continuing as before, we can evaluate the terms of the relative Hochschild chain complex

$$
\mathbf{N}_{n}^{B}(A)=A \otimes_{A \otimes A^{\text {op }}} C_{n}^{B}(A) \simeq A \otimes_{A \otimes A^{\text {op }}} A_{B}^{\otimes n+2} \simeq B \otimes_{B \otimes B^{\text {op }}} A_{B}^{\otimes n+1} .
$$

Similarly, we can evaluate the terms of the relative Hochschild cochain complex

$$
\begin{aligned}
\mathbf{N}_{B}^{n}(A)=\mathcal{H o m}_{A \otimes A^{o p}}\left(C_{n}^{B}(A), A\right) & \simeq \mathcal{H o m}_{A \otimes A^{o p}}\left(A_{B}^{\otimes n+2}, A\right) \\
& \simeq \mathcal{H o m} m_{B \otimes B^{\text {op }}}\left(A_{B}^{\otimes n+1}, B\right) .
\end{aligned}
$$

5.2.2. Proof of Theorem 5.3. Now we will apply the preceding formalism to calculate the center of the monoidal $\infty$-category $\mathrm{QC}\left(X \times_{Y} X\right)$. Recall that our aim is to show that there is a canonical equivalence

$$
\mathcal{Z}\left(\mathrm{QC}\left(X \times_{Y} X\right)\right) \simeq \mathrm{QC}(\mathcal{L} Y)
$$

where $\mathcal{L} Y$ is the loop space of $Y$. 
Consider the symmetric monoidal $\infty$-category $\mathrm{QC}(X)$ together with the monoidal functor

$$
\Delta_{*}: \mathrm{QC}(X) \rightarrow \mathrm{QC}\left(X \times_{Y} X\right)
$$

obtained via pushforward along the relative diagonal $\Delta: X \rightarrow X \times_{Y} X$.

Set $A=\mathrm{QC}\left(X \times_{Y} X\right)$ and $B=\mathrm{QC}(X)$. By the preceding discussion, the center $\mathcal{Z}(A)$ is the limit of the relative Hochschild cochain complex $\mathbf{N}_{B}^{*}(A)$. Furthermore, its terms can be calculated:

$$
\mathbf{N}_{B}^{n}(A) \simeq \mathcal{H}_{\text {om }} \otimes B^{\mathrm{op}}\left(A_{B}^{\otimes n+1}, B\right)
$$

Applying the results of Section 4, we can rewrite each term in the form

$$
\mathbf{N}_{B}^{n}(A) \simeq \mathrm{QC}\left(\mathcal{L} Y \times_{Y} X \times_{Y} \cdots \times_{Y} X\right), \quad \text { with } n+1 \text { copies of } X .
$$

Here we have used the elementary identification

$$
\mathcal{L} Y \times_{Y} X \times_{Y} \cdots \times_{Y} X \simeq X \times_{X \times X}\left(\left(X \times_{Y} X\right) \times_{X} \cdots \times_{X}\left(X \times_{Y} X\right)\right),
$$

where the left hand side has $n+1$ copies of $X$, and the right hand side has $n+1$ copies of $X \times_{Y} X$.

We conclude that the terms of $\mathbf{N}_{B}^{*}(A)$ are nothing more than the terms of the cosimplicial $\infty$-category obtained by applying $\mathrm{QC}(-)$ to the Cech simplicial stack induced by the map

$$
\tilde{p}: \mathcal{L} Y \times_{Y} X \rightarrow \mathcal{L} Y
$$

obtained by base change from the original map $p: X \rightarrow Y$. It is straightforward to check that under this identification the coboundary maps are given by the usual

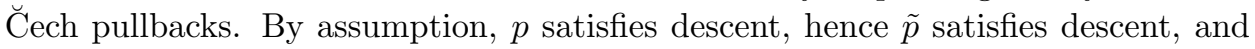
thus the totalization $\lim \mathbf{N}_{B}^{*}(A)$ also calculates the $\infty$-category $\mathrm{QC}(\mathcal{L} Y)$.

Finally, note that under this identification, the composition $\delta_{*} \pi^{*}: \mathrm{QC}(\mathcal{L} Y) \rightarrow$ $\mathrm{QC}\left(X \times_{Y} X\right)$ corresponds to the composition

$$
\mathrm{QC}(\mathcal{L} Y) \simeq \lim \mathbf{N}_{B}^{*}(A) \rightarrow \mathbf{N}_{B}^{0}(A) \rightarrow \mathbf{N}_{c y c}^{0}(A) \simeq \mathrm{QC}\left(X \times_{Y} X\right),
$$

which is precisely the central functor $\mathfrak{z}$. This concludes the proof of Theorem 5.3

5.2.3. Traces and Grothendieck duality. Finally, we explain here an analogous description of traces, conditional on a still to be developed version of Grothendieck duality in the derived setting. Namely, assuming further that $p: X \rightarrow Y$ is proper with invertible dualizing sheaf and that Grothendieck duality holds, we explain how to deduce an expected canonical equivalence

$$
\mathcal{T} r\left(\mathrm{QC}\left(X \times_{Y} X\right)\right) \simeq \mathrm{QC}(\mathcal{L} Y)
$$

such that the trace $\mathfrak{t r}: \mathrm{QC}\left(X \times_{Y} X\right) \rightarrow \mathcal{T} r\left(\mathrm{QC}\left(X \times_{Y} X\right)\right)$ is given by the correspondence $\pi_{*} \delta^{*}: \mathrm{QC}\left(X \times_{Y} X\right) \rightarrow \mathrm{QC}(\mathcal{L} Y)$.

We continue with the notation from the proof of Theorem 5.3 and the preceding sections. We will show that the geometric realization colim $\mathbf{N}_{*}^{B}(A)$ of the relative Hochschild chain complex also calculates the $\infty$-category $\mathrm{QC}(\mathcal{L} Y)$. As before, applying the results of Section 4, we can rewrite the terms of $\mathbf{N}_{*}^{B}(A)$ in the form

$$
\mathbf{N}_{n}^{B}(A) \simeq \mathrm{QC}\left(\mathcal{L} Y \times_{Y} X \times_{Y} \cdots \times_{Y} X\right) \quad \text { with } n+1 \text { copies of } X .
$$

Furthermore, it is straightforward to check that under this identification the boundary maps of $\mathbf{N}_{*}^{B}(A)$ are given by the pushforwards $\mathfrak{p}_{n *}$, which are right adjoints to the usual C̆ech pullbacks $\mathfrak{p}_{n}^{*}$.

To reduce notation, set $X_{n}=X \times_{Y} \cdots \times_{Y} X$ with $n+1$ copies of $X$. 
Now suppose that $p: X \rightarrow Y$ is proper and has an invertible dualizing complex (Gorenstein). Then we expect Grothendieck duality to hold in the following form: the pushforwards $\mathfrak{p}_{n *}$ are also left adjoints to the pullbacks

$$
\mathfrak{p}_{n}^{!}(-) \simeq \mathfrak{p}_{n}^{*}\left(-\otimes \omega_{\mathcal{L} Y \times_{Y} X_{n-1} / Y}^{-1}\right) \otimes \omega_{\mathcal{L} Y \times_{Y} X_{n} / Y},
$$

where $\omega_{\mathcal{L} Y \times_{Y} X_{n} / Y}$ denotes the relative dualizing sheaf of $\mathcal{L} Y \times_{Y} X_{n} \rightarrow Y$. Under this assumption, we find that the limit $\mathrm{QC}(\mathcal{L} Y)$ of the cosimplicial $\infty$-category $\left(\mathrm{QC}\left(\mathcal{L} Y \times_{Y} X_{n}\right), \mathfrak{p}_{n}^{*}\right)$ admits the following alternative description.

First, we can identify the cosimplicial $\infty$-category $\left(\mathrm{QC}\left(\mathcal{L} Y \times_{Y} X_{n}\right), \mathfrak{p}_{n}^{*}\right)$ with the cosimplicial $\infty$-category $\left(\mathrm{QC}\left(\mathcal{L} Y \times_{Y} X_{n}, \mathfrak{p}_{n}^{!}\right)\right.$via tensoring by the inverse of the relative dualizing sheaf $\omega_{\mathcal{L} Y \times_{Y} X_{n} / Y}$ on each simplex. In particular, we obtain an identification of their limits.

Second, we can consider the cosimplicial $\infty$-category $\left(\mathrm{QC}\left(\mathcal{L} Y \times_{Y} X_{n}\right), \mathfrak{p}_{n}^{!}\right)$as a diagram in the $\infty$-category $\mathcal{P} r^{\mathrm{R}}$ of presentable $\infty$-categories (with morphisms being right adjoints). By [L2, Theorem 5.5.3.18], the calculation of the limit of the diagram does not depend on this choice of context. Then we can pass to the opposite $\infty$-category $\mathcal{P} r^{\mathrm{L}}$ of presentable $\infty$-categories (with morphisms being left adjoints). To calculate a limit in $\mathcal{P} r^{\mathrm{R}}$ is the same as to calculate a colimit in $\mathcal{P} r^{\mathrm{L}}$. But we have seen that the trace $\operatorname{Tr}(\mathcal{H})$ is precisely the colimit of the dual simplicial diagram $\left(\mathrm{QC}\left(\mathcal{L} Y \times_{Y} X_{n}\right), \mathfrak{p}_{n *}\right)$. Thus we conclude that the geometric realization colim $\mathbf{N}_{*}^{B}(A)$ also calculates the $\infty$-category $\mathrm{QC}(\mathcal{L} Y)$.

Finally, note that under this identification, the composition $\pi_{*} \delta^{*}: \mathrm{QC}\left(X \times_{Y}\right.$ $X) \rightarrow \mathrm{QC}(\mathcal{L} Y)$ corresponds to the composition

$$
\mathrm{QC}\left(X \times_{Y} X\right) \simeq \mathbf{N}_{0}^{c y c}(A) \rightarrow \mathbf{N}_{0}^{B}(A) \rightarrow \operatorname{colim} \mathbf{N}_{*}^{c y c}(A) \simeq \mathrm{QC}(\mathcal{L} Y),
$$

which is precisely the trace $\mathfrak{t r}$.

5.3. Higher centers. Unlike in classical algebra, for an algebra object in an $\infty$ category, commutativity is an additional structure rather than a property. More precisely, the forgetful functor from $\mathcal{E}_{\infty}$-algebras to $\mathcal{E}_{1}$-algebras is conservative but not fully faithful. Given an $\mathcal{E}_{\infty}$-algebra $A$ in a symmetric monoidal $\infty$-category $\mathcal{S}$, the center $\mathcal{Z}(A)$ that we have studied to this point does not involve the commutativity of $A$. More precisely, it only depends upon the $\mathcal{E}_{1}$-algebra underlying A.

In this section, we discuss higher versions of the center where we forget less commutativity. For a perfect stack $X$, we calculate the $\mathcal{E}_{n}$-center of the $\mathcal{E}_{n}$-category underlying the $\mathcal{E}_{\infty}$-category $\mathrm{QC}(X)$. A detailed treatment of the foundations of the subject may be found in Chapter 2 of [F1. Here we summarize only what is required for our consideration of the $\mathcal{E}_{n}$-center of $\mathrm{QC}(X)$.

Remark 5.4. As noted in the introduction, after the completion of this manuscript, the paper [L5] was revised to include a thorough treatment of $\infty$-categorical operads and their algebras, and the paper [L7] treats in great detail the specific case of the $\mathcal{E}_{n}$-operads. We refer the reader to these preprints for further details.

Let $F$ be a topological operad. We define a topological category $\mathcal{F}$ with objects finite pointed sets and morphism spaces given by

$$
\operatorname{Map}_{\mathcal{F}}\left(J_{*}, I_{*}\right)=\coprod_{f: J_{*} \rightarrow I_{*}} \prod_{I} F\left(f^{-1}\{i\}\right) .
$$


We then obtain an $\infty$-category, also denoted by $\mathcal{F}$, by applying the singular functor to the mapping spaces to get a simplicial category and then taking the simplicial nerve.

Definition 5.5 (F1]). An $\mathcal{F}$-monoidal structure on an $\infty$-category $\mathcal{C}$ consists of a functor $p: \mathcal{F} \rightarrow$ Cat $_{\infty}$ with an identification $\mathcal{C} \simeq p\left(1_{*}\right)$ such that the natural map $p\left(J_{*}\right) \rightarrow \prod_{J} p\left(1_{*}\right)$ resulting from a choice of a point $x \in F(J)$ is an equivalence.

We will refer to the $\infty$-category $\mathcal{C}$ equipped with an $\mathcal{F}$-monoidal structure as an $\mathcal{F}$-category. This construction will be of particular interest to us when $\mathcal{F}$ is the little $n$-disks operad $\mathcal{E}_{n}$.

To discuss Hochschild cohomology, we must next introduce the notion of operadic modules.

To this end, let $\operatorname{Fin}_{*}$ be the category of finite based sets. Let Fin ${ }_{+}$be the category of "doubly-based sets" with objects finite based sets $I_{*}$ and $(I \amalg+)_{*}$, and morphisms $\alpha$ of the underlying based sets such that $+\in \alpha^{-1}(+)$, and either $\alpha(+)=+$ or $\alpha(+)=*$.

Given a topological operad $F$, recall the $\infty$-category $\mathcal{F}$ introduced above. We also define the $\infty$-category $\mathcal{F}_{+}$to be the fiber product of $\infty$-categories

$$
\mathcal{F}_{+}=\mathcal{F} \times_{\mathrm{Fin}_{*}} \operatorname{Fin}_{+} \text {. }
$$

Definition 5.6. Let $\mathcal{C}$ be an $\mathcal{F}$-category. An $\mathcal{F}-\mathcal{C}$-module structure on an $\infty$ category $\mathcal{M}$ is a functor $q: \mathcal{F}_{+} \rightarrow$ Cat $_{\infty}$ extending the $\mathcal{F}$-monoidal structure on $\mathcal{C}$, together with an identification $q(+) \simeq \mathcal{M}$ such that the natural map $q\left((I \amalg+)_{*}\right) \rightarrow$ $\mathcal{C}^{I} \times \mathcal{M}$ is an equivalence for any $I$.

Example 5.7. When $\mathcal{F}$ is the $\mathcal{E}_{1}$-operad, the notion of an $\mathcal{F}-\mathcal{C}$-module coincides with that of a $\mathcal{C}$-bimodule: it is an $\infty$-category left and right tensored over $\mathcal{C}$. When $\mathcal{F}$ is the $\mathcal{E}_{\infty}$-operad, the notion coincides with that of a left (or equivalently right) module.

There is a similar definition of an $\mathcal{F}$-algebra and an $\mathcal{F}$ - $A$-module in a symmetric monoidal or $\mathcal{F}$-monoidal $\infty$-category $\mathcal{M}$ generalizing the definition given above. This requires the notion of an $\mathcal{F}$-lax monoidal functor (see F1, Chapter 2, Definition 3.10]). The simplification of the previous definition is available because $\mathrm{Cat}_{\infty}$ is equipped with the Cartesian monoidal structure. We will avail ourselves of this greater generality in the following definition, although the only case that will concern us in the following is when $\mathcal{M}$ is either $\mathrm{Cat}_{\infty}$ or $\mathcal{P} r^{\mathrm{L}}$.

Now let $\mathcal{M}$ be a presentable symmetric monoidal $\infty$-category whose monoidal structure distributes over colimits. Let $A$ be an $\mathcal{F}$-algebra in $\mathcal{M}$, and let $\operatorname{Mod}_{A}^{\mathcal{F}}(\mathcal{M})$ be the $\infty$-category of $\mathcal{F}$-A-modules in $\mathcal{M}$ (see [F1, Chapter 2, Definition 4.3]). Under the above assumptions, the $\infty$-category $\operatorname{Mod}_{A}^{\mathcal{F}}(\mathcal{M})$ is naturally tensored over $\mathcal{M}$.

Definition 5.8. For an $\mathcal{F}$-algebra $A$ in $\mathcal{M}$, we define the $\mathcal{F}$-Hochschild cohomology

$$
\operatorname{HH}_{\mathcal{F}}^{*}(A)=\mathcal{H o m} \operatorname{Mod}_{A}^{\mathcal{F}}(A, A)
$$

to be the object of $\mathcal{M}$ representing the endomorphisms of $A$ as an object of $\operatorname{Mod}_{A}^{\mathcal{F}}(\mathcal{M})$.

In particular, when $\mathcal{M}=\mathcal{P} r^{\mathrm{L}}$, the $\mathcal{F}$-Hochschild cohomology of an $\mathcal{F}$-category $\mathcal{C}$ is the $\infty$-category of $\mathcal{F}$ - $\mathcal{C}$-module functors

$$
\operatorname{HH}_{\mathcal{F}}^{*}(\mathcal{C})=\operatorname{Fun}_{\operatorname{Mod}_{\mathcal{C}}^{\mathcal{I}}}(\mathcal{C}, \mathcal{C}) .
$$


We now specialize to the case where $\mathcal{F}$ is the $\mathcal{E}_{n}$-operad. Intuitively, an $\mathcal{E}_{n^{-}}$ algebra structure on an object $A$ is equivalent to a family of associative algebra structures on $A$ parameterized by $S^{n-1}$ such that antipodal points are associated to opposite multiplications on $A$. An $\mathcal{E}_{n}$ - $A$-module structure on $M$ admits a similar intuitive interpretation as a family of compatible left $A$-module structures on $M$ parameterized by $S^{n-1}$. This intuition leads to the following (to appear in [F2]; see also [L7]).

Proposition 5.9 ([F2]). Let $\mathcal{C}$ be a presentable symmetric monoidal $\infty$-category whose monoidal structure distributes over colimits.

To an $\mathcal{F}$-algebra $A$ in $\mathcal{C}$ there is a functorially assigned associative algebra $U_{A}$ such that there is a canonical equivalence $\operatorname{Mod}_{A}^{\mathcal{F}}(\mathcal{C}) \simeq \operatorname{Mod}_{U_{A}}(\mathcal{C})$ between $\mathcal{F}-A$ modules and left $U_{A}$-modules.

If $\mathcal{F}$ is the $\mathcal{E}_{n}$-operad, and the $\mathcal{E}_{n}$-algebra structure on $A$ is obtained by restriction from an $\mathcal{E}_{\infty}$-algebra structure, then there is a canonical equivalence of associative algebras $U_{A} \simeq S^{n-1} \otimes A$.

For $n=1$, the proposition reduces to the familiar statement that for an $\mathcal{E}_{\infty}$ algebra $A$, an $A \otimes A^{\mathrm{op}}$-module structure (in the form of an $\mathcal{E}_{1}$ - $A$-module structure) is equivalent to an $A \otimes A$-module structure (in the form of a left $U_{A}$-module structure).

For our current purposes, one can interpret the proposition as furnishing the definition of an $\mathcal{E}_{n}-A$-module. Namely, the reader uncomfortable with the abstractions can take left $S^{n-1} \otimes A$-modules as the definition of $\mathcal{E}_{n}$ - $A$-modules

Example 5.10. Let $\mathcal{C}$ be an $\mathcal{E}_{1}$-algebra in $\mathcal{P} r^{\mathrm{L}}$ (so $\mathcal{C}$ is a presentable monoidal $\infty$ category whose monoidal structure distributes over colimits). Then left modules for the monoidal $\infty$-category $U_{\mathcal{C}}$ are equivalent to $\mathcal{C}$-bimodules. In particular, we have an equivalence $U_{\mathcal{C}} \simeq \mathcal{C} \otimes \mathcal{C}^{\text {op }}$, where here $\mathcal{C}^{\text {op }}$ denotes the $\infty$-category $\mathcal{C}$ equipped with the opposite monoidal structure. As a consequence, we see that in this case, the preceding general definition of $\mathcal{F}$-Hochschild cohomology recaptures the notion of the Drinfeld center introduced earlier.

Let $A$ be an $\mathcal{E}_{\infty}$-algebra and $U_{A}$ be as above. We have a companion definition of $\mathcal{E}_{n}$-Hochschild homology.

Definition 5.11. For an $\mathcal{E}_{\infty}$-algebra $A$, we define the $\mathcal{E}_{n}$-Hochschild homology

$$
\mathrm{HH}_{*}^{\mathcal{E}_{n}}(A)=A \otimes_{U_{A}} A
$$

to be the tensor product of $A$ with itself over the algebra $U_{A}$.

We now have the following contribution of this paper to the story.

Corollary 5.12. For a perfect stack $X$, consider the stable $\infty$-category $\mathrm{QC}(X)$ equipped with its $\mathcal{E}_{n}$-tensor product. Then with $X^{S^{n}}=\operatorname{Map}\left(S^{n}, X\right)$, there are canonical equivalences

$$
\mathrm{QC}\left(X^{S^{n}}\right) \simeq \mathrm{HH}_{\mathcal{E}_{n}}^{*}(\mathrm{QC}(X)) \simeq \mathrm{HH}_{*}^{\mathcal{E}_{n}}(\mathrm{QC}(X)) .
$$

Proof. The result follows from an inductive application of Theorem 4.7 and Corollary 4.12 to the Cartesian diagrams

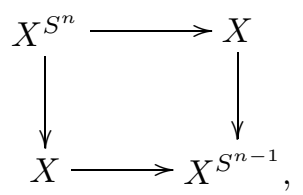


where the two maps $X \rightarrow X^{S^{n-1}}$ assign to a point of $X$ the corresponding constant map.

\section{Epilogue: Topological Field THEORY}

For a perfect stack $X$, the $\infty$-category $\mathrm{QC}(\mathcal{L} X)$ of sheaves on its loop space carries a rich structure coming from topological field theory, generalizing the braided tensor category structure on the usual Drinfeld center and providing a categorified analogue of the Deligne conjecture on Hochschild cochains. In this section, we discuss these structures and their generalizations.

6.1. Topological field theory from perfect stacks. Let 2 Cob denote the $\infty$ category whose objects are compact oriented 1-manifolds, and whose morphisms $2 \operatorname{Cob}\left(C_{1}, C_{2}\right)$ consist of the classifying spaces of oriented topological surfaces with fixed incoming and outgoing components $C_{1}, C_{2}$. This $\infty$-category has a symmetric monoidal structure given by the disjoint union of 1-manifolds. We will abuse notation by denoting the object consisting of the disjoint union of $m$ copies of $S^{1}$ by $m$.

Definition 6.1. A 2-dimensional topological field theory valued in a symmetric monoidal $\infty$-category $\mathcal{C}$ is a symmetric monoidal functor $F: 2 \mathrm{Cob} \rightarrow \mathcal{C}$.

Fix a base commutative derived ring $k$.

Definition 6.2. Let Cat ex $_{\infty}^{\mathrm{x}}$ denote the $(\infty, 2)$-category of stable, presentable $k$ linear $\infty$-categories, with 1-morphisms consisting of continuous exact functors.

Note that $\mathrm{Cat}_{\infty}^{\mathrm{ex}}$ has a symmetric monoidal structure, the tensor product of presentable stable categories as studied in [L4, 4.2], and the unit of the monoidal structure is the $\infty$-category of $k$-modules. Since the empty 1 -manifold is the unit of $2 \mathrm{Cob}$, a topological field theory $\mathcal{Z}$ sends its endomorphisms, which are closed surfaces $\Sigma$, to endomorphisms of the unit of the target $\infty$-category $\mathcal{C}$. When $\mathcal{C}=$ $\mathrm{Cat}_{\infty}^{\mathrm{ex}}$, the unit is $\operatorname{Mod}_{k}$ and $\mathcal{Z}(\Sigma)$ is a $k$-module.

Proposition 6.3. For a perfect stack $X$, there is a $2 d$ TFT $\mathcal{Z}_{X}: 2 \mathrm{Cob} \rightarrow \mathrm{Cat}_{\infty}^{\mathrm{ex}}$ with $\mathcal{Z}_{X}\left(S^{1}\right)=\mathrm{QC}(\mathcal{L} X)$ and $\mathcal{Z}_{X}(\Sigma)=\Gamma\left(X^{\Sigma}, \mathcal{O}_{X^{\Sigma}}\right)$, for closed surfaces $\Sigma$.

Proof. We define $\mathcal{Z}_{X}$ on objects by assigning $\mathcal{Z}_{X}(m)=\mathrm{QC}\left((\mathcal{L} X)^{m}\right)$.

To define $\mathcal{Z}_{X}$ on morphisms, observe first that since $X$ is perfect, $(\mathcal{L} X)^{m}$ is perfect, and for $\Sigma \in 2 \operatorname{Cob}^{\circ}(m, n)$, the mapping stack $X^{\Sigma}=\operatorname{Map}(\Sigma, X)$ is also perfect (special cases of Corollary 3.25). Now for each $\Sigma \in 2 \mathrm{Cob}^{\circ}(m, n)$, consider the correspondence

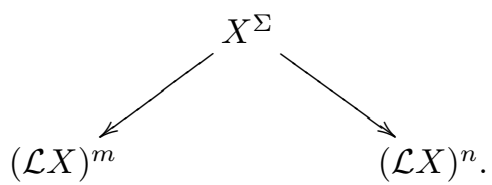

Since all of the stacks involved are perfect, pullback and pushforward of quasicoherent sheaves along this correspondence defines a colimit-preserving functor

$$
\mathcal{Z}_{X}(\Sigma): \mathrm{QC}\left((\mathcal{L} X)^{m}\right) \rightarrow \mathrm{QC}\left((\mathcal{L} X)^{n}\right)
$$

Thus applying this construction in families, we obtain a map of spaces

$$
\mathcal{Z}_{X}(m, n): 2 \operatorname{Cob}^{\circ}(m, n) \rightarrow \operatorname{Fun}\left(\mathrm{QC}\left((\mathcal{L} X)^{m}\right), \mathrm{QC}\left((\mathcal{L} X)^{n}\right)\right) .
$$


Suppose now that $\Sigma=\Sigma_{1} \coprod_{\coprod_{k} S^{1}} \Sigma_{2} \in 2 \operatorname{Cob}(m, n)$ is obtained by sewing two surfaces $\Sigma_{1} \in 2 \operatorname{Cob}(m, k)$ and $\Sigma_{2} \in 2 \operatorname{Cob}(k, n)$. Then we have a diagram of correspondences

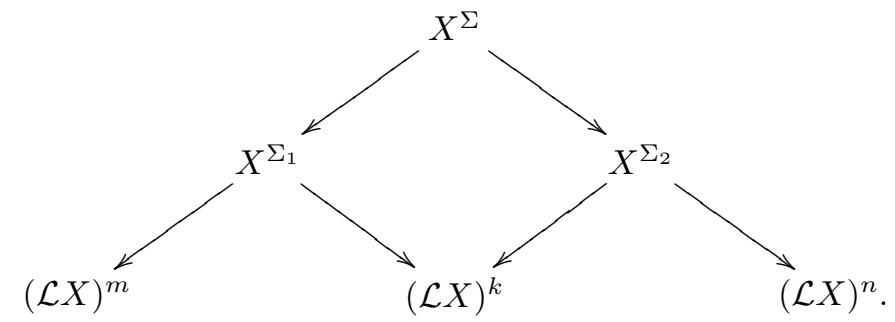

Since all of the stacks involved are perfect, base change provides a canonical equivalence of functors

$$
\mathcal{Z}_{X}(\Sigma) \simeq \mathcal{Z}_{X}\left(\Sigma_{2}\right) \circ \mathcal{Z}_{X}\left(\Sigma_{1}\right): \mathrm{QC}\left((\mathcal{L} X)^{m}\right) \rightarrow \mathrm{QC}\left((\mathcal{L} X)^{n}\right) .
$$

Similar diagrams define the higher compositions.

To complete the construction, note that $\mathcal{Z}_{X}$ comes equipped with a canonical symmetric monoidal structure. Namely, by Theorem 4.7 there is a canonical equivalence

$$
\mathcal{Z}_{X}(m)=\mathrm{QC}\left((\mathcal{L} X)^{m}\right) \simeq \mathrm{QC}(\mathcal{L} X)^{\otimes m}=\mathcal{Z}_{X}(1)^{\otimes m},
$$

and it clearly extends to a symmetric monoidal structure.

As an example, take $X=B G$ (in characteristic zero). Then $\mathcal{Z}_{B G}\left(S^{1}\right)$ is the $\infty$-category of sheaves on the adjoint quotient $G / G=\operatorname{Loc}_{G}\left(S^{1}\right)$, while $\mathcal{Z}_{B G}(\Sigma)$ is the cohomology of the structure sheaf of the moduli stack $B G^{\Sigma}=\operatorname{Loc}_{G}(\Sigma)$ of $G$-local systems on $\Sigma$.

More generally, the particular topological field theory operations we are considering are not sensitive to the structure of manifolds, and the same construction provides a field theory living over "bordisms" of spaces. In what follows, by a space we will mean a space homotopy equivalent to a finite simplicial set.

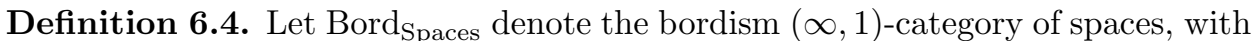
1-morphisms from $U$ to $V$ given by spaces $T_{U V}$ with maps $U \rightarrow T_{U V} \leftarrow V$, and composition of $T: U \rightarrow V$ and $T^{\prime}: V \rightarrow W$ given by the homotopy pushout of spaces

$$
T^{\prime} \circ T=T \amalg_{V} T^{\prime} .
$$

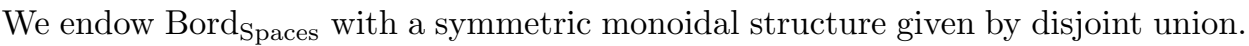

The proof of the previous proposition immediately extends to give the following:

Proposition 6.5. For any perfect stack $X$, there is a symmetric monoidal functor

$$
S_{X}: \text { Bord }_{\text {Spaces }} \rightarrow \mathrm{Cat}_{\infty}^{\mathrm{ex}}
$$

with $S_{X}(U)=\mathrm{QC}\left(X^{U}\right)$ and $S_{X}(T: U \rightarrow V)$ the functor $\mathrm{QC}(U) \rightarrow \mathrm{QC}(V)$ given by pullback and pushforward along the correspondence

$$
X^{U} \leftarrow X^{T} \rightarrow X^{V} \text {. }
$$


The $2 \mathrm{~d}$ topological field theory $\mathcal{Z}_{X}$ above is a categorified analogue of the $2 \mathrm{~d}$ TFTs defined by string topology on a compact oriented manifold, or the topological B-model defined by a Calabi-Yau variety [C]. Namely, we assign to the circle the $\infty$-category of quasi-coherent sheaves, rather than the complex of chains, on the loop space. This has the advantage that we may construct maps for arbitrary correspondences without the assumption that $X$ is a Calabi-Yau variety or satisfies Poincaré duality. On the other hand, we have gone up a level of categoricity, pushing off the problems of duality and orientation to the definition of operations between $\Gamma\left(X^{\Sigma}, \mathcal{O}_{X^{\Sigma}}\right)$ for cobordisms between surfaces or invariants for 3-manifolds. Recall BK] that an extended 3-dimensional topological field theory assigns a braided (in fact ribbon) category to the circle. This category must however satisfy a strong finiteness and nondegeneracy condition (modularity) coming from its extension to three-manifolds. It would be interesting to see what conditions need to be imposed on the sheaves we consider and on the stack $X$ in order to extend $\mathcal{Z}_{X}$ in such a fashion. Note that Corollary 4.8 shows that $\mathrm{QC}(X)$ for $X$ perfect satisfies an analogue of the Calabi-Yau or Frobenius property: namely it is self-dual as a $\operatorname{Mod}_{k^{-}}$ module.

6.2. Deligne-Kontsevich conjectures for derived centers. The notion of Drinfeld center for monoidal stable categories is a categorical analogue of Hochschild cohomology of associative (or $A_{\infty}$ ) algebras (or more precisely of Hochschild cochains, or its spectral analogue, topological Hochschild cohomology). In the case of algebras, Deligne's conjecture states that the Hochschild cochain complex has the structure of an $\mathcal{E}_{2}$-algebra (lifting the Gerstenhaber algebra, or $H_{*}\left(\mathcal{E}_{2}\right)$-algebra, structure on Hochschild cohomology). There is also a cyclic version of the conjecture which states that the Hochschild cochains for a Frobenius algebra possesses the further structure of a framed $\mathcal{E}_{2}$, or ribbon, algebra. See [T1, K1, KS1, MS, LC, KS2 for various proofs of the Deligne conjecture and [Kau, TZ for its cyclic version. The Kontsevich conjecture (see [T2, HKV]) generalizes this picture to higher algebras, asserting that the Hochschild cochains on an $\mathcal{E}_{n}$-algebra have a natural $\mathcal{E}_{n+1}$-structure.

The work of Costello [C] and Kontsevich-Soibelman [KS2 explains how a strong form of the Deligne conjecture follows from the structure of topological field theory, specifically, by constructing a topological field theory $\mathcal{Z}_{A}$ from an algebra $A$ such that $\mathcal{Z}_{A}\left(S^{1}\right)$ is the Hochschild cochain complex of $A$. Observe that the configuration space of $m$-tuples of circles in the disk (the $m$-th space of the $\mathcal{E}_{2}$-operad) maps to the space of functors $\mathcal{Z}\left(S^{1}\right)^{\otimes m} \rightarrow \mathcal{Z}\left(S^{1}\right)$ compatibly with compositions.

Proposition 6.6. In any $2 d T F T \mathcal{Z}$, the object $\mathcal{Z}\left(S^{1}\right) \in \mathcal{C}$ is an algebra over the framed $\mathcal{E}_{2}$-operad (in particular, over the $\mathcal{E}_{2}$-operad).

A natural categorified analogue of the Deligne conjecture states that the Drinfeld center of a monoidal $\infty$-category is an $\mathcal{E}_{2}$-category. For dualizable and self-dual $k$ linear $\infty$-categories, one can ask if this structure comes from a natural framed $\mathcal{E}_{2}$, or ribbon, structure. We observe that our identification of the Drinfeld center of $\mathrm{QC}(X)$ with sheaves on the loop space, combined with the construction of the topological field theory $\mathcal{Z}_{X}$, automatically solves this conjecture in the applicable 
cases:

Corollary 6.7. For $X$ a perfect stack, the center $\mathcal{T} r(\mathrm{QC}(X)) \simeq \mathrm{QC}(\mathcal{L} X)$ has the structure of a stable framed $\mathcal{E}_{2}$-category. For a map $X \rightarrow Y$ of perfect stacks, the same holds for the center $\operatorname{Tr}\left(\mathrm{QC}\left(X \times_{Y} X\right)\right) \simeq \mathrm{QC}(\mathcal{L} X)$.

In fact, the same arguments prove a case of the categorified form of the Kontsevich conjecture. We consider $\mathrm{QC}\left(X^{S^{n}}\right)$ as part of an $(n+1)$-dimensional topological field theory, with operations given by cobordisms of $n$-manifolds.

Corollary 6.8. For $X$ a perfect stack, the $\mathcal{E}_{n}$-Hochschild cohomology of the stable $\mathcal{E}_{n}$-category $\mathrm{QC}(X)$ has the structure of a stable (framed) $\mathcal{E}_{n+1}$-category.

\section{ACKNOWLEDGEMENTS}

This paper relies heavily on the work of Jacob Lurie, whom the authors thank for many helpful discussions and for his generosity in sharing his knowledge (and in particular for the suggestion of how to describe the higher $\mathcal{E}_{n}$-centers of $\mathrm{QC}(X)$ geometrically). The first and last authors would like to thank Bertrand Toën for educating them in derived algebraic geometry and patiently explaining many arguments. They would also like to thank Victor Ostrik for sharing his understanding of Drinfeld centers and their applications to Hecke categories. The second author thanks his advisor, Michael Hopkins, for discussions on the topological field theory aspects of this paper, as well as for his support and guidance. Many thanks to Andrew Blumberg and Amnon Neeman for detailed comments on early drafts. Finally, we would also like to thank the anonymous referees for their valuable feedback.

The first author is partially supported by NSF CAREER grant DMS-0449830. The second author was supported by an NSF Graduate Research Fellowship. The last author is partially supported by NSF grant DMS-0600909 and a Sloan Research Fellowship. Work on this paper has taken place at the Aspen Center for Physics and the IAS (supported by NSF grant DMS-0635607), whom the authors thank for providing support and stimulating environments.

\section{REFERENCES}

[A] V. Angeltveit, The cyclic bar construction on $A_{\infty} H$-spaces. Adv. Math. 222 (2009), 1589-1610. arXiv:math/0612165. MR2555906

[BK] B. Bakalov and A. Kirillov, Jr., Lectures on tensor categories and modular functors. University Lecture Series, 21. American Mathematical Society, Providence, RI, 2001. MR.1797619 (2002d:18003)

[BN1] D. Ben-Zvi and D. Nadler, Loop Spaces and Connections. arXiv:1002.3636.

[BN2] D. Ben-Zvi and D. Nadler, The character theory of a complex group. arXiv:0904.1247.

[Ber] J. Bergner, A survey of $(\infty, 1)$-categories. arXiv:math/0610239.

[Be] R. Bezrukavnikov, Noncommutative counterparts of the Springer resolution. International Congress of Mathematicians. Vol. II, 1119-1144, Eur. Math. Soc., Zürich, 2006. arXiv:math.RT/0604445. MR2275638(2009d:17026)

$[\mathrm{BeF}] \quad$ R. Bezrukavnikov and M. Finkelberg, Equivariant Satake category and KostantWhittaker reduction. Moscow Math J. 8 (2008), no. 1, 39-72, 183. arXiv:0707.3799. MR2422266 (2009d:19008)

[Bo] M. Bökstedt, Topological Hochschild Homology. Preprint, 1985.

[BoN] M. Bökstedt and A. Neeman, Homotopy limits in triangulated categories. Compositio Math. 86 (1993), no. 2, 209-234. MR1214458 (94f:18008)

[BLL] A. Bondal, M. Larsen and V. Lunts, Grothendieck ring of pretriangulated categories. Int. Math. Res. Not. 2004, no. 29, 1461-1495. arXiv:math/0401009. MR2051435 (2005d:18014) 
[BV] A. Bondal and M. Van den Bergh, Generators and representability of functors in commutative and noncommutative geometry. Mosc. Math. J. 3 (2003), no. 1, 1-36, 258. MR1996800(2004h:18009)

[Ch] C. Chevalley, Théorie des groupes de Lie. Tome II. Groupes algébriques. Actualités Sci. Ind. no. 1152. Hermann \& Cie., Paris, 1951. MR0051242 (14:448d)

[C] K. Costello, Topological conformal field theories and Calabi-Yau categories. Adv. Math. 210 (2007), no. 1, 165-214. arXiv:math/0412149. MR2298823 (2008f:14071)

[De] P. Deligne, Catégories tannakiennes. The Grothendieck Festschrift, Vol. II, 111-195, Progr. Math., 87, Birkhäuser Boston, Boston, MA, 1990. MR.1106898 (92d:14002)

[D] V. Drinfeld, DG quotients of DG categories. J. Algebra 272 (2004), no. 2, 643-691. MR2028075 (2006e:18018)

[EKMM] A. Elmendorf, I. Kriz, M. Mandell and J.P. May, Rings, modules, and algebras in stable homotopy theory. With an appendix by M. Cole. Mathematical Surveys and Monographs, 47. American Mathematical Society, Providence, RI, 1997. MR1417719 (97h:55006)

[F1] J. Francis, Derived algebraic geometry over $\mathcal{E}_{n}$-rings. MIT Ph.D. thesis, 2008.

[F2] J. Francis, The cotangent complex and Hochschild homology of $\mathcal{E}_{n}$-rings. In preparation.

[Fr] D. Freed, Higher algebraic structures and quantization. Comm. Math. Phys. 159 (1994) 343-398. MR.1256993 (95c:58034)

[H] V. Hinich, Drinfeld double for orbifolds. Quantum groups, 251-265, Contemp. Math., 433, Amer. Math. Soc., Providence, RI, 2007. arXiv:math.QA/0511476. MR 2349625 (2009a:14002)

[HS] A. Hirschowitz and C. Simpson, Descente pour les $n$-champs. arXiv:math/9807049.

[HPS] M. Hovey, J. Palmieri and N. Strickland, Axiomatic stable homotopy theory. Mem. Amer. Math. Soc. 128 (1997), no. 610. MR.1388895 (98a:55017)

[HSS] M. Hovey, B. Shipley and S. Schwede, Symmetric Spectra. J. Amer. Math. Soc. 13 (2000), no. 1, 149-208. arXiv:math/9801077. MR1695653 (2000h:55016)

[HKV] P. Hu, I. Kriz and A. Voronov, On Kontsevich's Hochschild cohomology conjecture. Compos. Math. 142 (2006), no. 1, 143-168. MR2197407 (2006j:18003)

[Jo] A. Joyal, Quasi-categories and Kan complexes. J. Pure Appl. Algebra 175 (2002) 207222. MR.1935979 (2003h:55026)

[JS] A. Joyal and R. Street, Tortile Yang-Baxter operators in tensor categories. J. Pure Appl. Algebra 71 (1991), no. 1, 43-51. MR.1107651 (92e:18006)

[Kap] M. Kapranov, Rozansky-Witten invariants via Atiyah classes. Compositio Math. 115 (1999), no. 1, 71-113. math.AG/9704009. MR1671737(2000h:57056)

[KRS] A. Kapustin, L. Rozansky and N. Saulina, Three-dimensional topological field theory and symplectic algebraic geometry I. Nuclear Phys. B 816 (2009), no. 3, 295-355. arXiv:0810.5415. MR 2522724

[Kau R. Kaufmann, A proof of a cyclic version of Deligne's conjecture via cacti. Math. Res. Lett. 15 (2008), no. 5, 901-921. math.QA/0403340. MR2443991(2009i:18006)

[Ke] B. Keller, On differential graded categories. International Congress of Mathematicians. Vol. II, 151-190, Eur. Math. Soc., Zürich, 2006. arXiv:math.AG/0601185. MR2275593 (2008g:18015)

[K1] M. Kontsevich, Operads and motives in deformation quantization. Moshé Flato (19371998). Lett. Math. Phys. 48 (1999), no. 1, 35-72. MR1718044 (2000j:53119)

[K2] M. Kontsevich, Rozansky-Witten theory via formal geometry. Compositio Math. 115 (1999), no. 1, 115-127. arXiv:dg-ga/9704009. MR1671725(2000h:57057)

[KS1] M. Kontsevich and Y. Soibelman, Deformations of algebras over operads and the Deligne conjecture.Conference Moshé Flato 1999, Vol. I (Dijon), 255-307, Math. Phys. Stud., 21, Kluwer Acad. Publ., Dordrecht, 2000. MR.1805894 (2002e:18012)

[KS2] M. Kontsevich and Y. Soibelman, Notes on A-infinity algebras, A-infinity categories and non-commutative geometry I. Preprint arXiv:math/0606241.

[LMS] L.G. Lewis, Jr., J.P. May, and M. Steinberger, Equivariant stable homotopy theory. With contributions by J. McClure. Lecture Notes in Mathematics, 1213. Springer-Verlag, Berlin, 1986. MR866482(88e:55002)

[L1] J. Lurie, Derived Algebraic Geometry, MIT Ph.D. Thesis, 2004

[L2] J. Lurie, Higher topos theory. Annals of Mathematics Studies, 170. Princeton University Press, 2009. arXiv:math.CT/0608040. MR2522659 
[L3] J. Lurie, Derived Algebraic Geometry 1: Stable infinity categories. arXiv:math.CT/ 0608228.

[L4] J. Lurie, Derived Algebraic Geometry 2: Noncommutative algebra. arXiv:math.CT/ 0702299

[L5] J. Lurie, Derived Algebraic Geometry 3: Commutative algebra. arXiv:math.CT/ 0703204 .

[L6] J. Lurie, On the Classification of Topological Field Theories. Current developments in mathematics, 2008, pp. 129-280, Int. Press, Somerville, MA, 2009. arXiv:0905.0465. MR2555928

[L7] J. Lurie, Derived Algebraic Geometry 6: $E_{k}$ Algebras. arXiv:0911.0018.

[MS] J. McClure and J. Smith, A solution of Deligne's Hochschild cohomology conjecture. Recent progress in homotopy theory (Baltimore, MD, 2000), 153-193, Contemp. Math., 293, Amer. Math. Soc., Providence, RI, 2002. MR1890736 (2003f:55013)

[M] M. Müger, From subfactors to categories and topology II. The quantum double of tensor categories and subfactors. J. Pure Appl. Algebra 180 (2003), no. 1-2, 159-219. arXiv:math.CT/0111205. MR1966525 (2004f:18014)

[N1] A. Neeman, The connection between the $K$-theory localization theorem of Thomason, Trobaugh and Yao and the smashing subcategories of Bousfield and Ravenel. Ann. Sci. École Norm. Sup. (4) 25 (1992), no. 5, 547-566. MR.1191736 (93k:18015)

[N2] A. Neeman, The Grothendieck duality theorem via Bousfield's techniques and Brown representability. J. Amer. Math. Soc. 9 (1996), no. 1, 205-236. MR.1308405 (96c:18006)

[N3] A. Neeman, Triangulated categories. Annals of Mathematics Studies, 148. Princeton University Press, Princeton, NJ, 2001. MR.1812507 (2001k:18010)

[O] D. Orlov, Equivalences of derived categories and $K 3$ surfaces. Algebraic geometry, 7. J. Math. Sci. (New York) 84 (1997), no.5, 1361-1381. arXiv:math.AG/9606006. MR.1465519(99a:14054)

[Os] V. Ostrik, Module categories over the Drinfeld double of a finite group. Int. Math. Res. Not. 2003, no. 27, 1507-1520. arXiv:math.QA/0202130. MR.1976233 (2004h:18005)

[RobW] J. Roberts and S. Willerton, On the Rozansky-Witten weight systems. math.AG/ 0602653

[RW] L. Rozansky and E. Witten, Hyper-Kähler geometry and invariants of three-manifolds. Selecta Math. (N.S.) 3 (1997), no. 3, 401-458. MR.1481135 (98m:57041)

[SSh] S. Schwede and B. Shipley, Stable model categories are categories of modules. Topology 42 (2003), no. 1, 103-153. MR.1928647 (2003g:55034)

[Sh] B. Shipley, $H \mathbb{Z}$-algebra spectra are differential graded algebras. Amer. J. Math. 129 (2007), no. 2, 351-379. MR.2306038 (2008b:55015)

[T1] D. Tamarkin, Another proof of M. Kontsevich formality theorem. arXiv:math/9803025.

[T2] D. Tamarkin, The deformation complex of a $d$-algebra is a $(d+1)$-algebra. arXiv:math/ 0010072 .

[TT] R. Thomason and T. Trobaugh, Higher algebraic $K$-theory of schemes and of derived categories. The Grothendieck Festschrift, Vol. III, 247-435, Progr. Math., 88, Birkhäuser Boston, Boston, MA, 1990. MR 1106918 (92f:19001)

[To1] B. Toën, The homotopy theory of dg categories and derived Morita theory. Invent. Math. 167 (2007), no. 3, 615-667. arXiv:math.AG/0408337. MR2276263 (2008a:18006)

[To2] B. Toën, Higher and Derived Stacks: a global overview. Proceedings 2005 AMS Summer School in Algebraic Geometry. Proc. Sympos. Pure Math., Vol. 80, Part 1, Amer. Math. Soc., Providence, RI, 2009. arXiv:math.AG/0604504. MR 2483943

[ToVe1] B. Toën and G. Vezzosi, Homotopical Algebraic Geometry I: Topos theory. Adv. Math. 193 (2005), no. 2, 257-372. arXiv:math.AG/0404373. MR/2137288 (2007b:14038)

[ToVe2] B. Toën and G. Vezzosi, Homotopical Algebraic Geometry II: Geometric stacks and applications. Memoirs of the AMS 193 (2008). arXiv:math.AG/0404373. MR 2394633 (2009h:14004)

[TZ] T. Tradler and M. Zeinalian, On the cyclic Deligne conjecture. J. Pure Appl. Algebra 204 (2006), no. 2, 280-299. math.QA:0404218. MR2184812 (2007j:16015) 
Department of Mathematics, University of Texas, Austin, Texas 78712-0257

E-mail address: benzvi@math.utexas.edu

Department of Mathematics, Northwestern University, Evanston, Illinois 60208-2370

E-mail address: jnkf@math.northwestern.edu

Department of Mathematics, Northwestern University, Evanston, Illinois 60208-2370

E-mail address: nadler@math.northwestern.edu 